TRANSACTIONS OF THE

AMERICAN MATHEMATICAL SOCIETY

Volume 362, Number 7, July 2010, Pages 3391-3434

S 0002-9947(10)04902-0

Article electronically published on February 17, 2010

\title{
A LIOUVILLE THEOREM \\ FOR VECTOR VALUED SEMILINEAR HEAT EQUATIONS WITH NO GRADIENT STRUCTURE AND APPLICATIONS TO BLOW-UP
}

\author{
NEJLA NOUAILI AND HATEM ZAAG
}

\begin{abstract}
We prove a Liouville theorem for a vector valued semilinear heat equation with no gradient structure. Classical tools such as the maximum principle or energy techniques break down and have to be replaced by a new approach. We then derive from this theorem uniform estimates for blow-up solutions of that equation.
\end{abstract}

\section{INTRODUCTION}

This paper is concerned with blow-up solutions of the semilinear heat equation

$$
\partial_{t} u=\Delta u+F(u),
$$

where $u(t): x \in \mathbb{R}^{N} \rightarrow \mathbb{R}^{M}, \Delta$ denotes the Laplacian and $F: \mathbb{R}^{M} \rightarrow \mathbb{R}^{M}$ is not necessarily a gradient. We say that $u(t)$ blows up in finite time $T$ if $u(t)$ exists for all $t \in[0, T)$ and

$$
\lim _{t \rightarrow T}\|u(t)\|_{L^{\infty}}=+\infty .
$$

We note that an extensive literature is devoted to the study of equation (1.1). Many results were found using monotonicity properties, the maximal principle (valid for scalar equations) or energy techniques (valid when $F$ is a gradient). See for example Wei84, Fuj66], Bal77], Lev73. Unfortunately, there are important classes of PDEs where these techniques break down. For example, equations of the type (1.1), where $F$ is not a gradient, or PDEs coming from geometric flows; see for example a review paper by Hamilton Ham95.

In this work, we would like to develop new tools for a class of equations where classical tools do not work, in particular, vector valued equations with no gradient structure. More precisely, we will consider the following reaction-diffusion equation:

$$
u_{t}=\Delta u+(1+i \delta)|u|^{p-1} u, u(0, x)=u_{0}(x),
$$

Received by the editors October 24, 2007.

2000 Mathematics Subject Classification. Primary 35B05, 35K05, 35K55, 74H35.

Key words and phrases. Blow-up, Liouville theorem, uniform estimates, heat equation, vector valued.

The authors would like to thank the referee for his valuable suggestions which (we hope) made our paper much clearer and reader friendly.

The second author was supported by a grant from the French Agence Nationale de la Recherche, project ONDENONLIN, reference ANR-06-BLAN-0185. 
where $u(t): \mathbb{R}^{N} \rightarrow \mathbb{C}, \delta \in \mathbb{R}$ and

$$
p>1 \text { and }(N-2) p<(N+2) .
$$

Note that the nonlinearity in this equation is not a gradient. Note also that (1.2) is a particular case of the complex Ginzburg-Landau equation

$$
\partial_{t} u=(1+i \beta) \Delta u+(\epsilon+i \delta)|u|^{p-1} u-\gamma u, \text { where }(x, t) \in \mathbb{R}^{N} \times(0, T),
$$

$\beta, \delta$ and $\gamma$ real, $p>1$ and $\epsilon= \pm 1$.

This equation is mostly famous when $\epsilon=-1$. It appears in the study of various physical problems (plasma physics, nonlinear optics). It is in particular used as an amplitude equation near the onset of instabilities in fluid mechanics (see for example Levermore and Oliver [LO96]). In this case, Plecháč and Šverák PŠ01] used matching techniques and numerical simulations to give strong evidence for the existence of blow-up solutions in the focusing case, namely $\beta \delta>0$.

The case $\varepsilon=1$ is less famous. To our knowledge, there is only the work of Popp, Stiller, Kuznetsov and Kramer [PKK98, who use a formal approach to find blow-up solutions. More recently, Masmoudi and Zaag [MZ08a gave a constructive method to show the existence of a stable blow-up solution under some conditions for the parameters.

Let us present in the following the known results for equation (1.2) and most importantly the research directions and open problems. In the study of the blow-up phenomenon for equation (1.2), we believe that there are two important issues:

Construction of examples of blow-up solutions: In this approach, one has to construct examples of solutions that blow up in finite time. In particular, one has to find conditions on initial data and/or parameters of the equation to guarantee that the solution blows up in finite time. For equation (1.2), we recall the result obtained by Zaag [Zaa98] (the range of $\delta$ has been widened in [MZ08a]):

For each $\delta \in(-\sqrt{p}, \sqrt{p})$,

i) equation (1.2) has a solution $u(x, t)$ on $\mathbb{R}^{N} \times[0, T)$ which blows up in finite time $T>0$ at only one blow-up point $a \in \mathbb{R}^{N}$,

ii) moreover, we have

$$
\lim _{t \rightarrow T}\left\|(T-t)^{\frac{1+i \delta}{p-1}} u\left(a+((T-t)|\log (T-t)|)^{\frac{1}{2}} z, t\right)-f_{\delta}(z)\right\|_{L^{\infty}\left(\mathbb{R}^{N}\right)}=0
$$

with

$$
f_{\delta}(z)=\left(p-1+\frac{(p-1)^{2}}{4\left(p-\delta^{2}\right)}|z|^{2}\right)^{-\frac{1+i \delta}{p-1}},
$$

iii) there exists $u_{*} \in \mathcal{C}\left(\mathbb{R}^{N} \backslash\{a\}, \mathbb{C}\right)$ such that $u(x, t) \rightarrow u_{*}(x)$ as $t \rightarrow T$ uniformly on compact subsets of $\mathbb{R}^{N} \backslash\{a\}$ and

$$
u_{*}(x) \sim\left[\frac{8\left(p-\delta^{2}\right)|\log | x-a||}{(p-1)^{2}|x-a|^{2}}\right]^{\frac{1+i \delta}{p-1}} \text { as } x \rightarrow a .
$$

Remark 1.1. In [MZ08a], the same result was proved for equation (1.4), where the linearized operator around the expected profile is much more difficult to study.

Asymptotic behavior for any arbitrary blow-up solution: In this approach, one takes any arbitrary blow-up solution for equation (1.2) and tries to describe its blow-up behavior. More precisely, it consists in the determination of the asymptotic profile (that is, a function from which, after a time-dependent scaling, $u(t)$ approaches as $t \rightarrow T$ ) of the blow-up solution. 
In earlier literature, the determination of the profile is done through the study of entire solutions (defined for all time and space) of the equation. See for example Grayson and Hamilton [GH96] for the case of the harmonic map heat flow and Giga and Kohn GK85 for the heat equation (that is, $\delta=0$ in (1.2); there, the authors prove a Liouville theorem which turns out to be the trivial case of the Liouville theorem proved by Merle and Zaag in [MZ98a, and [MZ00] and stated in Proposition 3.4 below). Let us remark that the use of Liouville theorems was successful for elliptic equations (see Gidas and Spruck GS81a and GS81b]).

More recently, the characterization of entire solutions by means of Liouville theorems allowed us to obtain more than the blow-up profile, namely uniform estimates with respect to initial data and the singular point. See for the heat equation Merle and Zaag [MZ98b], MZ98a, [MZ00], for the modified Korteweg-de Vries equation Martel and Merle [MM00, for the nonlinear Schrödinger equation Merle and Raphael [MR04, MR05] and for the wave equation Merle and Zaag [MZ08b] and MZ08b.

The existence of a Lyapunov functional is traditionally a crucial tool in the proof of Liouville theorems, such as for the heat equation [MZ00] or the wave equation MZ08b. One wonders whether it is possible to prove a Liouville theorem for a system with no Lyapunov functional. The first attempt was done by Zaag Zaa01] for the following system:

$$
\partial_{t} u=\Delta u+v^{p}, \partial_{t} v=\Delta v+u^{q},
$$

and its self-similar version

$$
\begin{aligned}
& \partial_{s} \Phi=\Delta \Phi-\frac{1}{2} y \cdot \nabla \Phi+\Psi^{p}-\left(\frac{p+1}{p q-1}\right) \Phi \\
& \partial_{s} \Psi=\Delta \Psi-\frac{1}{2} y \cdot \nabla \Psi+\Phi^{q}-\left(\frac{q+1}{p q-1}\right) \Psi .
\end{aligned}
$$

This is the result of Zaa01:

Consider $p_{0}>1$ such that $(N-2) p_{0}<N+2$ and $M>0$. Then, there exists $\eta>0$ such that if $\left|p-p_{0}\right|+\left|q-p_{0}\right|<\eta$, then for any nonnegative $(\Phi, \Psi)$ solution of (1.7) such that for all $(y, s) \in \mathbb{R}^{N} \times \mathbb{R}, \Phi(y, s)+\Psi(y, s) \leq M$, then either $(\Phi, \Psi)=(0,0)$ or $(\Phi, \Psi)=(\Gamma, \gamma)$ or $(\Phi, \Psi)=\left(\Gamma\left(1+e^{s-s_{0}}\right)^{-\frac{p+1}{p q-1}}, \gamma\left(1+e^{s-s_{0}}\right)^{-\frac{q+1}{p q-1}}\right)$ for some $s_{0} \in \mathbb{R}$, where $(\Gamma, \gamma)$ is the only nontrivial constant solutions of (1.7) defined by

$$
\gamma^{p}=\Gamma\left(\frac{p+1}{p q-1}\right) \text { and } \Gamma^{q}=\gamma\left(\frac{q+1}{p q-1}\right)
$$

Before Zaa01, Andreucci, Herrero and Velázquez addressed the same question in AHV97 but could not determine explicitly the third case. In some sense, they just gave the limits as $s \rightarrow \pm \infty$ (for a statement, see the remark after Proposition 2.3 below). The characterization of that third case is far more difficult than the rest. The lack of a Lyapunov functional was overcome thanks to an infinite dimensional blow-up criterion.

Following system (1.6), it was interesting to address the case of equation (1.2) for $\delta \neq 0$. As for system (1.6), there is no Lyapunov functional. On the contrary, no blow-up criterion is available and the set of nonzero stationary solutions for the self-similar version is a continuum (see Proposition 2.1 below). For these two reasons, new tools have to be found, which makes our paper meaningful. 
Another reason for our work is the full Ginzburg-Landau model (1.4) with $\beta \neq 0$. That case has one more difficulty since the linearized operator in the self-similar variable becomes nonselfadjoint, as one can see from [MZ08a]. Thus, this paper is a fundamental step towards the proof of a Liouville theorem for the full GinzburgLandau model (1.4), which we believe to be an open problem of great importance.

1.1. A Liouville theorem for system (1.2). Our aim in this paper is to prove a Liouville theorem for equation (1.2). In order to do so, we introduce for each $a \in \mathbb{R}^{N}$, the following self-similar transformation:

$$
w_{a}(y, s)=(T-t)^{\frac{(1+i \delta)}{p-1}} u(x, t), y=\frac{x-a}{\sqrt{T-t}}, s=-\log (T-t) .
$$

If $u$ is a solution of (1.2), then the function $w=w_{a}$ satisfies for all $s \geq-\log T$ and $y \in \mathbb{R}^{N}$ :

$$
w_{s}=\Delta w-\frac{1}{2} y \cdot \nabla w-\frac{(1+i \delta)}{(p-1)} w+(1+i \delta)|w|^{(p-1)} w
$$

We introduce also the Hilbert space

$$
L_{\rho}^{2}=\left\{g \in L_{\text {loc }}^{2}\left(\mathbb{R}^{N}, \mathbb{C}\right), \int_{\mathbb{R}^{N}}|g|^{2} e^{-\frac{|y|^{2}}{4}} d y<+\infty\right\}, \text { where } \rho(y)=\frac{e^{-\frac{|y|^{2}}{4}}}{(4 \pi)^{N / 2}} .
$$

If $g$ depends only on the variable $y \in \mathbb{R}^{N}$, we use the notation

$$
\|g\|_{L_{\rho}^{2}}^{2}=\int_{\mathbb{R}^{N}}|g(y)|^{2} e^{-\frac{|y|^{2}}{4}} d y .
$$

If $g$ depends only on $(y, s) \in \mathbb{R}^{N} \times \mathbb{R}$, we use the notation

$$
\|g(., s)\|_{L_{\rho}^{2}}^{2}=\int_{\mathbb{R}^{N}}|g(y, s)|^{2} e^{-\frac{|y|^{2}}{4}} d y .
$$

The main result of the paper is the following Liouville theorem, which classifies certain entire solutions (i.e. solutions defined for all $(y, s) \in \mathbb{R}^{N} \times \mathbb{R}$ ) of (1.10):

Theorem 1 (A Liouville theorem for equation (1.10)). Assuming (1.3), there exist $\delta_{0}>0$ and $M:\left[-\delta_{0}, \delta_{0}\right] \rightarrow(0,+\infty]$ with $M(0)=+\infty, M(\delta) \rightarrow+\infty$ as $\delta \rightarrow 0$ and the following property:

If $|\delta| \leq \delta_{0}$ and $w \in L^{\infty}\left(\mathbb{R}^{N} \times \mathbb{R}, \mathbb{C}\right)$ is a solution of $(1.10)$ with $\|w\|_{L^{\infty}\left(\mathbb{R}^{N} \times \mathbb{R}, \mathbb{C}\right)} \leq$ $M(\delta)$, then, either $w \equiv 0$ or $w \equiv \kappa e^{i \theta_{0}}$ or $w=\varphi_{\delta}\left(s-s_{0}\right) e^{i \theta_{0}}$ for some $\theta_{0} \in \mathbb{R}$ and $s_{0} \in \mathbb{R}$, where $\varphi_{\delta}(s)=\kappa\left(1+e^{s}\right)^{-\frac{(1+i \delta)}{(p-1)}}$ and $\kappa=(p-1)^{-\frac{1}{p-1}}$.

Going back to the original variables $u(x, t)$, we rewrite this Liouville theorem as the following:

Theorem 2 (A Liouville theorem for equation (1.2)). Assuming (1.3), there exist $\delta_{0}>0$ and $M:\left[-\delta_{0}, \delta_{0}\right] \rightarrow(0,+\infty]$ with $M(0)=+\infty, M(\delta) \rightarrow+\infty$ as $\delta \rightarrow 0$ and the following property:

If $|\delta| \leq \delta_{0}$ and $u$ is a solution of (1.2) satisfying $u(x, t)(T-t)^{\frac{1}{p-1}} \in$ $L^{\infty}\left(\mathbb{R}^{N} \times(-\infty, T), \mathbb{C}\right)$ and $\left\|u(x, t)(T-t)^{\frac{1}{p-1}}\right\|_{L^{\infty}\left(\mathbb{R}^{N} \times(-\infty, T), \mathbb{C}\right)} \leq M(\delta)$, then, $u \equiv 0$ or there exists $T_{0} \geq T$ and $\theta_{0} \in \mathbb{R}$ such that for all $(x, t) \in \mathbb{R}^{N} \times(-\infty, T)$, $u(x, t)=\kappa\left(T_{0}-t\right)^{-\frac{1+i \delta}{p-1}} e^{i \theta_{0}}$. 
Remark 1.2. This result has already been proved by Merle and Zaag [MZ98a and [MZ00] (see also Nouaili [Nou08]) when $\delta=0$.

In that case, $M(0)=+\infty$, which means that any $L^{\infty}$ entire solution of (1.10), with no restriction on the size of its norm, is trivial (i.e. independent of space).

When $\delta \neq 0$, this conclusion holds only for "small" $L^{\infty}$ norms (i.e. bounded by $M(\delta)$ ). We suspect that we cannot take $M(\delta)=+\infty$. In other words, we suspect that equation (1.10) under the condition (1.3) has nontrivial solutions in $L^{\infty}$ with a high norm. We think that such solutions can be constructed in the form $w(y, s)=w_{0}(y) e^{i \omega s}$ with high $\omega$ and high $\left\|w_{0}\right\|_{L^{\infty}}$, as Popp et al. did through formal arguments on page 96 in [PKK98] when $\delta \sim \pm 3$ (which is outside our range).

Remark 1.3. Since this result was already known from MZ98a and MZ00 when $\delta=0$, the case $\delta \neq 0$ may appear as a not surprising interesting perturbation technique of the case $\delta=0$. If this is clearly true for the statement, it is certainly not the case for the method and the techniques, mainly because the gradient structure breaks down and the linearized problem is no longer selfadjoint (see the beginning of Section 2 for more details). We have to invent new tools which are far from being a simple perturbation technique. This comprises the main innovation of our work.

Remark 1.4. One may think that our result is completely standard in the context of dynamical systems. It happens that already in the case $\delta=0$, standard methods such as the center manifold theory do not apply in our case as pointed out by Filippas and Kohn in FK92, pp. 834-835. In particular, Proposition 3.8 below, whose statement is standard, does not follow from center manifold theory because the nonlinear term is not quadratic in the function space $L_{\rho}^{2}$.

1.2. Applications to type I blow-up solutions of (1.2) . As in previously cited recent blow-up literature (MZ00, MM00 and MZ08b]), Liouville theorems have important applications to blow-up for the so-called 'type I' blow-up solutions of equation (1.2), that is, solutions satisfying

$$
\forall t \in[0, T),\|u(t)\|_{L^{\infty}} \leq M(T-t)^{-\frac{1}{p-1}},
$$

where $T$ is the blow-up time. In other words, the blow-up rate is given by the associated ODE $u^{\prime}=(1+i \delta)|u|^{p-1} u$.

We know that the solution of (1.2) constructed in Zaa98 is of type $\mathbf{I}$ (and the same holds for the solution of the Ginzburg-Landau equation (1.4) constructed in [MZ08a]). However, we have been unable to prove whether all blow-up solutions of (1.2) are of type I or not. Note that when $\delta=0$, Giga and Kohn [GK85] and Giga, Matsui and Sasayama GMS04 prove that all blow-up solutions are of type I, provided that $p$ is subcritical $((N-2) p<N+2)$. When $\delta \neq 0$, the methods of [GK85] and GMS04 break down because we no longer have positivity or a Lyapunov functional.

However, following [MZ00] and Zaa01, we can derive the following estimates for type $\mathbf{I}$ blow-up solutions of (1.2):

Proposition 3 (Uniform blow-up estimates for type I solutions). Assume (1.3), consider $|\delta| \leq \delta_{0}$ and a solution $u$ of (1.2) that blows up at time $T$ and satisfies

$$
\forall t \in[0, T),\|u(t)\|_{L^{\infty}} \leq M(\delta)(T-t)^{-\frac{1}{p-1}}
$$


where $\delta_{0}$ and $M(\delta)$ are defined in Theorem 1. Then,

- (i) ( $L^{\infty}$ estimates for derivatives)

$$
\begin{array}{r}
\|u(t)\|_{L^{\infty}}(T-t)^{\frac{1}{p-1}} \rightarrow \kappa \text { and }\left\|\nabla^{k} u(t)\right\|_{L^{\infty}}(T-t)^{\frac{1}{p-1}+\frac{k}{2}} \rightarrow 0 \\
\text { as } t \rightarrow T \text { for } k=1,2 \text { or } 3 .
\end{array}
$$

- (ii) (Uniform ODE behavior) For all $\varepsilon>0$, there is $C(\varepsilon)$ such that $\forall x \in$ $\mathbb{R}^{N}, \forall t \in\left[\frac{T}{2}, T\right)$,

$$
\left.\left.\left|\frac{\partial u}{\partial t}(x, t)-(1+i \delta)\right| u\right|^{p-1} u(x, t)|\leq \varepsilon| u(x, t)\right|^{p}+C .
$$

Remark 1.5. When $\delta=0$, this result was already derived from the Liouville theorem in MZ98a and MZ00. It happens that adapting that proof to the case $\delta \neq 0$ is not straightforward, because the gradient structure is missing. However, unlike for the Liouville theorem, the adaptation is mainly technical. For the reader's convenience, we show in Section 4 how to adapt the proof of [MZ98a and MZ00 in the case $\delta \neq 0$.

Our paper is organized as follows: Section 2 and Section 3 are devoted to the proof of the Liouville theorem (we only prove Theorem 2 since Theorem 1 follows immediately from the self-similar transformation (1.9)). Note that Section 2 contains the main arguments with no details and Section 3 includes the whole proof with all the technical steps. Finally, we prove in Section 4 the applications to blow-up stated in Proposition 3

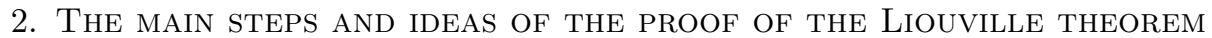

In this section, we adopt a pedagogical point of view and explain the main steps and ideas of the proof with no technical details. These details are presented in Section 3 The reader may think that our result is an interesting perturbation of the Liouville theorem proved in MZ00 for $\delta=0$. If this is true for the statement, it is certainly not the case for the proof for three structural reasons:

- The gradient structure breaks down when $\delta \neq 0$, which prevents us from using any energy method or blow-up criteria. To show blow-up, we need to find a very precise asymptotic behavior of the solution and show "by hand" that it cannot stay bounded.

- When $\delta \neq 0$, the linearized operator of (1.10) around the constant solution $w \equiv$ $\kappa$ is no longer selfadjoint and no general theory is applicable to derive eigenvalues directly. A careful decomposition of the solution is needed instead.

- Since equation (1.2) is invariant under rotations in the complex plane $(u \rightarrow$ $u e^{i \theta}$ ), this generates a null eigenvalue for the linear part of equation (1.10), and a precise modulation technique is needed, unlike the real-valued case when $\delta=0$.

The proof of the Liouville theorem is the same for $N=1$ and $N \geq 2$ with subcritical $p$ (see (1.3)). The only difference is in the multiplicity of the eigenvalues of the linearized operator of equation (1.10), which changes from 1 when $N=1$ to a higher value when $N \geq 2$. In particular, one needs some extra notation and careful linear algebra in higher dimensions. For the sake of clearness, we give here the proof when $N=1$. The interested reader may find in Section 4 (page 128) of MZ00 how to get the higher dimensional case from the case $N=1$. Clearly, the following statement is equivalent to Theorem 1. 
For any $M>0$, there exists $\delta_{0}^{\prime}(M)>0$ such that for all $|\delta| \leq \delta_{0}^{\prime}(M)$, if $w(y, s)$ is an entire solution of (1.10), defined for all $(y, s) \in \mathbb{R} \times \mathbb{R}$ and

$$
\|w(., s)\|_{L^{\infty}} \leq M
$$

then $w$ depends only on the variable $s$.

In the following, we will prove this latter statement. Let us consider $M>0$ and $w(y, s)$ satisfying (2.1) and prove that $w$ is trivial provided that $\delta$ is small. As in [MZ00, the starting point is the investigation of the behavior of $w(y, s)$ as $s \rightarrow-\infty$.

Part 1: Behavior of $w(y, s)$ as $s \rightarrow-\infty$.

In the case $\delta=0$, the method of Giga and Kohn GK85 proves that $w(y, s)$ approaches the set of stationary solutions of (1.10) $\left\{0, \kappa e^{i \theta} \mid \theta \in \mathbb{R}\right\}$ as $s \rightarrow-\infty$ in $L_{\rho}^{2}$. We would like to do the same here; that is why we give the stationary solutions of (1.10) in the following.

Proposition 2.1 ( $L^{\infty}$ stationary solutions of (1.10) $)$. Consider $\delta \neq 0$ and $v \in$ $L^{\infty}\left(\mathbb{R}^{N}\right)$ a solution of

$$
0=\Delta v-\frac{1}{2} y \cdot \nabla v-\frac{1+i \delta}{p-1} v+(1+i \delta)|v|^{p-1} v .
$$

Then, either $v \equiv 0$ or there exists $\theta_{0} \in \mathbb{R}$ such that $v \equiv \kappa e^{i \theta_{0}}$.

Remark 2.2. When $\delta=0$, the same result holds only for subcritical $p$ satisfying $(N-2) p \leq N+2$, and the proof due to Giga and Kohn is far from being trivial; see Theorem 1 (page 305) in GK85.

Proof of Proposition 2.1. Consider $v \in L^{\infty}\left(\mathbb{R}^{N}\right)$ a solution to (2.2). Multiplying (2.2) by $\bar{v} \rho$ and integrating over $\mathbb{R}^{N}$ gives after integration by parts

$$
0=-\int|\nabla v|^{2} \rho-\frac{(1+i \delta)}{p-1} \int|v|^{2} \rho+(1+i \delta) \int|v|^{p+1} \rho .
$$

Since $\delta \neq 0$, identifying the imaginary and the real parts gives $\int|\nabla v|^{2} \rho=0$; hence $\nabla v \equiv 0$ and $\Delta v \equiv 0$. Plunging this in (2.2) yields the result.

To prove that the solution approaches the set of stationary solutions, the method of Giga and Kohn breaks down, since it heavily relies on the existence of the following Lyapunov functional for equation (1.10) in the case $\delta=0$ :

$$
E(w)=\frac{1}{2} \int|\nabla w|^{2} \rho d y+\frac{1}{2(p-1)} \int|w|^{2} \rho d y-\frac{1}{p+1} \int|w|^{p+1} \rho d y .
$$

When $\delta \neq 0$, we don't have such a Lyapunov functional. Fortunately, a perturbation method used by Andreucci, Herrero and Velázquez works here and yields the following:

Proposition 2.3. For any $M>0$, there exists $\delta_{0}^{\prime}(M)$ such that if $|\delta| \leq \delta_{0}^{\prime}$ and $w$ is an arbitrary solution of (1.10) satisfying for all $(y, s) \in \mathbb{R} \times \mathbb{R},|w(y, s)| \leq M$, then either

$$
\text { (i) }\|w(., s)\|_{L_{\rho}^{2}} \rightarrow 0 \text { as } s \rightarrow-\infty \text { or }(\text { ii }) \inf _{\theta \in \mathbb{R}}\left\|w(., s)-\kappa e^{i \theta}\right\|_{L_{\rho}^{2}} \rightarrow 0 \text { as } s \rightarrow-\infty \text {. }
$$

The next parts of the strategy (parts 2 and 3) investigate cases (i) and (ii) of Proposition 2.3, which are certainly not of the same degree of difficulty. 
Part 2: Case where $w \rightarrow 0$ as $s \rightarrow-\infty$.

In this case, we have $w \equiv 0$. Rather than giving a proof, we simply explain here how the proof works. For the actual proof, we rely again on the method of Andreucci, Herrero and Velázquez (see Proposition 3.1, in section 3 of [AHV97]). Our argument is that the stationary solution of (1.10), which is identically zero, is stable in $L_{\rho}^{2}$; hence, no orbit can escape it, except the null orbit. To illustrate this, we write from equation (1.10) the following differential inequality for $h(s) \equiv \int_{\mathbb{R}}|w(y, s)|^{2} \rho(y) d y$,

$$
h^{\prime}(s) \leq-\frac{2}{p-1} h(s)+2 \int_{\mathbb{R}}|w(y, s)|^{p+1} \rho(y) d y .
$$

Using the regularizing effect of equation (1.10), we derive the following delay estimate:

$$
\forall s \in \mathbb{R}, \quad \int_{\mathbb{R}}|w(y, s)|^{p+1} \rho(d y) \leq C^{*}\left(\int_{\mathbb{R}}|w(y, s-1)|^{2} \rho(y) d y\right)^{\frac{p+1}{2}},
$$

for some positive $C^{*}$. Therefore,

$$
\forall s \in \mathbb{R}, h^{\prime}(s) \leq-\frac{2}{p-1} h(s)+C(M) h(s-1)^{\frac{p+1}{2}} .
$$

Using the fact that $h(s) \rightarrow 0$ as $s \rightarrow-\infty$ and delay ODE techniques, we show that $h(s)$ is driven by its linear part; hence for some $\varepsilon>0$ small enough, we have

$$
\forall \sigma \in \mathbb{R}, \forall s \geq \sigma+1, h(s) \leq \varepsilon_{0} e^{-\frac{2(s-\sigma)}{p-1}} .
$$

Fixing $s \in \mathbb{R}$ and letting $\sigma \rightarrow-\infty$, we get that $h(s) \equiv 0$; hence $w \equiv 0$.

Now that case (i) of Proposition 2.3 has been handled, we consider case (ii) in the following.

Part 3: Case where $\inf _{\theta \in \mathbb{R}}\left\|w(., s)-\kappa e^{i \theta}\right\|_{L_{\rho}^{2}} \rightarrow 0$ as $s \rightarrow-\infty$.

The question to be asked here is the following: Does the solution converge to a particular $\kappa e^{i \theta_{0}}$ as $s \rightarrow-\infty$ or not?

The key idea is to classify the $L_{\rho}^{2}$ behavior of $w$ as $s \rightarrow-\infty$. We proceed in 5 steps.

\section{Step 1: Formulation of the problem.}

Note that the degree of freedom in case (ii) of Proposition 2.3 comes from the invariance of equation (1.2) under the rotation $\left(u \rightarrow u e^{i \theta}\right)$. This invariance generates a zero mode for equation (1.10), which is difficult to control. The idea to gain this control and show the convergence of $w(y, s)$ is to use a modulation technique by introducing the following parametrization of the problem:

$$
w(y, s)=e^{i \theta(s)}(v(y, s)+\kappa) \text { with } \kappa=(p-1)^{-\frac{1}{(p-1)}} .
$$

A natural choice would be to take $\theta(s)$ such that

$$
\left\|w(., s)-e^{i \theta(s)} \kappa\right\|_{L_{\rho}^{2}}=\inf _{\theta \in \mathbb{R}}\left\|w(y, s)-\kappa e^{i \theta}\right\|_{L_{\rho}^{2}} .
$$

This is not our choice. We will instead choose $\theta(s)$ such that we kill the neutral mode mentioned above. More precisely, we claim the following:

Lemma 2.4. There exists $s_{1} \in \mathbb{R}$ and $\theta \in C^{1}\left(\left(-\infty, s_{1}\right], \mathbb{R}\right)$ such that

(i) $\forall s \leq s_{1}, \int(\operatorname{Im}(v)-\delta \operatorname{Re}(v)) \rho=0$, where $v$ is defined by (2.4).

(ii) We have $\|v(., s)\|_{L_{\rho}^{2}} \rightarrow 0$ as $s \rightarrow-\infty$. 
(iii) For all $s \leq s_{1}$, we have

$$
\left|\theta^{\prime}(s)\right| \leq C\|v(., s)\|_{L_{\rho}^{2}}^{2} .
$$

With the change of variables (2.4), we focus in the following steps on the description of the asymptotic behavior of $v(y, s)$ and $\theta(s)$ as $s \rightarrow-\infty$. Using (2.4), we write the equation satisfied by $v\left(=v_{1}+i v_{2}\right)$ as

$$
\begin{gathered}
\partial_{s} v=\tilde{\mathcal{L}} v-i \theta_{s}(v+\kappa)+G \\
\text { where } G=(1+i \delta)\left\{|v+\kappa|^{p-1}(v+\kappa)-\kappa^{p}-\frac{v}{p-1}-v_{1}\right\}
\end{gathered}
$$

satisfies $|G| \leq C|v|^{2}$ and $\left|G-(1+i \delta) \frac{1}{2 \kappa}\left\{(p-2) v_{1}^{2}+v_{2}^{2}+2 v_{1} v_{2}\right\}\right| \leq C|v|^{3}$.

A good understanding of our operator $\tilde{\mathcal{L}} v=\Delta v-\frac{1}{2} y \cdot \nabla v+(1+i \delta) v_{1}$ will be essential in our analysis. The following lemma provides us with the spectral properties of $\tilde{\mathcal{L}}$.

Lemma 2.5 (Eigenvalues of $\tilde{\mathcal{L}}$ ).

(i) $\tilde{\mathcal{L}}$ is an $\mathbb{R}$-linear operator defined on $L_{\rho}^{2}$ and its eigenvalues are given by

$$
\left\{1-\frac{m}{2} \mid m \in \mathbb{N}\right\} \text {. }
$$

Its eigenfunctions are given by $\left\{(1+i \delta) h_{m}, i h_{m} \mid m \in \mathbb{N}\right\}$, where

$$
h_{m}(y)=\sum_{n=0}^{\left[\frac{m}{2}\right]} \frac{m !}{n !(m-2 n) !}(-1)^{n} y^{m-2 n} .
$$

We have $\tilde{\mathcal{L}}\left((1+i \delta) h_{m}\right)=\left(1-\frac{m}{2}\right)(1+i \delta) h_{m}$ and $\tilde{\mathcal{L}}\left(i h_{m}\right)=-\frac{m}{2} i h_{m}$

(ii) Each $r \in L_{\rho}^{2}$ can be uniquely written as

$$
r(y)=(1+i \delta) \tilde{r}_{1}(y)+i \tilde{r}_{2}(y)=(1+i \delta)\left(\sum_{m=0}^{+\infty} \tilde{r}_{1 m} h_{m}(y)\right)+i\left(\sum_{m=0}^{+\infty} \tilde{r}_{2 m} h_{m}(y)\right),
$$

where:

$$
\begin{aligned}
& \tilde{r}_{1}(y)=\operatorname{Re}\{r(y)\} \text { and } \tilde{r}_{2}(y)=\operatorname{Im}\{r(y)\}-\delta \operatorname{Re}\{r(y)\} \\
& \text { and for } i=\{1,2\}, \tilde{r}_{i m}=\int \tilde{r}_{i}(y) \frac{h_{m}(y)}{\left\|h_{m}\right\|_{L_{\rho}^{2}}^{2}} \rho(y) d y .
\end{aligned}
$$

Remark 2.6. Note that the eigenvalues $1,1 / 2$ and 0 have a geometrical interpretation: they come from the invariance of equation (1.2) to translation in time $(\lambda=1)$ and space $(\lambda=1 / 2)$, dilations $u_{\lambda}(\xi, \tau) \rightarrow \lambda^{\frac{1}{p-1}} u(\xi \sqrt{\lambda}, \tau \lambda)$ and multiplications by $e^{i \theta}$ (the group $\mathcal{S}^{1}$ ) for $\lambda=0$.

Remark 2.7. Following (ii), we write each complex quantity (number or function) $z$ as $z=z_{1}+i z_{2}$ and $z=(1+i \delta) \tilde{z}_{1}+i \tilde{z}_{2}$ with $z_{j=1,2}, \tilde{z}_{j=1,2} \in \mathbb{R}$. In particular, we write

$$
\begin{aligned}
v(y, s) & =(1+i \delta) \tilde{v}_{1}(y, s)+i \tilde{v}_{2}(y, s) \\
& =(1+i \delta) \sum_{m=0}^{\infty} \tilde{v}_{1 m}(s) h_{m}(y)+i \sum_{m=0}^{\infty} \tilde{v}_{2 m}(s) h_{m}(y) .
\end{aligned}
$$


Proof. Using the notation (2.11), we see that

$$
\tilde{\mathcal{L}} v=\left(\begin{array}{ll}
\mathcal{L} & 0 \\
0 & \mathcal{L}-\mathcal{I}
\end{array}\right)\left(\begin{array}{l}
\tilde{v}_{1} \\
\tilde{v}_{2}
\end{array}\right)
$$

where

$$
\mathcal{L} h=\Delta h-\frac{1}{2} y \cdot \nabla h+h
$$

is a well-known selfadjoint operator of $L_{\rho}^{2}(\mathbb{R}, \mathbb{R})$ whose eigenfunctions are $h_{m}(2.9)$, which are dilations of Hermite polynomials. Thus, the spectral properties of $\tilde{\mathcal{L}}$ directly derive from those of $\mathcal{L}$. The interested reader may find details in Lemma 2.2, page 590, from Zaag [Zaa98].

Note from this lemma that operator $\tilde{\mathcal{L}}$ has three nonnegative eigenvalues:

- $\lambda=1$, with eigenfunction $(1+i \delta) h_{0}(y)=(1+i \delta)$.

- $\lambda=1 / 2$, with eigenfunction $(1+i \delta) h_{1}(y)=(1+i \delta) y$.

- $\lambda=0$, with two eigenfunctions $(1+i \delta) h_{2}(y)=(1+i \delta)\left(y^{2}-2\right)$ and $i h_{0}(y)=i$.

From (2.11) and (2.10), the coordinate of $v(y, s)$ along the direction $i h_{0}$ is

$$
\begin{array}{r}
\tilde{v}_{20}(s)=\int(\operatorname{Im}(v(y, s))-\delta \operatorname{Re}(v(y, s))) \frac{h_{0}(y)}{\left\|h_{0}\right\|_{L_{\rho}^{2}}^{2}} \rho(y) \\
=\int(\operatorname{Im}(v(y, s))-\delta \operatorname{Re}(v(y, s))) \rho(y) .
\end{array}
$$

Using (i) of Lemma 2.4, we see that the choice of $\theta(s)$ guarantees that

$$
\forall s \leq s_{1}, \tilde{v}_{20}(s)=0 .
$$

In the next step, we will use the spectral information of $\tilde{\mathcal{L}}$ to derive the asymptotic behavior of $v$, then $w$ as $s \rightarrow-\infty$.

Step 2: Asymptotic behavior as $s \rightarrow-\infty$.

As $s \rightarrow-\infty$, we expect that the coordinates of $v$ on the eigenfunctions for $\lambda \geq 0$ will dominate. These eigenfunctions are $(1+i \delta)$ when $\lambda=1,(1+i \delta) y$ when $\lambda=1 / 2$, $(1+i \delta)\left(y^{2}-2\right)$ or $i$ when $\lambda=0$. Note that for this latter case, the direction along $i$ already vanishes thanks to the choice of $\theta(s)$ (see (2.14) $)$. So, if $\lambda=0$ dominates, that is, the coordinate of $v$ on $(1+i \delta)\left(y^{2}-2\right)$ dominates, since the linear part vanishes, the equation is driven by the quadratic approximation $\dot{x} \sim-x^{2}$, that is, $x \sim \frac{1}{s}$. Using (iii) of Lemma 2.4 we see that $\theta(s)$ has a limit as $s \rightarrow-\infty$; hence $w$ converges from (2.4). More precisely, we have:

Proposition 2.8. There exists $\theta_{0} \in \mathbb{R}$ such that $\theta(s) \rightarrow \theta_{0}$ and $\left\|w(., s)-\kappa e^{i \theta_{0}}\right\|_{L_{\rho}^{2}}$ $\rightarrow 0$ as $s \rightarrow-\infty$. More precisely, one of the following situations occurs as $s \rightarrow-\infty$, for some $C_{0} \in \mathbb{R}$ and $C_{1} \in \mathbb{R}^{*}$,

$$
\begin{array}{ll}
\text { (i) }\left\|w(., s)-\left\{\kappa+(1+i \delta) C_{0} e^{s}\right\} e^{i \theta_{0}}\right\|_{L_{\rho}^{2}} & \leq C e^{\frac{3}{2} s}, \\
\text { (ii) }\left\|w(., s)-e^{i \theta_{0}}\left\{\kappa-(1+i \delta) \frac{\kappa}{4\left(p-\delta^{2}\right) s}\left(y^{2}-2\right)-i \frac{\left(1+\delta^{2}\right) \delta \kappa^{2}}{2\left(p-\delta^{2}\right)^{2}} \frac{1}{s}\right\}\right\|_{L_{\rho}^{2}} & \leq C \frac{\log |s|}{s^{2}} \\
\text { (iii) }\left\|w(., s)-\left\{\kappa+(1+i \delta) C_{1} e^{s / 2} y\right\} e^{i \theta_{0}}\right\|_{L_{\rho}^{2}} & \leq C e^{(1-\varepsilon) s} .
\end{array}
$$

In Step 3, we show that case (i) yields the explicit solution $\varphi_{\delta}\left(s-s_{0}\right)$ for some $s_{0}$. In Steps 4 and 5 , we rule out cases (ii) and (iii).

In comparison with the case $\delta=0$, we can say that the difficulty in deriving Proposition 2.8 is only technical. One should bear in mind that the difficulty level 
is much lower than the obstacles we have in Steps 4 and 5 to rule out cases (ii) and (iii) of Proposition 2.8

\section{Step 3: Case where (i) holds.}

As for Step 2, there is no real novelty in this step; the difficulty is purely technical. First we recall (i) from Proposition 2.8 .

$$
\left\|w(., s)-\left\{\kappa+(1+i \delta) C_{0} e^{s}\right\} e^{i \theta_{0}}\right\|_{L_{\rho}^{2}} \leq C e^{\frac{3}{2} s} .
$$

Let us remark that we already have a solution $\hat{\varphi}(s) e^{i \theta_{0}}$ of (1.10) defined in $\mathbb{R} \times$ $(-\infty, \hat{s}]$ for some $\hat{s} \in \mathbb{R}$ and which satisfies the same expansion as $w$ :

$$
\begin{aligned}
& \text { (a) if } C_{0}=0, \text { just take } \hat{\varphi} \equiv \kappa, \\
& \text { (b) if } C_{0}<0, \text { take } \hat{\varphi} \equiv \varphi_{\delta}\left(s-s_{0}\right) \text {, where } s_{0}=-\log \left(-\frac{C_{0}(p-1)}{\kappa}\right), \\
& \text { (c) if } C_{0}>0, \text { take } \hat{\varphi} \equiv \varphi_{\delta}^{*}\left(s-s_{0}\right) \text {, where } s_{0}=\log \left(\frac{C_{0}(p-1)}{\kappa}\right) \\
& \text { and } \varphi_{\delta}^{*}(s)=\kappa\left(1-e^{s}\right)^{-\frac{(1+i \delta)}{(p-1)}} .
\end{aligned}
$$

$\varphi_{\delta}^{*}(s)$ is a solution of (1.10) that blows up at $s=0$, but is bounded for all $s \leq-1$. Note that, from (2.16) we have

$$
\left\|w(., s)-\hat{\varphi}(s) e^{i \theta_{0}}\right\|_{L_{\rho}^{2}} \leq C e^{\frac{3}{2} s} .
$$

Since the difference between the two solutions of (1.10) is of order $e^{3 s / 2}$ and the largest eigenvalue of $\tilde{\mathcal{L}}$ is $1<\frac{3}{2}$, this difference has to vanish leading to $w(y, s)=$ $\hat{\varphi}(s) e^{i \theta_{0}}$ (remember that the largest eigenvalue matters, since $s \rightarrow-\infty$ ). Since case (c) violates the uniform bound (2.1), only cases (a) or (b) occur. More precisely, we have the following:

Proposition 2.9. Assume that case (i) of Proposition 2.8 holds. Then, either $w \equiv$ $\kappa e^{i \theta_{0}}$ or there exists $s_{0} \in \mathbb{R}$ such that for all $(y, s) \in \mathbb{R} \times \mathbb{R}, w(y, s)=\varphi_{\delta}\left(s-s_{0}\right) e^{i \theta_{0}}$ for some $\theta_{0} \in \mathbb{R}$.

Steps 4 and 5: Irrelevance of cases (ii) and (iii) of Proposition 2.8 .

Step 4 and the following account for the novelty of our work. Indeed, in the case $\delta=0$ treated in [MZ00], cases (ii) and (iii) of Proposition 2.8 were ruled out thanks to a blow-up criterion based on energy methods. Indeed, when $\delta=0$, Merle and Zaag used the Lyapunov functional for equation (1.10) introduced in (2.3). More precisely, they have the following blow-up criterion (see Proposition 2.1, page 111 in [MZ00]):

Lemma 2.10 (A blow-up criterion for equation (1.10) when $\delta=0$ ). Let $W$ be a solution of (1.10) (with $\delta=0$ ), which satisfies:

$$
E\left(W\left(y, s_{0}\right)\right)<\frac{p-1}{2(p+1)}\left(\int_{\mathbb{R}^{N}}\left|W\left(y, s_{0}\right)\right|^{2} \rho(y) d y\right)^{\frac{p+1}{2}},
$$

for some $s_{0} \in \mathbb{R}$. Then, $W$ blows up at some time $S>s_{0}$.

Still for $\delta=0$, it is shown in MZ00, when case (ii) or (iii) hold in Proposition 2.8, that

for some $a_{0}$ and $s_{0}$, we have

$$
E\left(w_{a_{0}}\left(., s_{0}\right)\right)<\frac{p-1}{2(p+1)}\left(\int w_{a_{0}}\left(y, s_{0}\right)^{2} \rho(y) d y\right)^{\frac{p+1}{2}},
$$


where

$$
w_{a_{0}}(y, s)=w\left(y+a_{0} e^{\frac{s}{2}}, s\right)
$$

is also a solution of (1.10).

A contradiction then follows since, in the same time, $w_{a_{0}}$ is defined for all $s \in \mathbb{R}$ from (2.20) and has to blow up by condition (2.19) and Lemma 2.10.

When $\delta \neq 0$, all this collapses. No perturbation method can allow us to use in any sense the Lyapunov functional or the blow-up criterion. We have to invent a new method to rule out cases (ii) and (iii) of Proposition 2.8. Let us explain our strategy only for case (ii), since it is quite similar for case (iii). From the rotation invariance of equation (1.10), we assume that $\theta_{0}=0$.

Our source of inspiration is the study of (1.10), when $\delta=0$ and $w \rightarrow \kappa$ as $s \rightarrow+\infty$ (and not $-\infty$ ) by Herrero and Velázquez [HV93] and Velázquez Vel92, to obtain the (supposed to be generic) profile, starting with the following profile:

$$
w(y, s)=\kappa+\frac{\kappa}{2 p s}\left(1-\frac{1}{2}|y|^{2}\right)+o\left(\frac{1}{s}\right) \text { as } s \rightarrow \infty .
$$

The convergence here takes place in $L_{\rho}^{2}$ and $L^{\infty}(|y|<R)$ for any $R>0$.

Herrero and Velázquez extended this convergence to a larger set of the form $|y| \leq K \sqrt{s}$, for any $K>0$. They obtained:

$$
\sup _{|y|<K \sqrt{s}}\left|w(y, s)-f_{0}\left(\frac{y}{\sqrt{s}}\right)\right| \rightarrow 0 \text { as } s \rightarrow+\infty
$$

where $f_{0}=\left((p-1)+\frac{(p-1)^{2}}{4 p} \frac{|y|^{2}}{s}\right)^{-\frac{1}{p-1}}$ is a solution of

$$
0=\frac{1}{2} \xi \cdot \nabla f_{0}(\xi)-\frac{1}{p-1} f_{0}(\xi)+\left|f_{0}\right|^{p-1} f_{0}(\xi), \text { where } \xi=\frac{y}{\sqrt{s}} .
$$

In some sense, we can say that $f_{0}$ is an approximate solution of (1.10) when $s \rightarrow \infty$, because

$$
\left\|\partial_{s} f_{0}-\left\{\Delta f_{0}+\frac{1}{2} \xi \cdot \nabla f_{0}-\frac{1}{p-1} f_{0}+\left|f_{0}\right|^{p-1} f_{0}\right\}\right\|_{L^{\infty}}=\left\|\partial_{s} f_{0}-\Delta f_{0}\right\|_{L^{\infty}} \leq \frac{C}{s} .
$$

We note that Velázquez's method is a kind of method of characteristic applied to the parabolic equation (1.10), where the Laplacian term is dropped down because the profile is flat. Here, we will use ideas from Velázquez to find the profiles of the solution in the variables $\frac{y}{\sqrt{-s}}\left(y e^{s / 2}\right.$ in Step 5). We hope to find singular profiles, which violate the upper bound (2.1) on $w(y, s)$. Our candidate for the profile is $G\left(\frac{y}{\sqrt{-s}}\right)$, with $G(\xi)=\kappa\left(1-\frac{(p-1)}{4\left(p-\delta^{2}\right)} \xi^{2}\right)^{-\frac{(1+i \delta)}{(p-1)}}$. In fact $G$ is a solution of

$$
0=-\frac{1}{2} \cdot \xi \nabla G(\xi)-\frac{1+i \delta}{p-1} G+(1+i \delta)|G|^{p-1} G .
$$

We can note (as in the case of $f_{0}$ defined below) that $G$ is an approximate solution of (1.10) (for $|y|<K_{0} \sqrt{-s}$, where $K_{0}=\sqrt{\frac{4\left(p-\delta^{2}\right)}{(p-1)}}$ ). We see also that $G$ is singular for $|y|=K_{0} \sqrt{-s}$. Using Velázquez's technique to extend the convergence in (ii) of Proposition 2.8 from $|y|<R$ to larger regions $|y|<\varepsilon_{0} \sqrt{-s}$, with $\varepsilon_{0}<K_{0}$, we can prove the following. 
Proposition 2.11. Assume that case (iii) from Proposition 2.8 holds. Then there exists $a_{0}>0$, such that:

$$
\begin{array}{r}
\lim _{s \rightarrow-\infty} \sup _{|y| \leq \varepsilon_{0} \sqrt{-s}}\left|w(y, s)-G\left(\frac{y}{\sqrt{-s}}\right)\right|=0, \\
\text { where } G(\xi)=\kappa\left(1-\frac{(p-1)}{4\left(p-\delta^{2}\right)} \xi^{2}\right)^{-\frac{(1+i \delta)}{(p-1)}} .
\end{array}
$$

Imagine for a second that (2.21) holds for any arbitrary $\varepsilon_{0}<K_{0}$. Since $|G(\xi)| \rightarrow$ $\infty$ as $\xi \rightarrow K_{0}$, we can fix $\varepsilon_{0}$ large enough so that $\left|G\left(\varepsilon_{0}\right)\right| \geq 3 M$. Taking $\left|s_{0}\right|$ large enough in (2.21), we then see that

$$
\left|w\left(\varepsilon_{0} \sqrt{-s_{0}}, s_{0}\right)\right| \geq 2 M,
$$

which contradicts the upper bound (2.1). It happens that, unlike the case $s \rightarrow \infty$, where $\xi=0$ realizes the maximum of the profile $f_{0}$, here $\xi=0$ realizes the minimum, which obliges us to take $\varepsilon_{0}$ small enough in order to use Velázquez' method of convergence extension. Since $\varepsilon_{0}$ is small in our approach, we remark from (2.21) that $w(y, s)$ is flat (i.e. close to a constant) in a large region, in the sense that

$$
\sup _{\left|y-\frac{\varepsilon_{0}}{2} \sqrt{-s_{0}}\right| \leq 4\left|s_{0}\right|^{1 / 4}}\left|w\left(y, s_{0}\right)-G\left(\frac{\varepsilon_{0}}{2}\right)\right| \rightarrow 0 \text { as } s_{0} \rightarrow-\infty .
$$

Using a kind of continuity with respect to initial data for equation (1.10), we can show that for any $\varepsilon>0$,

$$
\sup _{s_{0} \leq s \leq s_{0}^{*}-\varepsilon}\left|w\left(\frac{\varepsilon_{0}}{2} \sqrt{-s_{0}}, s\right)-W_{\varepsilon_{0}}(s)\right| \rightarrow 0 \text { as } s_{0} \rightarrow \infty,
$$

where $s_{0}^{*}<+\infty$ is the lifespan of $W_{\varepsilon_{0}}(s)$, the space independent solution of (1.10), with $W_{\varepsilon_{0}}\left(s_{0}\right) \equiv G\left(\frac{a_{0}}{2}\right)$. It happens that $W_{a_{0}}$ can be computed explicitly:

$$
W_{\varepsilon_{0}}(s)=\kappa\left(1-e^{s-s_{0}} \frac{(p-1) \varepsilon_{0}^{2}}{16\left(p-\delta^{2}\right)}\right)^{-\frac{(1+i \delta)}{p-1}}
$$

and that it blows up at time $s=s_{0}^{*}-\log \left(\frac{(p-1) \varepsilon_{0}^{2}}{16\left(p-\delta^{2}\right)}\right)>s_{0}$, because $\varepsilon_{0}$ is small.

Taking $s_{0}^{*}=s_{0}-\mu_{0}$, where $\mu_{0}>0$ is small enough such that $\left|W_{a_{0}}\left(s_{0}^{*}-\mu_{0}\right)\right| \geq 3 M$, we see from (2.22) that $\left|w\left(\frac{\varepsilon_{0}}{2} \sqrt{-s_{0}}, s_{0}^{*}-\mu_{0}\right)\right| \geq 2 M$, which violates the upper bound (2.1).

\section{Conclusion of Part 3 and sketch of proof of the Liouville theorem:}

From Steps 4 and 5 we see that cases (ii) and (iii) of Proposition 2.8 are ruled out. By Step 3 , we obtain that $w \equiv \kappa e^{i \theta_{0}}$ or $w \equiv \varphi_{\delta}\left(s-s_{0}\right) e^{i \theta_{0}}$ for some real $s_{0}$ and $\theta_{0}$, where $\varphi_{\delta}$ is defined in Theorem 1, which is the desired conclusion of Theorem 1. In Section 3, we give the details of the proof.

\section{Details of the proof of the Liouville theorem}

In this section, we give the whole proof of the Liouville theorem. We only prove Theorem 1 since Theorem 2 immediately follows through the self-similar transformation (1.9). Note that in Section 2, we already gave a sketch of the proof stressing only the main arguments. Thus this section is intended only for readers interested in technical details.

We adopt here the same sectioning as in Section 2: three parts and Part 3 is divided into five steps. Hence, we recommend that the reader first reads a given step in Section 2 before reading the corresponding step in Section 3. As in Section 
2, we prove Theorem 1 in its form given in the statement around (2.1). We consider $M>0$ and a global solution $w(y, s)$ of (1.10), defined for all $(y, s) \in \mathbb{R} \times \mathbb{R}$ such that

$$
\|w(y, s)\|_{L^{\infty}} \leq M
$$

Our goal is to find $\delta_{0}^{\prime}(M)>0$, such that if $|\delta| \leq \delta_{0}^{\prime}(M)$, w depends only on the variable $s$. We proceed in three parts:

In Part 1 , we show that when $s \rightarrow-\infty$, either $w \rightarrow 0$ or $w$ approaches the set $\left\{\kappa e^{i \theta} \mid \theta \in \mathbb{R}\right\}$.

In Part 2, we handle the first case and show that $w \equiv 0$.

In Part 3, we linearize the equation around $\kappa e^{i \theta(s)}$, for some well-chosen $\theta(s)$, and show that either $w \equiv \kappa e^{i \theta_{0}}$ or $w \equiv \varphi_{\delta}\left(s-s_{0}\right) e^{i \theta_{0}}$ for some real $s_{0}$ and $\theta_{0}$, where $\varphi_{\delta}$ is defined in Theorem 1, which concludes the proof.

It happens that we rely on the analysis performed by Andreucci, Herrero and Velázquez [AHV97 for the system (1.7). That is the reason why we give Part 1 and Part 2 at once.

Parts 1 and 2: Behavior of $w(y, s)$ as $s \rightarrow \infty$ and conclusion in the case where $w \rightarrow 0$ as $s \rightarrow-\infty$.

In these parts, we investigate the behavior of $w$ as $s \rightarrow-\infty$ and reach a conclusion in the easiest case. Following what we wrote in Part 1 of Section 2, we know from Proposition 2.1 that the set of stationary solutions of (1.10) consists of 0 and $\kappa e^{i \theta}$, where $\theta \in \mathbb{R}$. In order to prove that $w$ approaches this set as $s \rightarrow-\infty$, we rely completely on the analysis performed in AHV97 for the system (1.7). Indeed, no extra arguments are necessary for the present equation (1.10). That is why we only give the main arguments which make the proof of AHV97 hold for equation (1.10) and refer the interested reader to AHV97] for the details. Now, using the perturbation method of [AHV97, we have the following:

Proposition 3.1 (A primary classification). For any $M>0$, there exists $\delta_{0}^{\prime}(M)$ such that if $|\delta| \leq \delta_{0}^{\prime}$ and $w$ is an arbitrary solution to (1.10) satisfying for all $(y, s) \in$ $\mathbb{R} \times \mathbb{R},|w(y, s)| \leq M$, then, either $(i)(\|w(s)\| \equiv 0)$ or $(i i)\left(\inf _{\theta \in \mathbb{R}}\left\|w(s)-\kappa e^{i \theta}\right\| \rightarrow 0\right)$ as $s \rightarrow-\infty$.

Remark 3.2. This result replaces Proposition 2.3 and Part 2 in Section 2.

Remark 3.3. In AHV97, the conclusion of the authors in Theorem 2 for system (1.7) is more accurate: either $(\Phi, \Psi)$ is $(0,0)$ or $(\Gamma, \gamma)$ defined in (1.8), or

$$
(\Phi, \Psi) \rightarrow(\Gamma, \gamma) \text { at }-\infty \text { and }(\Phi, \Psi) \rightarrow(0,0) \text { at }+\infty .
$$

Using the same technique for our equation (1.10), we get Proposition 3.1 Indeed, due to the fact that the set of nontrivial stationary solutions is a continuum (see Proposition 2.1), we need a modulation technique to derive the case $w \equiv \kappa e^{i \theta}$. This case will be treated in Part 3.

Proof of Proposition 3.1. This proposition follows from the arguments developed for the twin system (1.7) in [AHV97, and no more. To limit our paper to a reasonable length, we don't give the proof. However, we should mention the 3 fundamental features of (1.10) that one needs to check to be convinced that the proof of Andreucci, Herrero and Velázquez works here.

- Both systems are of parabolic type involving the same linear operator

$$
\mathcal{L}_{0} v=\frac{1}{\rho} \operatorname{div}(\rho \nabla v)=\Delta v-\frac{1}{2} y \cdot \nabla v .
$$


If $v=(1+i \delta) \tilde{v}_{1}+i \tilde{v}_{2}$ with $\tilde{v}_{1}, \tilde{v}_{2} \in \mathbb{R}$, then

$$
\begin{aligned}
& \partial_{s} \tilde{v}_{1}=\mathcal{L}_{0} \tilde{v}_{1}-a \tilde{v}_{1}+f_{1}\left(\tilde{v}_{1}, \tilde{v}_{2}\right), \\
& \partial_{s} \tilde{v}_{2}=\mathcal{L}_{0} \tilde{v}_{2}-b \tilde{v}_{2}+f_{2}\left(\tilde{v}_{1}, \tilde{v}_{2}\right)
\end{aligned}
$$

with $a<0, b<0$ and $\left|f_{i}\left(\tilde{v}_{1}, \tilde{v}_{2}\right)\right| \leq C|v|^{\alpha}$ for some $\alpha>1$.

- When $p=q=p_{0}$ in (1.7), the authors give in (3.12) and Lemma 3.2 of AHV97 a classification of entire solutions. In our case, when $\delta=0$ in (1.10), we have the following Liouville theorem (see Theorem 1 in [MZ00]).

Proposition 3.4 (Merle-Zaag [MZ00]; A Liouvile theorem for equation (1.10) with $\delta=0$ and subcritical $p)$. Assume (1.3) and let $w \in L^{\infty}\left(\mathbb{R}^{N} \times \mathbb{R}, \mathbb{C}\right)$ be a solution of

$$
\frac{\partial w}{\partial s}=\Delta w-\frac{1}{2} y \cdot \nabla w-\frac{w}{p-1}+|w|^{p-1} w .
$$

Then, necessarily, one of the following cases occurs:

a) $w \equiv 0$,

b) $\exists \theta \in \mathbb{R}$ such that $w(y, s)=\kappa e^{i \theta}$,

c) there exists $s_{0} \in \mathbb{R}$, such that for all $(y, s) \in \mathbb{R}^{N} \times \mathbb{R}, w(y, s)=\varphi\left(s-s_{0}\right) e^{i \theta_{0}}$, where $\theta_{0} \in \mathbb{R}$ and

$$
\varphi(s)=\kappa\left(1+e^{s}\right)^{-\frac{1}{p-1}} .
$$

Remark 3.5. Note that $\varphi$ is the unique global solution (up to a translation) of

$$
\varphi_{s}=-\frac{\varphi}{p-1}+\varphi^{p}
$$

satisfying $\varphi \rightarrow \kappa$ as $s \rightarrow-\infty$ and $\varphi \rightarrow 0$ as $s \rightarrow \infty$. The method of Andreucci, Herrero and Velázquez in AHV97 is in fact a perturbation method around this result.

The property of equation (1.2) saying "small $L_{\rho}^{2}$ norm implies no blow-up locally" (note that this property replaces the Giga-Kohn property "small local energy implies no blow-up locally", which breaks down because we no longer have a gradient structure) becomes the property:

Proposition 3.6. For all $M>0$, there exist positive $\eta_{0}, C_{0}$ and $M_{0}$ such that if $|\delta| \leq 1$ and $v$ is a solution of (1.2) satisfying

$$
\forall t \in[0,1), \quad\|v(t)\|_{L^{\infty}} \leq M(1-t)^{-\frac{1}{p-1}} \text { and if } \forall\left|x_{0}\right| \leq 1,\left\|w_{x_{0}}(., 0)\right\|_{L_{\rho}^{2}} \leq \eta
$$

for some $0<\eta \leq \eta_{0}$, where

$$
y=\frac{x-x_{0}}{\sqrt{1-\tau}}, s=-\log (1-\tau), w_{x_{0}}(y, s)=(1-\tau)^{\frac{1+i \delta}{p-1}} v(\xi, \tau),
$$

then:

(i) for all $\left|x_{0}\right| \leq 1$ and $s \in[0,+\infty)$,

$$
\left\|w_{x_{0}}(., s)\right\|_{L_{\rho}^{2}} \leq C_{0} \eta e^{-\frac{s}{p-1}},
$$

(ii) for all $|x| \leq 1$ and $t \in[0,1)$, we have $|v(x, t)| \leq M_{0}$.

Now, we write the following lemma, which will be useful in the proof of the proposition above. 
Lemma 3.7 (Regularizing effect of the operator $\mathcal{L}$ ). Assume that $\psi(y, s)$ satisfies $\forall s \in[a, b], \quad \forall y \in \mathbb{R}, \quad \psi_{s} \leq(\mathcal{L}+\sigma) \psi$ and $0 \leq \psi(y, s)$,

for some $a \leq b$ and $\sigma \in \mathbb{R}$, where

$$
\mathcal{L} \psi=\Delta \psi-\frac{1}{2} y \cdot \nabla \psi+\psi=\frac{1}{\rho} \operatorname{div}(\rho \nabla \psi)+\psi .
$$

Then for any $r>1$, there exists $C^{*}=C^{*}(r, \sigma)>0$ and $s^{*}=s^{*}(r)>0$ such that

$$
\forall s \in\left[a+s^{*}, b\right],\left(\int_{\mathbb{R}}|\psi(y, s)|^{r} \rho(y) d y\right)^{1 / r} \leq C^{*}\left\|\psi\left(., s-s^{*}\right)\right\|_{L_{\rho}^{2}} .
$$

Proof. See Lemma 2.3 in Vel93.

Proof of Proposition 3.6. Consider $M>0,|\delta| \leq 1$ and a solution $v$ of (1.2) such that (3.1) holds for some $\eta>0,\left|x_{0}\right| \leq 1$.

(i) For simplicity, we write $w$ instead of $w_{x_{0}}$. Since $w$ is a solution of (1.10), we multiply (1.10) by $w \rho$ and integrate to get

$$
I^{\prime}(s) \leq-\frac{2}{p-1} I(s)+\int|w(y, s)|^{p+1} \rho(y) d y, \text { where } I(s)=\int|w(y, s)|^{2} \rho(y) d y .
$$

If we note that $w=w_{1}+i w_{2}$, then using Kato's inequality $\left(\Delta w_{i} \cdot \operatorname{sgn}\left(w_{i}\right) \leq \Delta\left|w_{i}\right|\right.$ with $i=1,2)$ and the fact that $w$ is bounded, we obtain by equation (1.10)

$$
\partial_{s}\left(\left|w_{1}\right|+\left|w_{2}\right|\right) \leq \Delta\left(\left|w_{1}\right|+\left|w_{2}\right|\right)-\frac{y}{2} \cdot \nabla\left(\left|w_{1}\right|+\left|w_{2}\right|\right)+C\left(\left|w_{1}\right|+\left|w_{2}\right|\right),
$$

for some $C=C(M)>0$.

Using Lemma 3.7, we see that there exist $C^{*}(M)>0$ and $s^{*}=s^{*}(p+1)>0$ such that for all $s \geq s^{*}$,

$$
\int|w(y, s)|^{p+1} \rho(y) d y \leq C^{*}\left\|w\left(., s-s^{*}\right)\right\|_{L_{\rho}^{2}}^{p+1} .
$$

Now, we divide the proof into two steps:

Step 1: $0 \leq s \leq s^{*}$. Using (3.5) and the fact that $w$ is bounded by $M>0$ (see (3.1)), we get

$$
I^{\prime}(s) \leq \lambda I(s) \text { for some } \lambda=\lambda(M)>0 ;
$$

hence $I(s) \leq e^{\lambda s} I(0) \leq e^{\lambda s} \eta^{2} \leq \frac{C_{0}^{2}}{2} \eta^{2} e^{-\frac{2 s}{p-1}}$, where we define $C_{0}^{2}=2 e^{\left(\lambda+\frac{2}{p-1}\right) s^{*}}$. This gives (3.2) for $0 \leq s \leq s^{*}$.

Step 2: $s \geq s^{*}$. In this step, we argue by contradiction to prove (3.2) for all $s \geq s^{*}$. We suppose that there exists $s_{1}>s^{*}$ such that

$$
\begin{aligned}
I(s) & <\left(C_{0} \eta\right)^{2} e^{-\frac{2 s}{p-1}}, \text { for all } s^{*} \leq s<s_{1}, \\
I\left(s_{1}\right) & =\left(C_{0} \eta\right)^{2} e^{-\frac{2 s_{1}}{p-1}} .
\end{aligned}
$$

Let $F(s)=I(s)\left(C_{0} \eta\right)^{-2} e^{\frac{2 s}{p-1}}$. From (3.5), (3.6), (3.7) and Step 1, we have for all $s^{*} \leq s \leq s_{1}$,

$$
\begin{aligned}
F^{\prime}(s) & \leq C^{*}\left(C_{0} \eta\right)^{-2} e^{\frac{2 s}{p-1}} I\left(s-s^{*}\right)^{\frac{p+1}{2}} \\
& \leq C^{*}\left(C_{0} \eta\right)^{p-1} e^{\frac{2 s}{p-1}} e^{-\left(s-s^{*}\right) \frac{p+1}{p-1}} \leq C^{*}\left(C_{0} \eta\right)^{p-1} e^{\frac{p+1}{p-1} s^{*}} e^{-s} .
\end{aligned}
$$


Since $F\left(s^{*}\right) \leq \frac{1}{2}$ from the step above, we integrate the last inequality to obtain

$$
\begin{aligned}
F\left(s_{1}\right) & \leq C^{*}(C \eta)^{p-1} e^{\frac{p+1}{p-1} s^{*}}\left(e^{-s^{*}}-e^{-s_{1}}\right)+F\left(s^{*}\right) \\
& \leq C^{*}(C \eta)^{p-1} e^{\frac{2 s^{*}}{p-1}}+\frac{1}{2} \leq \frac{3}{4}
\end{aligned}
$$

for $\eta \leq \eta_{0}(M)$ small enough. This contradicts (3.8). Therefore, (3.2) holds.

(ii) Applying parabolic regularity to equation (1.10) and using estimate (3.1), we get for all $\left|x_{0}\right| \leq 1, R>0$ and $|y|<R,\left|w_{x_{0}}(y, s)\right| \leq M_{0} e^{-\frac{s}{p-1}}$; hence for all $t \in[0,1),\left|v\left(x_{0}, t\right)\right| \leq M_{0}$, for some $M_{0}=M_{0}(M)$. This ends the proof of Proposition 3.6 .

Part 3: Case where $\inf _{\theta \in \mathbb{R}}\left\|w(., s)-\kappa e^{i \theta}\right\|_{L_{\rho}^{2}} \rightarrow 0$ as $s \rightarrow-\infty$. We study case (ii) of Proposition 3.1. As we wrote in Part 3 of Section 2, the natural question is to know whether $w$ converges to a particular $\kappa e^{i \theta_{0}}$ as $s \rightarrow-\infty$ or not. A modulation technique will be essential to classify the $L_{\rho}^{2}$ behavior for $w$ and prove the convergence. We proceed in five steps.

- Step 1 is intended to be the modulation technique.

- In Step 2, we show that the linearized problem of (1.10) around $\kappa e^{i \theta}$ has 3 nonnegative directions as $s \rightarrow-\infty(\lambda=1,1 / 2$ or 0$)$, and that the component along one direction dominates the others. This gives a kind of profile for $w$ with a uniform convergence on every compact set.

- In Step 3, we show that the case where $\lambda=1$ dominates corresponds either to $w=\kappa e^{i \theta_{0}}$ or $w=\varphi_{\delta}\left(s-s_{0}\right) e^{i \theta_{0}}$ for some $\theta_{0} \in \mathbb{R}$ and $s_{0} \in \mathbb{R}$, where $\varphi_{\delta}$ is defined in Theorem 1.

- Steps 4 and 5: To rule out cases where the directions $\lambda=1 / 2$ or $\lambda=0$ dominate, we use a geometrical method where the key idea is Velázquez's work Vel92 to extend the convergence from compact sets to larger zones, where the profile appears to be singular, which violates the uniform bound (2.1) in $w$. These steps are innovative to our work.

Step 1: Formulation of the problem.

Let us recall Lemma 2.4 from Section 2.

Lemma 2.4. There exists $s_{1} \in \mathbb{R}$ and $\theta \in C^{1}\left(\left(-\infty, s_{1}\right], \mathbb{R}\right)$ such that

(i) $\forall s \leq s_{1}, \int(\operatorname{Im}(v)-\delta \operatorname{Re}(v)) \rho=0$, where $v$ is defined by

$$
w(y, s)=e^{i \theta(s)}(v(y, s)+\kappa) \text { with } \kappa=(p-1)^{-\frac{1}{(p-1)}} .
$$

(ii) We have $\|v(., s)\|_{L_{\rho}^{2}} \rightarrow 0$ as $s \rightarrow-\infty$.

(iii) For all $s \leq s_{1}$, we have

$$
\left|\theta^{\prime}(s)\right| \leq C\|v(., s)\|_{L_{\rho}^{2}}^{2} .
$$

Proof of Lemma 2.4. (i) Since $\inf _{\theta \in[0,2 \pi]}\left\|w(., s)-\kappa e^{i \theta}\right\|_{L_{\rho}^{2}} \rightarrow 0$ as $s \rightarrow-\infty$ and $\left\|w(., s)-\kappa e^{i \theta}\right\|_{L_{\rho}^{2}}$ is continuous as a function of $\theta$ and $w$, there exists $\tilde{\theta}(s)$ such that

$$
\begin{gathered}
\|g\|_{L_{\rho}^{2}}^{2}=\int_{\mathbb{R}^{N}}|g|^{2} e^{-\frac{|y|^{2}}{4}} d y, \\
\left\|w(., s)-\kappa e^{i \tilde{\theta}(s)}\right\|_{L_{\rho}^{2}}=\inf _{\theta \in[0,2 \pi]}\left\|w(., s)-\kappa e^{i \theta}\right\|_{L_{\rho}^{2}} \rightarrow 0 \text { as } s \rightarrow-\infty .
\end{gathered}
$$


We will slightly modify $\tilde{\theta}(s)$, so that if we define $v(y, s)$ by (3.9) for some $\theta(s)$ close to $\tilde{\theta}(s)$, then we have (i) of Lemma 2.4. We apply the implicit function theorem to $F: L_{\rho}^{2} \times \mathbb{R} \rightarrow \mathbb{R}$ defined by $F(w, \theta)=\int\left(\operatorname{Im}\left(w e^{-i \theta}-\kappa\right)-\delta \operatorname{Re}\left(w e^{-i \theta}-\kappa\right)\right) \rho$. Since we have $F\left(\kappa e^{i \tilde{\theta}}, \tilde{\theta}\right)=0$ and $\frac{\partial F}{\partial \theta}=-\int\left(\operatorname{Re}\left(w e^{-i \theta}\right)+\delta \operatorname{Im}\left(w e^{-i \theta}\right) \rho\right)$, hence $\frac{\partial F}{\partial \theta}\left(\kappa e^{i \tilde{\theta}}, \tilde{\theta}\right)=-\kappa \neq 0$, using the implicit function theorem and (3.11), we obtain the existence and uniqueness of $\mathcal{C}^{1} \theta(w)$ such that $F(w, \theta(w))=0$ and $\left|e^{i \theta(w)}-e^{i \tilde{\theta}}\right| \leq C_{0}\left\|w(., s)-\kappa e^{i \tilde{\theta}}\right\|_{L_{\rho}^{2}}$.

(ii) Since we have from (3.9),

$$
\begin{aligned}
\|v(.,)\|_{L_{\rho}^{2}}=\left\|w(., s)-\kappa e^{i \theta(s)}\right\|_{L_{\rho}^{2}} & \leq\left\|w(., s)-\kappa e^{i \tilde{\theta}}\right\|_{L_{\rho}^{2}}+\kappa\left|e^{i \theta}-e^{i \tilde{\theta}}\right| \\
& \leq\left(1+C_{0} \kappa\right)\left\|w(., s)-\kappa e^{i \tilde{\theta}(s)}\right\|_{L_{\rho}^{2}}
\end{aligned}
$$

using (3.11), we conclude that $\|v(., s)\|_{L_{\rho}^{2}} \rightarrow 0$ as $s \rightarrow-\infty$.

(iii) Writing $v=(1+i \delta) \tilde{v}_{1}+i \tilde{v}_{2}$, we rewrite (2.6) as follows:

$$
\begin{aligned}
& \tilde{v}_{1 s}=\mathcal{L} \tilde{v}_{1}+\theta^{\prime}(s)\left(\delta \tilde{v}_{1}+\tilde{v}_{2}\right)+\tilde{G}_{1}, \\
& \tilde{v}_{2 s}=(\mathcal{L}-1) \tilde{v}_{2}-\theta^{\prime}(s)\left(\left(1+\delta^{2}\right) \tilde{v}_{1}+\delta \tilde{v}_{2}+\kappa\right)+\tilde{G}_{2},
\end{aligned}
$$

where $\mathcal{L}$ is given in (3.3),

$$
\begin{aligned}
& \tilde{G}_{1}=\frac{p-\delta^{2}}{2 \kappa} \tilde{v}_{1}^{2}+\frac{1}{2 \kappa} \tilde{v}_{2}^{2}+O\left(|v|^{3}\right), \\
& \tilde{G}_{2}=\left(1+\delta^{2}\right) \frac{\tilde{v}_{1}\left(\delta \tilde{v}_{1}+\tilde{v}_{2}\right)}{\kappa}+O\left(|v|^{3}\right) .
\end{aligned}
$$

Note that $(1+i \delta) \tilde{G}_{1}+i \tilde{G}_{2}=G$ is defined in (2.7).

Now, we multiply (3.13) by $\rho$ and integrate over $\mathbb{R}$ to get

$$
\int \tilde{v}_{2 s} \rho=\int \operatorname{div}\left(\rho \nabla \tilde{v}_{2}\right)-\int \theta^{\prime}(s)\left(\left(1+\delta^{2}\right) \tilde{v}_{1}+\delta \tilde{v}+\kappa\right) \rho+\int \tilde{G}_{2} \rho .
$$

From (2.10), we have $\tilde{v}_{2}=\operatorname{Im}(v)-\delta \operatorname{Re}(v)$. We get from (i) of Lemma 2.4 that $\int \tilde{v}_{2 s} \rho=0$.

Since $\int \operatorname{div}\left(\rho \nabla \tilde{v}_{2}\right)=0$ we obtain

$$
\theta^{\prime}(s) \int\left(\left(1+\delta^{2}\right) \tilde{v}_{1}+\delta \tilde{v}_{2}+\kappa\right) \rho=\int \tilde{G}_{2} \rho
$$

Using (3.15), we have

$$
\left|\int \tilde{G}_{2} \rho\right| \leq C \int|v|^{2} \rho
$$

Recalling from (ii) that $\lim _{s \rightarrow-\infty}\|v\|=0$, we have

$$
\int\left(\left(1+\delta^{2}\right) \tilde{v}_{1}+\delta \tilde{v}_{2}+\kappa\right) \rho \rightarrow \int \kappa \rho \text { as } s \rightarrow-\infty .
$$

Thus, the conclusion follows from (3.16) and (3.17).

Step 2: Asymptotic behavior of $v$ as $s \rightarrow-\infty$.

First, we recall the decomposition (2.11):

$$
\begin{aligned}
v(y, s) & =(1+i \delta) \tilde{v}_{1}(y, s)+i \tilde{v}_{2}(y, s) \\
& =(1+i \delta) \sum_{m=0}^{\infty} \tilde{v}_{1 m}(s) h_{m}(y)+i \sum_{m=0}^{\infty} \tilde{v}_{2 m}(s) h_{m}(y),
\end{aligned}
$$


and introduce

$$
v_{-}(y, s)=(1+i \delta) \sum_{m=3}^{\infty} \tilde{v}_{1 m}(s) h_{m}(y)+i \sum_{m=1}^{\infty} \tilde{v}_{2 m}(s) h_{m}(y) .
$$

As we saw in Step 2 of Section 2, the modulation techniques give $\tilde{v}_{20}(s)=0$. Therefore, we have

$$
v(y, s)=(1+i \delta)\left(\tilde{v}_{10}(s) h_{0}(y)+\tilde{v}_{11}(s) h_{1}(y)+\tilde{v}_{12}(s) h_{2}(y)\right)+v_{-}(y, s) .
$$

Using ODE techniques, we are able to prove the following:

Proposition 3.8 (Classification of the behavior of $v(y, s)$ as $s \rightarrow-\infty$ ). As $s \rightarrow$ $-\infty$, one of the following situations occurs:

(i) $\left|\tilde{v}_{11}(s)\right|+\left|\tilde{v}_{12}(s)\right|+\left\|v_{-}(., s)\right\|_{L_{\rho}^{2}}=o\left(\tilde{v}_{10}(s)\right)$,

$\left\|v(., s)-(1+i \delta) C_{0} e^{s}\right\|_{L_{\rho}^{2}}=O\left(e^{\frac{3}{2} s}\right)$ and $\left|\theta^{\prime}(s)\right|=O\left(e^{2 s}\right)$ for some $C_{0} \in \mathbb{R}$.

(ii) $\left|\tilde{v}_{10}(s)\right|+\left|\tilde{v}_{12}(s)\right|+\left\|v_{-}(., s)\right\|_{L_{\rho}^{2}}=o\left(\tilde{v}_{11}(s)\right)$,

$\left\|v(., s)-(1+i \delta) C_{1} e^{s / 2} y\right\|_{L_{\rho}^{2}}=O\left(e^{(1-\varepsilon) s}\right)$ and $\left|\theta^{\prime}(s)\right|=O\left(e^{s}\right)$ for some $C_{1} \in \mathbb{R} \backslash$ 0 and $\varepsilon>0$.

(iii) $\left|\tilde{v}_{10}(s)\right|+\left|\tilde{v}_{11}(s)\right|+\left\|v_{-}(., s)\right\|_{L_{\rho}^{2}}=o\left(\tilde{v}_{12}(s)\right)$,

$\left\|v(., s)+(1+i \delta) \frac{\kappa}{4\left(p-\delta^{2}\right) s}\left(y^{2}-2\right)\right\|_{L_{\rho}^{2}}=O\left(\frac{\log |s|}{s^{2}}\right)$ and $\theta^{\prime}(s)=\frac{\left(1+\delta^{2}\right) \delta \kappa}{2\left(p-\delta^{2}\right)} \frac{1}{s^{2}}+$ $O\left(\frac{\log |s|}{s^{3}}\right)$.

Proof. As already pointed out by Filippas and Kohn on pp. 834-835 in FM95 in the case $\delta=0$, we can't use center manifold theory to get the result. In some sense, we are not able to say that the nonlinear terms in (3.12) and (3.13) are quadratic in the function space $L_{\rho}^{2}$. However, using ODE techniques similar to those of MZ98a and [FM95], we manage to conclude the proof. Since we add no real novelty, we leave the proof to Appendix $\mathrm{A}$.

Now, we recall Proposition 2.8 as it is a direct consequence of the proposition above.

Proposition 2.8. There exists $\theta_{0} \in \mathbb{R}$ such that $\theta(s) \rightarrow \theta_{0}$ and $\left\|w(., s)-\kappa e^{i \theta_{0}}\right\|_{L_{\rho}^{2}}$ $\rightarrow 0$ as $s \rightarrow-\infty$. More precisely, one of the following situations occurs as $s \rightarrow-\infty$, for some $C_{0} \in \mathbb{R}$ and $C_{1} \in \mathbb{R}^{*}$ :

$$
\begin{aligned}
& \text { (i) }\left\|w(., s)-\left\{\kappa+(1+i \delta) C_{0} e^{s}\right\} e^{i \theta_{0}}\right\|_{L_{\rho}^{2}} \leq C e^{\frac{3}{2} s}, \\
& \text { (ii) }\left\|w(., s)-e^{i \theta_{0}}\left\{\kappa-(1+i \delta) \frac{\kappa}{4\left(p-\delta^{2}\right) s}\left(y^{2}-2\right)-i \frac{\left(1+\delta^{2}\right) \delta \kappa^{2}}{2\left(p-\delta^{2}\right)^{2}} \frac{1}{s}\right\}\right\|_{L_{\rho}^{2}} \leq C \frac{\log |s|}{s^{2}} \text {, } \\
& \text { (iii) }\left\|w(., s)-\left\{\kappa+(1+i \delta) C_{1} e^{s / 2} y\right\} e^{i \theta_{0}}\right\|_{L_{\rho}^{2}} \leq C e^{(1-\varepsilon) s} .
\end{aligned}
$$

Proof of Proposition 2.8. From Proposition 3.8, we have $\|v(., s)\|_{L_{\rho}^{2}}=o(1 /|s|)$ in all cases. Then using (3.10), we obtain $\left|\theta^{\prime}(s)\right| \leq C / s^{2}$. Consequently, there exists a $\theta_{0}$ such that $\theta(s) \rightarrow \theta_{0}$ as $s \rightarrow-\infty$. Using the definition (3.9), we get the convergence for $w$.

We will just prove (ii), since the proofs for (i) and (iii) are the same and even easier.

Integrating the estimate for $\theta^{\prime}$ (see (iii) of Proposition 3.8), we get

$$
\theta(s)=\theta_{0}-\frac{\left(1+\delta^{2}\right) \delta \kappa}{2\left(p-\delta^{2}\right)^{2}} \frac{1}{s}+O\left(\frac{\log |s|}{s^{2}}\right)
$$


and

$$
e^{i \theta(s)}=e^{i \theta_{0}}\left\{1-i \frac{\left(1+\delta^{2}\right) \delta \kappa}{2\left(p-\delta^{2}\right)^{2} s}+O\left(\frac{\log |s|}{s^{2}}\right)\right\} .
$$

Using the fact that $w(y, s)=e^{i \theta(s)}(\kappa+v(y, s))$ (see(3.9)), the desired estimate follows from (3.20) and the $L_{\rho}^{2}$ expansion of $v$ from (ii) of Proposition 3.8. This concludes the proof of Proposition 2.8

\section{Step 3: Case where (i) of Proposition 2.8 holds.}

We prove Proposition 2.9. More precisely, we will prove that either $w \equiv \kappa e^{i \theta_{0}}$ or there exists $s_{0} \in \mathbb{R}$ such that $w=\varphi_{\delta}\left(s-s_{0}\right) e^{i \theta_{0}}$.

As we wrote in Step 3 of Section 2, if $\hat{\varphi}$ is defined by (2.17), then we have

$$
\forall s \leq \hat{s},\left\|w(., s)-\hat{\varphi}(s) e^{i \theta_{0}}\right\|_{L_{\rho}^{2}} \leq C e^{\frac{3}{2} s} .
$$

Our goal is to prove that $w \equiv \hat{\varphi}$ on $\mathbb{R} \times\left(-\infty, s_{*}\right]$. If we introduce $V=w-\hat{\varphi} e^{i \theta_{0}}$, then we see from (1.10) that $V$ satisfies:

$$
\partial_{s} V=(\tilde{\mathcal{L}}+l(s)) V+B
$$

where

$$
\tilde{\mathcal{L}} V=\Delta V-\frac{1}{2} y \cdot \nabla V+(1+i \delta) V,|l(s)| \leq C e^{s} \text { and }|B| \leq C|V|^{2} \text { for all } s \leq s_{1} .
$$

As we saw in Lemma 2.9 and (2.12), $\tilde{\mathcal{L}}$ is diagonal with respect to $\left(\tilde{V}_{1}, \tilde{V}_{2}\right)$ such that $V=(1+i \delta) \tilde{V}_{1}+i \tilde{V}_{2}$ and 1 is its largest eigenvalue.

Therefore, if we define $\|V\|_{\backsim}=\sqrt{\int\left(\tilde{V}_{1}^{2}+\tilde{V}_{2}^{2}\right) \rho}$, an equivalent norm to $\|V(., s)\|_{L_{\rho}^{2}}$, then we get from (3.22) and (3.23),

$$
\partial_{s}\|V\|_{\curvearrowleft} \leq\left(1+C e^{s}\right)\|V\|_{\curvearrowleft}+C\left\|V^{2}\right\|_{\curvearrowleft} .
$$

To estimate $\left\|V^{2}\right\|_{\curvearrowleft}$, it is easy to see from (3.22) and the fact that $V$ is bounded that

$$
\partial_{s}\left(\left|\tilde{V}_{1}\right|+\left|\tilde{V}_{2}\right|\right) \leq \Delta\left(\left|\tilde{V}_{1}\right|+\left|\tilde{V}_{2}\right|\right)-\frac{y}{2} \cdot \nabla\left(\left|\tilde{V}_{1}\right|+\left|\tilde{V}_{2}\right|\right)+C\left(\left|\tilde{V}_{1}\right|+\left|\tilde{V}_{2}\right|\right) .
$$

Therefore, we can apply the regularizing effect of Lemma 3.7 to $\left|\tilde{V}_{1}\right|+\left|\tilde{V}_{2}\right|$ and obtain the existence of $C^{*}>0$ and $s^{*}$, such that $\left\|V(., s)^{2}\right\|_{\backsim} \leq C^{*}\left\|V\left(., s-s^{*}\right)\right\|_{\curvearrowleft}^{2}$. Then, we obtain

$$
\forall s \leq s_{2}, I^{\prime}(s) \leq \frac{5}{4} I(s)+C I\left(s-s^{*}\right)^{2},
$$

where $I(s)=\|V(., s)\|_{\curvearrowleft}$. Since $I(s) \leq C e^{3 / 2 s}$ from (3.21), the following lemma from MZ98a allows us to conclude the proof.

Lemma 3.9. Consider $I(s)$ a positive $\mathcal{C}^{1}$ function such that (3.24) is satisfied and $0 \leq I(s) \leq C e^{3 / 2 s}$ for all $s \leq s_{2}$, for some $s_{2}$. Then, for some $s_{3} \leq s_{2}$, we have $I(s)=0$ for all $s \leq s_{3}$.

Proof. By a trivial induction, we prove that

$$
\forall n \in \mathbb{N}^{*} \text { and } s \leq s_{2} \quad I(s) \leq \frac{\left(C e^{3 / 4 s}\right)^{2^{n}}}{C} .
$$


Taking $s \leq s_{3}$, where $C e^{3 / 4 s_{3}}=1 / 2$ and making $n \rightarrow+\infty$, we see that for all $s \leq s_{3}, I(s)=0$.

Using Lemma 3.9, we see that $V \equiv 0$ on $\mathbb{R} \times\left(-\infty, s_{3}\right]$. Consequently, we have

$$
\forall(y, s) \in \mathbb{R} \times\left(-\infty, s_{3}\right], \quad w(y, s)=\hat{\varphi}(s) e^{i \theta_{0}} .
$$

From the uniqueness of the Cauchy problem for equation (1.10) and since $w$ is defined for all $(y, s) \in \mathbb{R} \times \mathbb{R}, \hat{\varphi}$ is also defined for $(y, s) \in \mathbb{R} \times \mathbb{R}$ and (3.25) holds for all $(y, s) \in \mathbb{R} \times \mathbb{R}$. Therefore, case (c) in (2.17) cannot hold and for all $(y, s) \in \mathbb{R}^{2}$, $w(y, s)=\kappa e^{i \theta_{0}}$ or $w(y, s)=\varphi_{\delta}\left(s-s_{0}\right) e^{i \theta_{0}}$. This concludes the proof of Proposition 2.9 and finishes Step 3.

Step 4: Irrelevance of case (iii) of Proposition 2.8.

As we said in Step 4 of Section 2, it is enough to prove Proposition 2.11 (which we recall here) to conclude this case:

Proposition 2.11. Assume that case (iii) from Proposition 2.8 holds. Then there exists $\varepsilon_{0}>0$, such that:

$$
\begin{array}{r}
\lim _{s \rightarrow-\infty} \sup _{|y| \leq \varepsilon_{0} \sqrt{-s}}\left|w(y, s)-G\left(\frac{y}{\sqrt{-s}}\right)\right|=0 \\
\text { where } G(\xi)=\kappa\left(1-\frac{(p-1)}{4\left(p-\delta^{2}\right)} \xi^{2}\right)^{-\frac{(1+i \delta)}{(p-1)}} .
\end{array}
$$

Indeed, let us first use Proposition 2.11 to find a contradiction ruling out case (iii) of Proposition 2.8, and then prove Proposition 2.11.

We define $u_{s_{0}}$ by

$$
u_{s_{0}}(\xi, \tau)=(1-\tau)^{-\frac{1+i \delta}{p-1}} w(y, s) \text {, where } y=\frac{\xi+\frac{\varepsilon_{0}}{2} \sqrt{-s_{0}}}{\sqrt{1-\tau}} \text { and } s=s_{0}-\log (1-\tau) \text {. }
$$

We note that $u_{s_{0}}$ is defined for all $\tau \in[0,1)$ and $\xi \in \mathbb{R} . u_{s_{0}}$ satisfies equation (1.2). The initial condition at time $\tau=0$ is $u_{s_{0}}(\xi, 0)=w\left(\xi+\frac{\varepsilon_{0}}{2} \sqrt{-s_{0}}, s_{0}\right)$. From (2.1), we have

$$
\forall \tau \in[0,1),\left\|u_{s_{0}}(., \tau)\right\|_{L^{\infty}} \leq M(1-\tau)^{-\frac{1}{p-1}} .
$$

Using Proposition 2.11, we get:

$$
\sup _{|\xi|<4\left|s_{0}\right|^{1 / 4}}\left|u_{s_{0}}(\xi, 0)-G\left(\frac{\varepsilon_{0}}{2}\right)\right| \equiv g\left(s_{0}\right) \rightarrow 0 \text { as } s_{0} \rightarrow-\infty .
$$

If we define $v$, the solution of:

$$
\left\{\begin{array}{l}
v^{\prime}=(1+i \delta)|v|^{p-1} v \\
v(0)=G\left(\frac{\varepsilon_{0}}{2}\right)
\end{array}\right.
$$

then $v(\tau)=\kappa\left(1-\frac{(p-1) \varepsilon_{0}^{2}}{16\left(p-\delta^{2}\right)}-\tau\right)^{-\frac{(1+i \delta)}{p-1}}$, which blows up at time $1-\frac{(p-1) \varepsilon_{0}^{2}}{16\left(p-\delta^{2}\right)}<1$. Therefore, there exists $\tau_{0}<1$, such that $\left|v\left(\tau_{0}\right)\right|=2 M\left(1-\tau_{0}\right)^{-\frac{1}{p-1}}$. Now, we consider the function $z=\left|\Re\left\{u_{s_{0}}-v\right\}\right|+\left|\Im\left\{u_{s_{0}}-v\right\}\right|$. Then we have for all $\tau \in\left[0, \tau_{0}\right]:$

$$
\partial_{\tau} z \leq \Delta z+C\left(\varepsilon_{0}\right) z
$$

We recall Lemma 2.11 (page 1063) from [MZ98b]. 
Lemma 3.10. Assume that $z(\xi, \tau)$ satisfies for all $|\xi| \leq 4 B_{1}$ and $\tau \in\left[0, \tau_{*}\right]$ :

$$
\left\{\begin{array}{l}
\partial_{\tau} z \\
z(\xi, 0) \leq \Delta z+\lambda z+\mu \\
\leq z_{0}, z(\xi, \tau) \leq B_{2}
\end{array}\right.
$$

where $\tau_{*} \leq 1$. Then, for all $|\xi| \leq B_{1}$ and $\tau \in\left[0, \tau_{*}\right]$,

$$
z(\xi, \tau) \leq e^{\lambda \tau}\left(z_{0}+\mu+C B_{2} e^{-B_{1}^{2} / 4}\right) .
$$

Using the fact that $z$ is bounded for all $\tau \in\left[0, \tau_{0}\right]$, by $B_{2}=B_{2}\left(\varepsilon_{0}\right)$ (use (3.28)), we apply this lemma with $B_{1}=\left|s_{0}\right|^{1 / 4}, \tau_{*}=\tau_{0}, z_{0}=g\left(s_{0}\right)$ and $\lambda=0$. Then, we get for all $\tau \in\left[0, \tau_{0}\right]$,

$$
\sup _{|\xi| \leq\left|s_{0}\right|^{1 / 4}} z(\xi, \tau) \leq g\left(s_{0}\right)+C\left(\varepsilon_{0}\right) e^{-\left|s_{0}\right|^{1 / 2} / 4} \rightarrow 0 \text { as } s_{0} \rightarrow-\infty .
$$

For $\left|s_{0}\right|$ large enough and $\xi=0$, we get $z\left(0, \tau_{0}\right) \leq \frac{M}{2}\left(1-\tau_{0}\right)^{-1 /(p-1)}$ and

$$
\left|u_{s_{0}}\left(0, \tau_{0}\right)\right| \geq\left|v\left(\tau_{0}\right)\right|-\left|z\left(0, \tau_{0}\right)\right| \geq \frac{3 M}{2}\left(1-\tau_{0}\right)^{-\frac{1}{p-1}},
$$

which is in contradiction with (3.28). Thus case (iii) of Proposition 2.8 cannot occur. It remains to prove Proposition 2.11

Proof of Proposition 2.11, If we note $f(y, s)=G\left(\frac{y}{\sqrt{-s}}\right)$. Then $f$ satisfies

$$
-\frac{y}{2} \cdot \nabla f-\frac{(1+i \delta)}{(p-1)} f+(1+i \delta)|f|^{p-1} f=0 .
$$

Consider some arbitrary $\varepsilon_{0} \in\left(0, R^{*}\right)$, where $R^{*}=\sqrt{\frac{4\left(p-\delta^{2}\right)}{(p-1)}}$. The parameter $\varepsilon_{0}$ will be fixed small enough later in the proof. If we note

$$
F(y, s)=f(y, s)+(1+i \delta) \frac{\kappa}{2\left(p-\delta^{2}\right)} \frac{1}{s}-i \frac{\left(1+\delta^{2}\right) \delta \kappa^{2}}{\left(p-\delta^{2}\right)^{2}} \frac{1}{s},
$$

then we see from (iii) of Proposition 2.8 that

$$
\left\|(F(., s)-w(., s))\left(1-\chi_{\varepsilon_{0}}\right)\right\|_{L_{\rho}^{2}}=O\left(\frac{\log |s|}{s^{2}}\right) \text { as } s \rightarrow-\infty,
$$

where

$$
\chi_{\varepsilon_{0}}(y, s)=1 \text { if } \frac{|y|}{\sqrt{|s|}} \geq 3 \varepsilon_{0} \text { and zero otherwise. }
$$

The formal idea of this proof is that $F$ solve in an approximate way the same equation as $w$ for $s \rightarrow-\infty$. By (3.31), $w$ and $F$ are very close in the region $|y| \sim 1$. Our task is to prove that they remain close in the larger region $|y| \leq \varepsilon_{0} \sqrt{-s}$, for some $\varepsilon_{0}$ to be chosen later. Let us consider a cutoff function

$$
\gamma(y, s)=\gamma_{0}\left(\frac{y}{\sqrt{-s}}\right)
$$

where $\gamma_{0} \in \mathcal{C}^{\infty}(\mathbb{R})$ is such that $\gamma_{0}(\xi)=1$ if $|\xi| \leq 3 \varepsilon_{0}$ and $\gamma_{0}(\xi)=0$ if $|\xi| \geq 4 \varepsilon_{0}$. We introduce

$$
\nu=(w-F)
$$


We note that $\nu=(1+i \delta) \tilde{\nu}_{1}+i \tilde{\nu}_{2}$ and $Z=\gamma\left(\left|\tilde{\nu}_{1}\right|+\left|\tilde{\nu}_{2}\right|\right)$. Our proof is the same as Velázquez Vel92], except for the fact that we need to perform a cutoff, since our profile $F(y, s)$ defined by (3.30) is singular on the parabola $y=R^{*} \sqrt{-s}$. The cutoff function will generate an extra term, which is difficult to handle. Let us present the major steps of the proof in the following. The proof of the presented lemmas will be given at the end of this step.

Lemma 3.11 (Estimates in modified $L_{\rho}^{2}$ spaces). There exists $\varepsilon_{0}>0$ such that the function $Z$ satisfies for all $s \leq s_{*}$ and $y \in \mathbb{R}$ :

$\partial_{s} Z-\Delta Z+\frac{1}{2} y \cdot \nabla Z-(1+\sigma) Z \leq C\left(Z^{2}+\frac{\left(y^{2}+1\right)}{s^{2}}+\chi_{\varepsilon_{0}}\right)-2 \operatorname{div}\left(\left(\left|\tilde{\nu}_{1}\right|+\left|\tilde{\nu}_{2}\right|\right) \nabla \gamma\right)$, where $s_{*} \in \mathbb{R}, \sigma=1 / 100$ and $\chi_{\varepsilon_{0}}$ is defined in (3.32). Moreover,

$$
N_{2 \varepsilon_{0} \sqrt{|s|}}^{2}(Z(s))=o(1) \text { as } s \rightarrow-\infty
$$

where the $\operatorname{norm} N_{r}^{q}(\psi)$ is defined, for all $r>0$ and $1 \leq q<\infty$, by

$$
N_{r}^{q}(\psi)=\sup _{|\xi| \leq r}\left(\int|\psi(y)|^{q} \exp \left(-\frac{(y-\xi)^{2}}{4}\right) d y\right)^{1 / q}
$$

Using the regularizing effect of the operator $\mathcal{L}$, we derive the following pointwise estimate, which allows us to conclude the proof of Proposition 2.11:

Lemma 3.12 (An upper bound for $Z(y, s)$ in $\left\{|y| \leq \varepsilon_{0} \sqrt{-s}\right\}$ ). We have

$$
\sup _{|y| \leq \varepsilon_{0} \sqrt{-s}} Z(y, s)=o(1) \text { as } s \rightarrow-\infty .
$$

Indeed, we have by definition of $Z$, for all $|y|<\varepsilon_{0} \sqrt{-s},|w-F|=\bar{\nu} \leq C Z(y, s)$. Thus, Proposition 2.11 follows from Lemma 3.12, It remains to prove Lemma 3.11 and Lemma 3.12 .

Proof of Lemma 3.11. The proof of (3.35) is straightforward and a bit technical. We leave it to Appendix B. Let us then prove (3.36). We take $s_{0}<s_{*}$ and $s_{0} \leq s<s_{*}$ such that $e^{\frac{s-s_{0}}{2}} \leq \sqrt{-s}$. We use the variation of constants formula in (3.35) to write

$$
\begin{aligned}
Z(y, s) \leq & S_{\sigma}\left(s-s_{0}\right) Z\left(., s_{0}\right) \\
& +\int_{s_{0}}^{s} S_{\sigma}(s-\tau)\left(C\left\{Z+\frac{\left(y^{2}+1\right)}{\tau^{2}}+\chi_{\varepsilon_{0}}\right\}-2 \operatorname{div}\left(\left(\left|\tilde{\nu}_{1}\right|+\left|\tilde{\nu}_{2}\right|\right) \nabla \gamma\right)\right) d \tau
\end{aligned}
$$

where $S_{\sigma}$ is the semigroup associated to the operator $\mathcal{L}_{\sigma} \phi=\Delta \phi-\frac{1}{2} y \cdot \nabla \phi+(1+\sigma) \phi$, defined on $L_{\rho}^{2}(\mathbb{R})$. The kernel of the semigroup $S_{\sigma}(\tau)$ is

$$
S_{\sigma}(\tau, y, z)=\frac{e^{(1+\sigma) \tau}}{\left(4 \pi\left(1-e^{-\tau}\right)\right)^{1 / 2}} \exp \left[-\frac{\left|y e^{-\tau / 2}-z\right|^{2}}{4\left(1-e^{-\tau}\right)}\right]
$$

Setting

$$
r \equiv r\left(s, s_{0}\right)=2 \varepsilon_{0} e^{\frac{s-s_{0}}{2}}=R_{1} e^{\frac{s-s_{0}}{2}}
$$


and taking the $N_{r}^{2}$-norm we obtain

$$
\begin{array}{r}
N_{r}^{2}(Z(., s)) \leq N_{r}^{2}\left(S_{\sigma}\left(s-s_{0}\right) Z\left(., s_{0}\right)\right)+C \int_{s_{0}}^{s} N_{r}^{2}\left(S_{\sigma}(s-\tau) Z(., \tau)^{2}\right) d \tau \\
+C \int_{s_{0}}^{s} N_{r}^{2}\left(S_{\sigma}(s-\tau)\left(\frac{\left(y^{2}+1\right)}{\tau^{2}}\right)\right) d \tau \\
+C \int_{s_{0}}^{s} N_{r}^{2}\left(S_{\sigma}(s-\tau) \chi_{\varepsilon_{0}}(y, \tau)\right) d \tau+C \int_{s_{0}}^{s} N_{r}^{2}\left(S_{\sigma}(s-\tau)\left(\operatorname{div}\left(\left(\left|\tilde{\nu}_{1}\right|+\left|\tilde{\nu}_{2}\right|\right) \nabla \gamma\right)\right) d \tau\right. \\
\equiv J_{1}+J_{2}+J_{3}+J_{4}+J_{5} .
\end{array}
$$

In comparison with [Vel92], we have a new term $J_{5}$ coming from the cutoff terms. Therefore, we just recall in the following claim the estimates on $J_{1}, \ldots J_{4}$ from Vel92, and treat $J_{5}$, which is a new ingredient in our proof.

Claim 3.13. We obtain as $s \rightarrow-\infty$,

$$
\begin{aligned}
\left|J_{1}\right| \leq & C e^{(1+\sigma)\left(s-s_{0}\right)} \frac{\log \left|s_{0}\right|}{\left|s_{0}^{2}\right|} \\
\left|J_{2}\right| \leq & C \int_{s_{0}}^{s_{0}+\left(\left(s-R_{0}\right)-s_{0}\right)_{+}} \frac{e^{(1+\sigma)\left(s-\tau-R_{0}\right)}}{\left(1-e^{s-\tau-R_{0}}\right)^{1 / 20}}\left(L_{r}^{2}\left(Z(., \tau)^{2}\right)\right) d \tau+C \frac{e^{\left(s-s_{0}\right)(1+\sigma)}}{s_{0}^{2}} \\
& \text { with } R_{0}=4 \varepsilon_{0}, \\
\left|J_{3}\right| \leq & C \frac{e^{\left(s-s_{0}\right)(1+\sigma)}}{s_{0}^{2}}\left(1+\left(s-s_{0}\right)\right) \\
\left|J_{4}\right| \leq & C e^{\left(s-s_{0}\right)(1+\sigma)} e^{\alpha s}, \text { where } \alpha>0 \\
\left|J_{5}\right| \leq & C e^{\left(s-s_{0}\right)(1+\sigma)} e^{\beta s}, \text { where } \beta>0
\end{aligned}
$$

Proof. See page 1578 in [Vel92] for $J_{1}, \ldots, J_{4}$.

Now, we treat $J_{5}$. We have from (3.38):

$$
\begin{aligned}
& S_{\sigma}(s-\tau)\left(-\operatorname{div}\left(\left(\left|\tilde{\nu}_{1}\right|+\left|\tilde{\nu}_{2}\right|\right) \nabla \gamma\right)\right) \\
& =-\frac{C e^{(s-\tau)(1+\sigma)}}{\left(1-e^{s-\tau}\right)^{1 / 2}} \int_{\mathbb{R}} \exp \left(-\frac{\left(y e^{-(s-\tau) / 2}-\lambda\right)^{2}}{4\left(1-e^{-(s-\tau)}\right)}\right) \operatorname{div}\left(\left(\left|\tilde{\nu}_{1}\right|+\left|\tilde{\nu}_{2}\right|\right) \nabla \gamma\right) d \lambda \\
& =\frac{C e^{(s-\tau)(1+\sigma)}}{\left(1-e^{s-\tau}\right)^{1 / 2}} \int_{\mathbb{R}}-\frac{\left(y e^{-(s-\tau) / 2}-\lambda\right)}{2\left(1-e^{-(s-\tau)}\right)} \exp \left(-\frac{\left(y e^{-(s-\tau) / 2}-\lambda\right)^{2}}{4\left(1-e^{-(s-\tau)}\right)}\right)\left(\left|\tilde{\nu}_{1}\right|+\left|\tilde{\nu}_{2}\right|\right) \nabla \gamma d \lambda .
\end{aligned}
$$

Since $w$ and $F$ are bounded for $\frac{|y|}{\sqrt{-\tau}} \leq \frac{R^{*}}{2}$ and $\operatorname{supp}(\nabla \gamma) \subset\left(-4 \varepsilon_{0} \sqrt{-\tau},-3 \varepsilon_{0} \sqrt{-\tau}\right)$ $\cup\left(3 \varepsilon_{0} \sqrt{-\tau}, 4 \varepsilon_{0} \sqrt{-\tau}\right)$, we have

$$
\begin{aligned}
\left|\left(\left|\tilde{\nu}_{1}\right|+\left|\tilde{\nu}_{2}\right|\right) \nabla \gamma\right| & \leq C\left(\left|\tilde{\nu}_{1}\right|+\left|\tilde{\nu}_{2}\right|\right) \mathbb{I}_{\left\{3 \varepsilon_{0} \leq \frac{|y|}{\sqrt{-\tau}} \leq 4 \varepsilon_{0}\right\}} \\
& \leq C\left(\mathbb{I}_{3 \varepsilon_{0} \leq \frac{|y|}{\sqrt{-\tau}} \leq 4 \varepsilon_{0}}\right) \leq C \chi_{\varepsilon_{0}} .
\end{aligned}
$$

Using the Cauchy-Schwarz inequality, we obtain

$$
\left|S_{\sigma}(s-\tau)\left(-\operatorname{div}\left(\left(\left|\tilde{\nu}_{1}\right|+\left|\tilde{\nu}_{2}\right|\right) \nabla \gamma\right)\right)\right| \leq \frac{C e^{(s-\tau)(1+\sigma)}}{\left(1-e^{s-\tau}\right)^{3 / 2}} \mathcal{I}_{1} \mathcal{I}_{2},
$$


where

$$
\begin{aligned}
& \mathcal{I}_{1}=\left(\int_{\mathbb{R}}\left(y e^{-(s-\tau) / 2}-\lambda\right)^{2} \exp \left(-\frac{\left(y e^{-(s-\tau) / 2}-\lambda\right)^{2}}{4\left(1-e^{-(s-\tau)}\right)}\right) d \lambda\right)^{1 / 2}, \\
& \mathcal{I}_{2}=\left(\int_{\mathbb{R}} \exp \left(-\frac{\left(y e^{-(s-\tau) / 2}-\lambda\right)^{2}}{4\left(1-e^{-(s-\tau)}\right)}\right) \chi_{\varepsilon_{0}} d \lambda\right)^{1 / 2} .
\end{aligned}
$$

Doing a change of variables, we obtain $\mathcal{I}_{1}=C\left(1-e^{-(s-\tau)}\right)^{3 / 4}$. Furthermore, we have

$$
\mathcal{I}_{2}^{2} \leq \mathcal{I}_{3}\left(\int_{\mathbb{R}} \chi_{\varepsilon_{0}} e^{-\frac{\lambda^{2}}{4}} d \lambda\right)^{1 / 2}
$$

where

$$
\mathcal{I}_{3}=\left(\int \exp \left(-\frac{\left(y e^{-(s-\tau) / 2}-\lambda\right)^{2}}{2\left(1-e^{-(s-\tau)}\right)}+\frac{\lambda^{2}}{4}\right) d \lambda\right)^{1 / 2} .
$$

We introduce $\theta=y e^{-(s-\tau) / 2}$ and, by completing squares, we readily check that

$$
\frac{\lambda^{2}}{4}-\frac{(\theta-\lambda)^{2}}{2\left(1-e^{-(s-\tau)}\right)}=-\frac{\left(1+e^{-(s-\tau)}\right)}{4\left(1-e^{-(s-\tau)}\right)}\left(\lambda-\frac{2 \theta}{\left(1+e^{-(s-\tau)}\right)}\right)^{2}+\frac{\theta^{2}}{2\left(1+e^{-(s-\tau)}\right)} .
$$

Then we obtain

$$
\mathcal{I}_{3}^{2}=C\left(\frac{\left(1-e^{-(s-\tau)}\right)}{\left(1+e^{-(s-\tau)}\right)}\right)^{1 / 2} \exp \left(\frac{\theta^{2}}{2\left(1-e^{-(s-\tau)}\right)}\right) .
$$

Therefore,

$$
\left|N_{r}^{2}\left(S_{\sigma}(s-\tau) \operatorname{div}\left(\left(\left|\tilde{\nu}_{1}\right|+\left|\tilde{\nu}_{2}\right|\right) \nabla \gamma\right)\right)\right| \leq C \frac{e^{(s-\tau)(1+\sigma)}}{\left(1+e^{-(s-\tau)}\right)^{1 / 8}\left(1-e^{-(s-\tau)}\right)^{5 / 8}}\left\|\chi_{\varepsilon_{0}}\right\|^{1 / 2} \mathcal{I}_{4},
$$

where

$$
\mathcal{I}_{4}=N_{r}^{2}\left(\exp \left(\frac{y^{2} e^{-(s-\tau)}}{8\left(\left(1-e^{-(s-\tau)}\right)\right)}\right)\right)
$$

Let us compute $\mathcal{I}_{4}$. Using the fact that

$$
\begin{aligned}
& -\frac{(y-\mu)^{2}}{4}+\frac{y^{2} e^{-(s-\tau)}}{4\left(1-e^{-(s-\tau)}\right)} \\
& =\frac{1}{4}\left(-\left(y\left(1+e^{-(s-\tau)}\right)^{-1 / 2}-\mu\left(1+e^{-(s-\tau)}\right)^{1 / 2}\right)^{2}+\mu^{2} e^{-(s-\tau)}\right),
\end{aligned}
$$

and doing a change of variables, we obtain

$$
\begin{aligned}
& \int_{\mathbb{R}} \exp \left(-\frac{(y-\mu)^{2}}{4}+\frac{y^{2} e^{-(s-\tau)}}{4\left(1-e^{(s-\tau)}\right)}\right) d y \\
& \leq C \exp \left(\frac{\mu^{2} e^{-(s-\tau)}}{4}\right) \int_{\mathbb{R}} \exp \left(-\frac{1}{4}\left(y\left(1+e^{-(s-\tau)}\right)^{-1 / 2}-\mu\left(1+e^{-(s-\tau)}\right)^{1 / 2}\right)^{2}\right) d y .
\end{aligned}
$$

Hence $\mathcal{I}_{4} \leq C\left(1+e^{-(s-\tau)}\right)^{1 / 8}$ and

$$
N_{r}^{2}\left(S_{\sigma}(s-\tau) \operatorname{div}\left(\left(\left|\tilde{\nu}_{1}\right|+\left|\tilde{\nu}_{2}\right|\right) \nabla \gamma\right)\right) \leq C \frac{e^{(s-\tau)(1+\sigma)}}{\left(1-e^{-(s-\tau)}\right)^{5 / 8}}\left(\int_{|\lambda| \geq R_{1} \sqrt{-\tau}} e^{-\frac{\lambda^{2}}{4}} d \lambda\right) .
$$


This gives

$$
\left|J_{5}\right|=\int_{s_{0}}^{s} N_{r}^{2}\left(S_{\sigma}(s-\tau)\left(\operatorname{div}\left(\left|\tilde{\nu}_{1}\right|+\left|\tilde{\nu}_{2}\right|\right)\right)\right) d \tau \leq C(\eta) e^{\left(s-s_{0}\right)(1+\sigma)} e^{\alpha s_{0}},
$$

where $\alpha>0$. This concludes the proof of the claim 3.13 ,

Summing up $J_{i=1 . .5}$, from claim 3.13 we obtain

$$
\begin{aligned}
& N_{r}^{2}(Z(., s)) \leq \\
& e^{\left(s-s_{0}\right)(1+\sigma)} C \frac{\log \left|s_{0}\right|}{s_{0}^{2}}+C \int_{s_{0}}^{s_{0}+\left(\left(s-R_{0}\right)-s_{0}\right)+} \frac{e^{\left(s-\tau-R_{0}\right)(1+\sigma)}}{\left(1-e^{s-\tau-R_{0}}\right)^{1 / 20}}\left(N_{r}^{2}(Z(., \tau))\right)^{2} d \tau .
\end{aligned}
$$

Now, we recall the following from Vel92]:

Lemma 3.14. Let $\varepsilon, C, R, \sigma$ and $\alpha$ be positive constants, $0<\alpha<1$ and assume that $H(s)$ is a family of continuous functions satisfying:

$$
H(s) \leq \varepsilon e^{s(1+\sigma)}+C \int_{0}^{(s-R)_{+}} \frac{e^{(s-\tau)(1+\sigma)} H(\tau)^{2}}{\left(1-e^{(s-\tau-R)}\right)^{\alpha}} d \tau \text { for } s>0 .
$$

Then there exists $\xi=\xi(R, C, \alpha)$ such that for any $\varepsilon \in\left(0, \varepsilon_{1}\right)$ and any $s$ for which $\varepsilon e^{s(1+\sigma)} \leq \xi$, we have

$$
H(s) \leq 2 \varepsilon e^{s(1+\sigma)} .
$$

Proof. See the proof of Lemma 2.2 from Vel92]. Note that the proof of [Vel92] is done in the case $\sigma=0$, but it can be adapted to some $\sigma>0$ with no difficulty.

We conclude that $N_{r\left(\tau, s_{0}\right)}^{2}(Z(., s)) \leq C e^{\left(s-s_{0}\right)(1+\sigma) \frac{\log \left|s_{0}\right|}{s_{0}^{2}}}$ as $s \rightarrow-\infty$. If we fix $s=-e^{\left(s-s_{0}\right)}$, then we obtain $s \sim s_{0}, \log |s| \sim \log \left|s_{0}\right|$ and $N_{R_{1} \sqrt{-s}}^{2}(Z(., s)) \leq$ $C s^{1+\sigma} \frac{\log \left|s_{0}\right|}{s_{0}^{2}} \leq C \frac{\log |s|}{s^{1-\sigma}} \rightarrow 0$ as $s \rightarrow-\infty$. Since $\sigma=\frac{1}{100}$, we get $N_{R_{1} \sqrt{-s}}^{2}(Z(., s))=$ $o(1)$, as $s \rightarrow-\infty$.

This concludes the proof of Lemma 3.11 .

Proof of Lemma 3.12. We aim at bounding $Z(y, s)$ for $|y| \leq R_{2} \sqrt{-s}$ in terms of $N_{R_{1} \sqrt{-s^{\prime}}}\left(Z\left(s^{\prime}\right)\right)$, where $R_{2}=\varepsilon_{0}$ and $R_{1}=2 \varepsilon_{0}$, for some $s^{\prime}<s$. Starting from equation (3.35), we do as in Vel92]:

$$
\begin{aligned}
Z(., s) \leq & \left\{e^{C R_{0}} S\left(R_{0}\right) Z\left(., s-R_{0}\right)\right\} \\
& +\left\{C \int_{s-R_{0}}^{s} e^{C(s-\tau)} S(s-\tau)\left(\frac{\left(y^{2}+1\right)}{\tau^{2}}+\chi_{\varepsilon_{0}}\right) d \tau\right\} \\
& -\left\{2 \int_{s-R_{0}}^{s} e^{C(s-\tau)} S(s-\tau)\left(\operatorname{div}\left(\left(\left|\tilde{\nu}_{1}\right|+\left|\tilde{\nu}_{2}\right|\right) \nabla \gamma\right)\right) d \tau\right\} \\
= & \mathcal{M}_{1}+\mathcal{M}_{2}+\mathcal{M}_{3}, \text { where } R_{0}=4 \varepsilon_{0},
\end{aligned}
$$

where $S$ is the semigroup associated to the operator $\mathcal{L}$ defined in (3.3). The terms $\mathcal{M}_{1}$ and $\mathcal{M}_{2}$ are estimated in the following:

Claim 3.15 (Velázquez). There exists $s_{0}$, such that for all $s \leq s_{0}$,

$$
\begin{aligned}
& \sup _{|y| \leq R_{2} \sqrt{-s}}\left|\mathcal{M}_{1}\right|=\sup _{|y| \leq R_{2} \sqrt{-s}} \int_{s-R_{0}}^{s}\left(\frac{|y|^{2}+1}{s^{2}}+\chi_{\varepsilon_{0}}\right) \leq \frac{C}{|s|}, \\
& \sup _{|y| \leq R_{2} \sqrt{-s}}\left|\mathcal{M}_{2}\right|= \\
& \sup _{|y| \leq R_{2} \sqrt{-s}}\left|e^{C R_{0}} S\left(R_{0}\right) Z\left(., s-R_{0}\right)\right|=o(1) \text { as } s \rightarrow-\infty .
\end{aligned}
$$


Proof. See page 1581 from Vel92 and Lemma 6.5 in HV93 in a similar case.

It remains to estimate $\mathcal{M}_{3}$. Using the same calculus in (3.40), we write

$$
\begin{aligned}
& \left|S(s-\tau)\left(-\operatorname{div}\left(\left(\left|\tilde{\nu}_{1}\right|+\left|\tilde{\nu}_{2}\right|\right) \nabla \gamma\right)\right)\right| \\
& =\left|\frac{C e^{s-\tau}}{\left(1-e^{s-\tau}\right)^{1 / 2}} \int_{\mathbb{R}} \exp \left(-\frac{\left(y e^{-(s-\tau) / 2}-\lambda\right)^{2}}{4\left(1-e^{-(s-\tau)}\right)}\right) \operatorname{div}\left(\left(\left|\tilde{\nu}_{1}\right|+\left|\tilde{\nu}_{2}\right|\right) \nabla \gamma\right) d \lambda\right| \\
& =\left|\frac{C e^{s-\tau}}{\left(1-e^{s-\tau}\right)^{1 / 2}} \int_{\mathbb{R}}-\frac{\left(y e^{-(s-\tau) / 2}-\lambda\right)}{2\left(1-e^{-(s-\tau)}\right)} \exp \left(-\frac{\left(y e^{-(s-\tau) / 2}-\lambda\right)^{2}}{4\left(1-e^{-(s-\tau)}\right)}\right)\left(\left|\tilde{\nu}_{1}\right|+\left|\tilde{\nu}_{2}\right|\right) \nabla \gamma d \lambda\right| \\
& \leq \frac{C e^{s-\tau}}{\left(1-e^{s-\tau}\right)^{3 / 2}} \int_{\mathbb{R}}\left|y e^{-(s-\tau) / 2}-\lambda\right| \exp \left(-\frac{\left(y e^{-(s-\tau) / 2}-\lambda\right)^{2}}{4\left(1-e^{-(s-\tau)}\right)}\right) \chi_{\varepsilon_{0}} d \lambda \\
& \leq \frac{C e^{s-\tau} \sqrt{-\tau}}{\left(1-e^{s-\tau}\right)^{3 / 2}} \int_{\mathbb{R}} \exp \left(-\frac{\left(y e^{-(s-\tau) / 2}-\lambda\right)^{2}}{4\left(1-e^{-(s-\tau)}\right)}\right) \chi_{\varepsilon_{0}} d \lambda .
\end{aligned}
$$

We make the change of variables $z=\left(1-e^{-(s-\tau)}\right)^{-1 / 2}\left(\lambda-e^{-(\tau-s) / 2} y\right)$ and we obtain

$$
\int_{\mathbb{R}} \exp \left(-\frac{\left(y e^{-(s-\tau) / 2}-\lambda\right)^{2}}{4\left(1-e^{-(s-\tau)}\right)}\right) \chi_{\varepsilon_{0}} d \lambda \leq\left(1-e^{s-\tau}\right)^{1 / 2} \int_{\Sigma} e^{-z^{2} / 4} d z
$$

where

$$
\Sigma=\left\{z \in \mathbb{R}:\left|z+e^{-(\tau-s) / 2}\left(1-e^{s-\tau}\right)^{-1 / 2} y\right| \geq 3 \varepsilon_{0}\left(1-e^{s-\tau}\right)^{-1 / 2} \sqrt{-\tau}\right\} .
$$

Since $\left|y e^{-(\tau-s) / 2}\right| \leq \varepsilon_{0} \sqrt{-s}$, we readily see that $\Sigma \subset\left\{z \in \mathbb{R}:|z| \geq \varepsilon_{0} \sqrt{-s}\right\}$. Then we conclude that

$$
\left|S(s-\tau)\left(-\operatorname{div}\left(\left(\left|\tilde{\nu}_{1}\right|+\left|\tilde{\nu}_{2}\right|\right) \nabla \gamma\right)\right)\right| \leq \frac{C e^{s-\tau}}{\left(1-e^{s-\tau}\right)} e^{\beta s}, \text { where } \beta>0,
$$

and we obtain

$$
\sup _{|y| \leq R_{2} \sqrt{-s}}\left|\mathcal{M}_{3}\right|=o\left(\frac{1}{|s|}\right) \text { as } s \rightarrow-\infty .
$$

Putting together $\mathcal{M}_{i=1, \ldots, 3}$, the proof of Lemma 3.12 is complete.

This concludes also the proof of Proposition 2.11 and rules out case (iii) of Proposition 2.8.

\section{Step 5: Irrelevance of case (ii) of Proposition 2.8 .}

To conclude the proof of Theorem 10 we consider case (ii) of Proposition 2.8. We assume as in the previous case that $\theta_{0}=0$. We claim that the following proposition allows us to reach a contradiction in this case.

Proposition 3.16. There exists $\varepsilon_{0}>0$ such that

$$
\lim _{s \rightarrow-\infty} \sup _{|y| \leq \varepsilon_{0} e^{-s / 2}}\left|w(y, s)-G\left(y e^{s / 2}\right)\right|=0 \text {, where } G(\xi)=\kappa\left(1-C_{1} \kappa^{-p} \xi\right)^{-\frac{(1+i \delta)}{(p-1)}} .
$$

Indeed, as in the previous step, first, we will find a contradiction ruling out case (ii) of Proposition 2.8 and then prove Proposition 3.16 .

We define $u_{s_{0}}$ by

$$
u_{s_{0}}(\xi, \tau)=(1-\tau)^{-\frac{1+i \delta}{p-1}} w(y, s), \text { where } y=\frac{\xi+\frac{\varepsilon_{0}}{2} e^{-s_{0} / 2}}{\sqrt{1-\tau}} \text { and } s=s_{0}-\log (1-\tau) .
$$


$u_{s_{0}}$ is defined for all $\tau \in[0,1)$ and $\xi \in \mathbb{R}$. $u_{s_{0}}$ satisfies equation (1.2). The initial condition at time $\tau=0$ is $u_{s_{0}}(\xi, 0)=w\left(\xi+\frac{\varepsilon_{0}}{2} e^{-s_{0} / 2}, s_{0}\right)$. From (2.1), we have

$$
\forall \tau \in[0,1),\left\|u_{s_{0}}(., \tau)\right\|_{L^{\infty}} \leq M(1-\tau)^{-\frac{1}{p-1}} .
$$

Using Proposition 3.16, we get

$$
\sup _{|\xi|<4 e^{-s_{0} / 4}}\left|u_{s_{0}}(\xi, 0)-G\left(\varepsilon_{0} / 2\right)\right| \equiv g\left(s_{0}\right) \rightarrow 0 \text { as } s_{0} \rightarrow-\infty .
$$

If we define $v$, the solution of

$$
\left\{\begin{array}{l}
v^{\prime}=(1+i \delta)|v|^{p-1} v \\
v(0)=G\left(\frac{\varepsilon_{0}}{2}\right)
\end{array}\right.
$$

then $v(\tau)=\kappa\left(1-C_{1} \kappa^{-p \frac{\varepsilon_{0}}{2}}-\tau\right)^{-\frac{(1+i \delta)}{p-1}}$, which blows up at time $1-C_{1} \kappa^{-p \frac{\varepsilon_{0}}{2}}<1$. Therefore, there exists $\tau_{0}<1$, such that $\left|v\left(\tau_{0}\right)\right|=2 M\left(1-\tau_{0}\right)^{-\frac{1}{p-1}}$. Now, we consider the function $z=\left|\Re\left\{u_{s_{0}}-v\right\}\right|+\left|\Im\left\{u_{s_{0}}-v\right\}\right|$. Then we have for all $\tau \in\left[0, \tau_{0}\right]:$

$$
\partial_{\tau} z \leq \Delta z+C\left(\varepsilon_{0}\right) z .
$$

Using the fact that $z$ is bounded for all $\tau \in\left[0, \tau_{0}\right]$ by $B_{2}=B_{2}\left(\varepsilon_{0}\right)$ (use (3.44)), we use Lemma 3.10 with $B_{1}=e^{-s_{0} / 4}, \tau_{*}=\tau_{0}, z_{0}=g\left(s_{0}\right)$ and $\lambda=0$. We obtain for all $\tau \in\left[0, \tau_{0}\right]$,

$$
\sup _{|\xi| \leq e^{-\varepsilon_{0} / 4}}|z(\xi, \tau)| \leq g\left(s_{0}\right)+C\left(\varepsilon_{0}\right) e^{-e^{-\mid s_{0} / / 2} / 4} \rightarrow 0 \text { as } s_{0} \rightarrow-\infty .
$$

For $\left|s_{0}\right|$ large enough and $\xi=0$, we get $\left|z\left(0, \tau_{0}\right)\right| \leq(M / 2)\left(1-\tau_{0}\right)^{-\frac{1}{p-1}}$ and

$$
\left|u_{s_{0}}\left(0, \tau_{0}\right)\right| \geq \frac{3}{2} M\left(1-\tau_{0}\right)^{-\frac{1}{p-1}},
$$

which by (3.43) is in contradiction with (3.44) and case (ii) of Proposition 2.8 is ruled out. Now, we prove Proposition 3.16

Proof of Proposition 3.16. The proof is very similar to that of Proposition 2.11. We note that $f(y, s)=G\left(y e^{s / 2}\right)$. Then $f$ satisfies

$$
-\partial_{s} f-\frac{1}{2} y \cdot \nabla f-(1+i \delta) \frac{f}{(p-1)}+(1+i \delta)|f|^{p-1} f=0 .
$$

Consider an arbitrary $\varepsilon_{0} \in\left(0, \frac{R^{*}}{10}\right)$, where $R^{*}=\frac{\kappa^{p}}{C_{1}} \cdot \varepsilon_{0}$ will be fixed small enough later. Let us consider a cutoff function $\gamma(y, s)=\gamma_{0}\left(y e^{s / 2}\right)$, where $\gamma_{0} \in \mathcal{C}^{\infty}(\mathbb{R})$ such that $\gamma_{0}(\xi)=1$ if $|\xi| \leq 3 \varepsilon_{0}$ and $\gamma_{0}(\xi)=0$ if $|\xi| \geq 4 \varepsilon_{0}$. We note $\nu=(w-f)$ and $Z=\gamma\left(\left|\tilde{\nu}_{1}\right|+\left|\tilde{\nu}_{2}\right|\right)$. From (ii) of Proposition 2.8, we have

$$
\|Z\| \leq C e^{s(1-\varepsilon)} \text { as } s \rightarrow-\infty, \text { for some } \varepsilon>0 .
$$

As in the previous case, we divide our proof into two parts given in the following lemmas.

Lemma 3.17 (Estimates in the modified $L_{\rho}^{2}$ spaces). There exists $\varepsilon_{0}>0$ such that the function $Z$ satisfies for all $s \leq s_{*}$ and $y \in \mathbb{R}$,

$$
\partial_{s} Z-\Delta Z+\frac{1}{2} y \cdot \nabla Z-(1+\sigma) Z \leq C\left(Z^{2}+e^{s}+\chi_{\varepsilon_{0}}\right)-2 \operatorname{div}\left(\left(\left|\tilde{\nu}_{1}\right|+\left|\tilde{\nu}_{2}\right|\right) \nabla \gamma\right)
$$


where $s_{*} \in \mathbb{R}, \sigma=\frac{1}{100}$ and

$$
\chi_{\varepsilon_{0}}(y, s)=1 \text { if }|y| e^{s / 2} \geq 3 \varepsilon_{0} \text { and zero otherwise. }
$$

Moreover, we have

$$
N_{2 \varepsilon_{0} e^{-s / 2}}^{2}(Z(s))=o(1) \text { as } s \rightarrow-\infty .
$$

As in Step 4, the following lemma allows us to conclude the proof of Proposition 3.16 .

Lemma 3.18 (An upper bound for $Z(y, s)$ in $|y| \leq \varepsilon_{0} e^{-s / 2}$ ). We have

$$
\sup _{|y| \leq \varepsilon_{0} e^{-s / 2}} Z(y, s)=o(1) \text { as } s \rightarrow-\infty .
$$

It remains to prove Lemmas 3.17 and 3.18 to conclude the proof of Proposition 3.16. Here, we only sketch the proof of Lemma 3.17, since it is completely similar to Step 4. We don't give the proof of Lemma 3.18. We refer the reader to Step 4 and Proposition 2.4 from Velázquez [Vel92] for similar situations.

Proof of Lemma 3.17. As in the previous step, we leave the proof of (3.48) to Appendix B.

Let us now apply the variation of constants formula and take the norm $N_{r\left(s, s_{0}\right)}^{2}$, where $r\left(s, s_{0}\right)$ is as in (3.39). Assume that $s_{0}<2 s_{*}$. Then for all $s_{0} \leq s \leq \frac{s_{0}}{2}$, we have

$$
\begin{aligned}
N_{r}^{2}(Z(., s)) \leq & N_{r}^{2}\left(S_{\sigma}\left(s-s_{0}\right) Z\left(., s_{0}\right)\right)+C \int_{s_{0}}^{s} N_{r}^{2}\left(S_{\sigma}(s-\tau)\left(Z(., \tau)^{2}\right)\right) d \tau \\
& +C \int_{s_{0}}^{s} N_{r}^{2}\left(S(s-\tau)\left(e^{\tau}\right)\right) d \tau+C \int_{s_{0}}^{s} N_{r}^{2}\left(S_{\sigma}(s-\tau)\left(\chi_{\varepsilon_{0}}(., \tau)\right)\right) d \tau \\
& -2 \int_{s_{0}}^{s} N_{r}^{2}\left(S_{\sigma}(s-\tau)\left(\operatorname{div}\left(\left(\left|\tilde{\nu}_{1}\right|+\left|\tilde{\nu}_{2}\right|\right) \nabla \gamma\right)\right)\right) d \tau \\
= & J_{1}+J_{2}+J_{3}+J_{4}+J_{5} .
\end{aligned}
$$

Arguing as in Step 4 and using (3.47), we prove:

Claim 3.19.

$$
\begin{aligned}
&\left|J_{1}\right| \leq C e^{\left(s-s_{0}\right)(1+\sigma)} e^{s_{0}(1-\varepsilon)} \\
&\left|J_{2}\right| \leq C \int_{s_{0}}^{s_{0}+\left(\left(s-R_{0}\right)-s_{0}\right)+} \frac{e^{\left(s-\tau-R_{0}\right)(1+\sigma)}}{\left(1-e^{s-\tau-R_{0}}\right)^{1 / 20}}\left(L_{r}^{2}\left(Z(., s)^{2}\right)\right) d \tau \\
& \quad+C e^{\left(s-s_{0}\right)(1+\sigma)} e^{s} \text { with } R_{0}=4 \varepsilon_{0} \\
&\left|J_{3}\right| \leq C e^{\left(s-s_{0}\right)(1+\sigma)} e^{s}, \\
&\left|J_{4}\right| \leq C e^{\left(s-s_{0}\right)(1+\sigma)} e^{-\alpha e^{-s}} \text { where } \alpha>0 \\
&\left|J_{5}\right| \leq C e^{\left(s-s_{0}\right)(1+\sigma)} e^{-\beta e^{-s}} \text { where } \beta>0 .
\end{aligned}
$$

Proof. To estimate $J_{i=1 . .4}$, see page 1584 in Vel92. To treat $J_{5}$, we proceed as in the proof of Lemma 3.11 of the previous step.

Summing up $J_{i=1, \ldots, 5}$, we obtain

$$
\begin{aligned}
& N_{r}^{2}(Z(., s)) \\
& \leq C e^{\left(s-s_{0}\right)(1+\sigma)} e^{(1-\varepsilon) s}+C \int_{s_{0}}^{s_{0}+\left(\left(s-R_{0}\right)-s_{0}\right)_{+}} \frac{e^{\left(s-\tau-R_{0}\right)(1+\sigma)}}{\left(1-e^{\left.s-\tau-R_{0}\right)^{1 / 20}}\right.}\left(L_{r}^{2}\left(Z(., s)^{2}\right)\right) d \tau .
\end{aligned}
$$

Then using Proposition 3.14, we get $N_{r\left(s, s_{0}\right)}^{2}(Z(., s)) \leq C e^{\left(s-s_{0}\right)(1+\sigma)} e^{(1-\varepsilon) s}$ as $s \rightarrow$ $-\infty$ for $s_{0} \leq s \leq \frac{s_{0}}{2}$. If we fix $s=s_{0} / 2$, then we obtain $N_{r\left(s, s_{0}\right)}^{2}(Z(., s)) \leq$ 
$C e^{s(2(1-\varepsilon)-(1+\sigma))} \leq C e^{s(1-(2 \varepsilon+\sigma))} \rightarrow 0$ as $s \rightarrow-\infty$, since $\epsilon$ is small enough and $\sigma=\frac{1}{100}$. This concludes the proof of Lemma 3.17 .

As mentioned earlier, we don't give the proof of Lemma 3.18 and we refer the reader to Step 4 and Section 2 from [Vel92]. This concludes the proof of Proposition 3.16 and rules out case (ii) of Proposition 2.8.

Conclusion of Part 3 and the sketch of proof of the Liouville theorem: As we wrote in Section 2, we conclude from Steps 4 and 5 that cases (ii) and (iii) of Proposition 2.8 are ruled out. By Step 3, we obtain that $w \equiv \kappa e^{i \theta_{0}}$ or $w \equiv \varphi_{\delta}\left(s-s_{0}\right) e^{i \theta_{0}}$ for some real $s_{0}$ and $\theta_{0}$, where $\varphi_{\delta}$ is defined in Theorem 1, which is the desired conclusion of Theorem 1 .

\section{Applications of the Liouville theorem for a type I BlOW-UP SOLUTION OF (1.2)}

In this section we describe how to adapt to the case $\delta \neq 0$ the proof of Proposition 3 given in MZ98a and MZ00 in the case $\delta=0$.

Proof of (i) of Proposition 3. The proof is exactly the same as in the case $\delta=0$ (see page 148 in [MZ98a]). However, one needs the following lower bound, which is a bit tricky to get and which we give for the reader's convenience.

Lemma 4.1 (Sharp lower bound on the blow-up rate). For all $t \in[0, T)$,

$$
\|u(t)\|_{L^{\infty}} \geq \kappa(T-t)^{-\frac{1}{p-1}} .
$$

Remark 4.2. This bound is sharp, since there is equality for the solutions of the ODE $v^{\prime}=(1+i \delta)|v|^{p-1} v$, which are particular solutions of (1.2).

Proof. We introduce $\tilde{\rho}=\sqrt{1+|u|^{2}}$, and we claim that $\tilde{\rho}$ satisfies

$$
\partial_{t} \tilde{\rho} \leq \Delta \tilde{\rho}+\tilde{\rho}^{p} .
$$

Indeed, we can easily prove that $\partial_{t}|u|^{2}=\bar{u} \Delta u+u \Delta \bar{u}+2|u|^{p+1}$. Then we have:

$$
\begin{aligned}
\partial_{t} \tilde{\rho} & =\frac{\partial_{t}|u|^{2}}{2\left(1+|u|^{2}\right)^{1 / 2}}, \\
\Delta \tilde{\rho} & =\frac{\Delta|u|^{2}}{2\left(1+|u|^{2}\right)^{1 / 2}}-\frac{\left.\left.|\nabla| u\right|^{2}\right|^{2}}{4\left(1+|u|^{2}\right)^{3 / 2}} \\
& =\frac{\bar{u} \Delta u+u \Delta \bar{u}+2|\nabla u|^{2}}{2\left(1+|u|^{2}\right)^{1 / 2}}-\frac{|u \cdot \nabla \bar{u}+\bar{u} \cdot \nabla u|^{2}}{4\left(1+|u|^{2}\right)^{3 / 2}} .
\end{aligned}
$$

Using the fact that $|u \cdot \nabla \bar{u}+\bar{u} \cdot \nabla u|^{2} \leq 4|u|^{2}|\nabla u|^{2} \leq 4\left(1+|u|^{2}\right)|\nabla u|^{2}$, we have $\Delta \tilde{\rho} \geq \frac{\bar{u} \Delta u+u \Delta \bar{u}}{2\left(1+|u|^{2}\right)^{1 / 2}}$. Hence

$$
\partial_{t} \tilde{\rho} \leq \Delta \tilde{\rho}+\frac{|u|^{p+1}}{\left(1+|u|^{2}\right)^{1 / 2}} \leq \Delta \tilde{\rho}+\tilde{\rho}^{p},
$$

which gives (4.1).

Now we prove that $\tilde{\rho} \geq \kappa(T-t)^{-\frac{1}{p-1}}$, for all $t \in[0, T)$. For this, we argue by contradiction.

Assume that $\|\tilde{\rho}\|_{L^{\infty}}<\kappa\left(T-t_{0}\right)^{-\frac{1}{p-1}}$, for some $t_{0}<T$. Then, there exists $T_{0}>T$ such that $\left\|\tilde{\rho}\left(t_{0}\right)\right\|_{L^{\infty}} \leq \kappa\left(T_{0}-t_{0}\right)^{-\frac{1}{p-1}}$. Using the maximum principle, the inequality 
remains valid after $T_{0}$ and we have $\lim \sup _{t \rightarrow T}\|\tilde{\rho}(t)\|_{L^{\infty}} \leq \kappa\left(T_{0}-T\right)^{-\frac{1}{p-1}}<\infty$, which is a contradiction. This concludes the proof of Lemma 4.1

Proof of (ii) of Proposition 3. Consider $|\delta| \leq \delta_{0}$ and a solution $u(t)$ of (1.2) that blows up in finite time $T>0$ such that

$$
\forall t \in[0, T),\|u(t)\|_{L^{\infty}} \leq M(\delta)(T-t)^{-\frac{1}{p-1}},
$$

where $\delta_{0}$ and $M(\delta)$ are defined in Theorem 1. Let us now prove the uniform pointwise control of the diffusion term by the nonlinear term, which asserts that the solution $u(t)$ behaves everywhere like the ODE $u^{\prime}=(1+i \delta)|u|^{p-1} u$ (up to a constant).

The plan of the proof is the same as in MZ98a and MZ00. However, the Giga-Kohn property "small local energy implies no blow-up locally" breaks down because we no longer have a gradient structure. The property has to be replaced by a new idea of ours "small $L_{\rho}^{2}$ norm implies no blow-up locally", which is stated in Proposition 3.6

We argue by contradiction and assume that for some $\varepsilon_{0}>0$, there exists $\left(x_{n}, t_{n}\right)_{n \in \mathbb{N}}$, a sequence of elements of $\mathbb{R} \times\left[\frac{T}{2}, T\right)$, such that

$$
\forall n \in \mathbb{N},\left|\Delta u\left(x_{n}, t_{n}\right)\right| \geq \varepsilon_{0}\left|u\left(x_{n}, t_{n}\right)\right|^{p}+n .
$$

From the uniform estimates and the parabolic regularity, since $\|\Delta u\|_{L^{\infty}}$ is bounded on compact sets of $\left[\frac{T}{2}, T\right)$, we have

$$
T-t_{n} \rightarrow 0 \text { as } n \rightarrow \infty .
$$

Part (i) of Proposition 3 implies that $\left|u\left(x_{n}, t_{n}\right)\right|\left(T-t_{n}\right)^{\frac{1}{p-1}}$ is uniformly bounded. Therefore, we can assume that it converges as $n \rightarrow+\infty$. Let us consider two cases:

i) Estimates in the very singular region: $\left|u\left(x_{n}, t_{n}\right)\right|\left(T-t_{n}\right)^{\frac{1}{p-1}} \rightarrow \kappa_{0}>0$. From (4.3), it follows that

$$
\left\|\Delta u\left(t_{n}\right)\right\|_{L^{\infty}} \geq\left|\Delta u\left(x_{n}, t_{n}\right)\right| \geq \varepsilon_{0}\left(\frac{\kappa_{0}}{2}\right)^{p}\left(T-t_{n}\right)^{-\frac{p}{p-1}},
$$

with $t_{n} \rightarrow T$, which contradicts (i) of Proposition 3 ,

ii) Estimates in the singular region: $u\left(x_{n}, t_{n}\right)\left(T-t_{n}\right)^{\frac{1}{p-1}} \rightarrow 0$.

We consider $n$ large enough such that

$$
\left|u\left(x_{n}, t_{n}\right)\right|\left(T-t_{n}\right)^{\frac{1}{p-1}} \leq \frac{\eta_{0}}{3} \text {, where } \eta_{0} \text { is defined in Proposition } 3.6
$$

We take $t_{n}^{0} \rightarrow T$ such that

$$
\left(T-t_{n}^{0}\right)^{-\frac{p}{p-1}}=\sqrt{n}
$$

Using (4.3) and uniform estimates, we obtain

$$
n \leq\left|\Delta u\left(x_{n}, t_{n}\right)\right| \leq C_{0}\left(T-t_{n}\right)^{-\frac{p}{p-1}} ;
$$

hence $t_{n}^{0}<t_{n}$. Now we distinguish two cases:

Case 1. We assume that (up to extracting a subsequence) there exists $t_{n}^{\prime} \in$ $\left(t_{n}^{0}, t_{n}\right)$ such that $\left|u\left(x_{n}, t_{n}^{\prime}\right)\right|\left(T-t_{n}^{\prime}\right)^{\frac{1}{p-1}}=\frac{2}{3} \eta_{0}$. If we consider

$$
v_{n}(\xi, \tau)=\left(T-t_{n}^{\prime}\right)^{\frac{1}{p-1}} u\left(x_{n}+\xi \sqrt{T-t_{n}^{\prime}}, t_{n}^{\prime}+\tau\left(T-t_{n}^{\prime}\right)\right),
$$


then, we have from (i) of Proposition 3 and (4.2),

$$
\left|v_{n}(0,0)\right|=\frac{2}{3} \eta_{0},\left\|\nabla v_{n}(0)\right\|_{L^{\infty}}+\left\|\Delta v_{n}(0)\right\|_{L^{\infty}} \rightarrow 0,
$$

$$
\forall \tau<1,\left\|v_{n}(\tau)\right\|_{L^{\infty}} \leq M(\delta)(1-\tau)^{-\frac{1}{p-1}} \text { and } \partial_{\tau} v_{n}=\Delta v_{n}+(1+i \delta)\left|v_{n}\right|^{p-1} v_{n} .
$$

Using parabolic regularity, we can extract a subsequence (still denoted by $t_{n}$ ) such that, $v_{n}(\xi, \tau) \rightarrow \hat{v}(\xi, \tau)$ in $\mathcal{C}^{2,1}$ for every compact set of $\mathbb{R} \times(-\infty, 1)$, with

$$
\partial_{\tau} \hat{v}=\Delta \hat{v}+(1+i \delta)|\hat{v}|^{p-1} \hat{v},|\hat{v}(0,0)|=2 / 3 \eta_{0} \text { and }\|\hat{v}\|_{L^{\infty}} \leq M(\delta)(1-\tau)^{-\frac{1}{p-1}} .
$$

Using the Liouville theorem (see Theorem 2), we get

$$
\hat{v}(\xi, \tau)=\kappa\left(\left(\frac{3 \kappa}{2 \eta_{0}}\right)^{p-1}-\tau\right)^{-\frac{1+i \delta}{p-1}} e^{i \theta_{0}}, \text { for some } \theta_{0} \in \mathbb{R} .
$$

We claim that it is enough to extend the convergence of $v_{n} \rightarrow \hat{v}$ to all $\tau \in[0,1)$ (and $\xi=0$ ), to conclude the proof. Indeed, if we have this extended convergence, then we write from (4.3) and the definition (4.5) of $v_{n}$,

$$
\left|\Delta v_{n}\left(0, \tau_{n}\right)\right|=\left(T-t_{n}^{\prime}\right)^{\frac{p}{p-1}}\left|\Delta u\left(0, t_{n}\right)\right| \geq \frac{\varepsilon_{0}}{2}\left|u\left(0, t_{n}\right)\right|^{p}\left(T-t_{n}^{\prime}\right)^{\frac{p}{p-1}} \geq \frac{\varepsilon_{0}}{2}\left|v_{n}\left(0, \tau_{n}\right)\right|^{p},
$$

with $\tau_{n}=\frac{t_{n}-t_{n}^{\prime}}{T-t_{n}^{\prime}}$. Letting $n \rightarrow \infty$, we obtain

$$
0 \geq \frac{\varepsilon_{0}}{2} \min _{\tau \in[0,1]}|\hat{v}(\tau)|^{p} \geq \frac{\varepsilon_{0}}{2}\left(\frac{2}{3} \eta_{0}\right)^{p},
$$

which is a contradiction.

Let us then extend the convergence. If we consider the following similarity variables,

$$
y=\frac{\xi-\xi_{0}}{\sqrt{1-\tau}}, s=-\log (1-\tau), w_{n, \xi_{0}}(y, s)=(1-\tau)^{\frac{1}{p-1}} v_{n}(\xi, \tau),
$$

then, we see from (4.6) that for all $\left|\xi_{0}\right| \leq 1,\left\|w_{n, \xi_{0}}(., 0)\right\|_{L_{\rho}^{2}} \leq \eta_{0}$, for $n$ large enough. Using Proposition 3.6, we get for all $|\xi| \leq 1$ and $\tau \in[0,1),\left|v_{n}(\xi, \tau)\right| \leq M_{0}$. Using the parabolic regularity, we can extend the convergence, and then reach the contradiction (4.7). This concludes Case 1.

Case 2. We assume that for some $n_{0} \in \mathbb{N}$, for all $n \geq n_{0}$ and $t \in\left[t_{n}^{0}, t_{n}\right]$, we have

$$
(T-t)^{\frac{1}{p-1}}\left|u\left(x_{n}, t\right)\right|<\frac{2}{3} \eta_{0} .
$$

Then, we take $t_{n}^{\prime}=t_{n}^{0}$ and introduce $v_{n}$ by (4.5). As in Case 1 , we obtain by Proposition 3.6 and parabolic regularity:

$\forall|\xi| \leq 1$ and $\tau \in[0,1),\left|v_{n}(\xi, \tau)\right| \leq M_{0},\left|\Delta v_{n}\left(0, \tau_{n}\right)\right| \leq C_{0} \eta_{0}$, where $\tau_{n}=\frac{t_{n}-t_{n}^{0}}{T-t_{n}^{0}}$.

Therefore, we get from (4.3), (4.5) and (4.4):

$$
n \leq\left|\Delta u_{n}\left(x_{n}, t_{n}\right)\right|=\left(T-t_{n}^{0}\right)^{-\frac{p}{p-1}}\left|\Delta v_{n}\left(0, \tau_{n}\right)\right| \leq C_{0} \eta_{0}\left(T-t_{n}^{0}\right)^{-\frac{p}{p-1}}=C_{0} \eta_{0} \sqrt{n},
$$

which is a contradiction, as $n \rightarrow \infty$. This ends Case 2 and concludes the proof of Proposition 3 , 


\section{Appendix A. Proof of Proposition 3.8}

We prove Proposition 3.8 here. We recall from $(3.12), \ldots,(3.15)$ :

$$
\begin{aligned}
& \tilde{v}_{1 s}=\mathcal{L} \tilde{v}_{1}+\theta^{\prime}(s)\left(\delta \tilde{v}_{1}+\tilde{v}_{2}\right)+\tilde{G}_{1}, \\
& \tilde{v}_{2 s}=(\mathcal{L}-1) \tilde{v}_{2}-\theta^{\prime}(s)\left(\left(1+\delta^{2}\right) \tilde{v}_{1}+\delta \tilde{v}_{2}+\kappa\right)+\tilde{G}_{2},
\end{aligned}
$$

where $\mathcal{L}$ is given in (3.3) and

$$
\begin{aligned}
& \tilde{G}_{1}=\frac{p-\delta^{2}}{2 \kappa} \tilde{v}_{1}^{2}+\frac{1}{2 \kappa} \tilde{v}_{2}^{2}+O\left(|v|^{3}\right), \\
& \tilde{G}_{2}=\left(1+\delta^{2}\right) \frac{\tilde{v}_{1}\left(\delta \tilde{v}_{1}+\tilde{v}_{2}\right)}{\kappa}+O\left(|v|^{3}\right) .
\end{aligned}
$$

A primary idea to deal with system (A.1),..., A.4 is to confirm that it is driven by its linear part $\partial_{s}\left(\tilde{v}_{1}, \tilde{v}_{2}\right)=\left(\mathcal{L} \tilde{v}_{1},(\mathcal{L}-1) \tilde{v}_{2}\right)$ (except for the neutral modes $\tilde{v}_{12}$, where the second-order terms matter, and $\tilde{v}_{20}=0$ by the choice of the modulation parameter; see (2.14)).

To this end, let us decompose $\tilde{v}_{1}$ and $\tilde{v}_{2}$, respectively, with respect to the spectrum of $\mathcal{L}$ (with a positive $(\lambda=1$ or $\lambda=1 / 2$ ), zero and nonnegative part $(\lambda \leq-1 / 2)$ ) and $\mathcal{L}-1$ (with zero eigenvalue and a nonnegative part $(\lambda \leq-1 / 2)$ ). Let us introduce some notation:

$$
\begin{aligned}
& \tilde{v}_{1+}(y, s)=\tilde{v}_{10}(s) h_{0}(y)+\tilde{v}_{11}(s) h_{11}(y), z(s)=\left\|\tilde{v}_{1+}(., s)\right\|_{L_{\rho}^{2}}, \\
& \tilde{v}_{1 \text { null }}(y, s)=\tilde{v}_{12}(s) h_{2}(y), x(s)=\left\|\tilde{v}_{1 \text { null }}(., s)\right\|_{L_{\rho}^{2}}, \\
& \tilde{v}_{1-}(y, s)=\sum_{3}^{+\infty} \tilde{v}_{1 m}(s) h_{m}(y), y_{1}(s)=\left\|\tilde{v}_{1-}(., s)\right\|_{L_{\rho}^{2}},
\end{aligned}
$$

and we denote by

$$
\tilde{v}_{2 \perp}(y, s)=\sum_{1}^{+\infty} \tilde{v}_{2 m}(s) h_{m}(y), \quad y_{2}(s)=\left\|\tilde{v}_{2 \perp}(., s)\right\|_{L_{\rho}^{2}} .
$$

Since we have $\tilde{v}_{20}(s)=0$ from (2.14), it follows that

$$
\tilde{v}_{2 \perp}(y, s)=\tilde{v}_{2}(y, s) \text { and } y_{2}(s)=\left\|\tilde{v}_{2}(., s)\right\|_{L_{\rho}^{2}} .
$$

Finally, we define

$$
\begin{aligned}
& N_{1}(s)=\left\|\theta_{s}\left(\delta \tilde{v}_{1}+\tilde{v}_{2}\right)+\tilde{G}_{1}\right\|_{L_{\rho}^{2}}, \\
& N_{2}(s)=\left\|\theta_{s}\left(\left(1+\delta^{2}\right) \tilde{v}_{1}+\delta \tilde{v}_{2}+\kappa\right)+\tilde{G}_{2}\right\|_{L_{\rho}^{2}} .
\end{aligned}
$$

We proceed in 3 steps:

- In Step 1, we use ODE techniques to show that either $z$ or $x$ dominates as $s \rightarrow-\infty$.

- In Step 2, we consider the case where $z$ dominates and show that it leads to case (i) or (ii) of Proposition 3.8

- In Step 3, we show that (iii) of Proposition 3.8 holds in the case where $x$ dominates.

A.1. Step 1: Either $\left\|\tilde{v}_{1+}(., s)\right\|_{L_{\rho}^{2}}$ or $\left\|\tilde{v}_{1 \text { null }}(., s)\right\|_{L_{\rho}^{2}}$ dominates as $s \rightarrow-\infty$. Projecting (A.1) onto the unstable subspace of $\mathcal{L}$ forming the $L_{\rho}^{2}$-inner product with $\tilde{v}_{1+}$, and using standard inequalities, we get

$$
\dot{z} \geq \frac{1}{2} z-N_{1}
$$


Working similarly with $\tilde{v}_{10}(s), \tilde{v}_{1-}(y, s)$ and $\tilde{v}_{2}(y, s)$ we arrive at the system

$$
\begin{aligned}
& \dot{z} \geq \frac{1}{2} z-N_{1}, \\
& |\dot{x}| \leq N_{1}, \\
& \dot{y_{1}} \leq-\frac{1}{2} y_{1}+N_{1}, \\
& \dot{y}_{2} \leq-\frac{1}{2} y_{2}+N_{2} .
\end{aligned}
$$

Using the fact that $v$ is bounded (see (2.1) and (2.4)), and (2.5), we obtain easily

$$
N_{1}^{2}+N_{2}^{2} \leq C \int|v|^{4} \rho,
$$

for some positive constant $C$. Thus, it follows from that

$$
\begin{aligned}
& \dot{z} \geq \frac{1}{2} z-C N, \\
& |\dot{x}| \leq C N, \\
& \dot{y} \leq-\frac{1}{2} y+C N,
\end{aligned}
$$

where

$$
y \equiv y_{1}+y_{2} \text { and } N^{2} \equiv \int|v|^{4} \rho .
$$

If we knew that for $|s|$ large enough,

$$
N \leq \varepsilon(x+y+z),
$$

which is equivalent to $\int|v|^{4} \rho \leq \varepsilon^{2} \int|v|^{2} \rho$, we could use ODE techniques to conclude the step. The meaning of estimate (A.9) is essentially that the $L_{\rho}^{2}-$ norm of the quadratic term $|v|^{2}$ is small compared to the norm of the linear term $|v|$. However, we do not have this information at this stage. We thus estimate $N$ as follows. Given any $\varepsilon>0$, and any $\alpha>0$ (both will be chosen small in the sequel), there is a time $s_{*}$ such that:

$$
\int|v|^{4} \rho=\int_{|y|>\alpha^{-1}}|v|^{4} \rho+\int_{|y|<\alpha^{-1}}|v|^{4} \rho \leq \alpha^{k} \int|v|^{4}|y|^{k} \rho+\varepsilon^{2} \int|v|^{2} \rho \text { for all } s \leq s_{*} .
$$

Here we use the fact that $v(y, s)$ goes to zero uniformly on the compact set $|y|<$ $\alpha^{-1}$, which follows from (ii) of Lemma 2.4 and parabolic regularity. The exponent $k$ which appears in (A.10) is an arbitrary positive integer (later we will choose it to be large). We set

$$
J^{2} \equiv \int|v|^{4}|y|^{k} \rho,
$$

so that $(\mathrm{A} .10)$ can be rewritten as

$$
\int|v|^{4} \rho \leq \alpha^{k} J^{2}+\varepsilon^{2} \int|v|^{2} \rho \text { for all } s \leq s_{*} .
$$

From the inequalities above, we get that

$$
N \leq \alpha^{k / 2} J+\varepsilon(x+y+z) \text { for all } s \leq s_{*} .
$$

We next estimate $J$. Multiplying (A.1) by $\tilde{v}_{1}|v|^{2}|y|^{k} \rho$, and (3.13) by $\tilde{v}_{2}|v|^{2}|y|^{k} \rho$, integrating over all $\mathbb{R}$, we get after some calculations:

$$
\dot{J} \leq-\theta J+\varepsilon^{\prime}(x+y+z)+c(x+y+z)^{2},
$$

where

$$
\theta=\frac{k}{4}-c-\frac{k \alpha^{2}}{2}(k-1) \text { and } \varepsilon^{\prime}=\frac{1}{2} \varepsilon \alpha^{2-k / 2} k(k+n-2) .
$$


Using the fact that $x, y, z \rightarrow 0$ as $s \rightarrow-\infty$, we end up with

$$
\dot{J} \leq-\theta J+4 \varepsilon^{\prime}(x+y+z),
$$

where $\theta$ is still given by A.12 with a different value of the constant $c$. To end the proof we choose $k$ large enough (certainly $k>4$ ), so that for some $\alpha^{*}(k)>0$, we have for $0<\alpha<\alpha^{*}, \theta \geq \frac{1}{2}$. We obtain from (A.7), (A.11) and (A.13):

where

$$
\begin{aligned}
& \dot{z} \geq\left(\frac{1}{2}-\hat{\varepsilon}\right)-\hat{\varepsilon}(x+\tilde{y}), \\
& |\dot{x}| \leq \hat{\varepsilon}(x+\tilde{y}+z), \\
& \dot{\tilde{y}} \leq-\left(\frac{1}{2}-\hat{\varepsilon}\right) \tilde{y}+\hat{\varepsilon}(x+z),
\end{aligned}
$$

$$
\tilde{y} \equiv y+J, \hat{\epsilon} \equiv C \max \left(\varepsilon+\varepsilon \alpha^{2-k / 2}, \alpha^{k / 2}\right) .
$$

Note that $\hat{\varepsilon}$ can be made arbitrarily small by choosing first $\alpha$ and then $\varepsilon$ sufficiently small. Now, we conclude by using the following lemma.

Lemma A.1. Let $x(s), y(s)$ and $z(s)$ be absolutely continuous, real-valued functions that are nonnegative and satisfy:

i) $(x, y, z)(s) \rightarrow 0$ as $s \rightarrow-\infty$.

ii) For all $\varepsilon>0$, there exists $s_{0} \in \mathbb{R}$ such that for all $s \leq s_{0}$,

$$
\begin{aligned}
& \dot{z} \geq c_{0} z-\varepsilon(x+y), \\
& |\dot{x}| \leq \varepsilon(x+y+z), \\
& \dot{y} \leq-c_{0} y+\varepsilon(x+z) .
\end{aligned}
$$

Then either $x+y=o(z)$ or $y+z=o(x)$, as $s \rightarrow-\infty$.

Proof. Here, we adapt the proof of Lemma A.1 (page 172) from MZ98a. By rescaling in time, we may assume $c_{0}=1$.

Part 1. Let $\varepsilon>0$. We show in this part that either

$$
\exists s_{2}(\varepsilon) \text { such that } \forall s \leq s_{2}, z(s)+y(s) \leq C \varepsilon x(s)
$$

or

$$
\exists s_{2}(\varepsilon) \text { such that } \forall s \leq s_{2}, x(s)+y(s) \leq C \varepsilon z(s) .
$$

We show that for all $s \leq s_{0}(\varepsilon), \beta(s) \leq 0$, where $\beta=y-2 \varepsilon(x+z)$. We argue by contradiction and suppose that there exists $s_{*} \leq s_{0}(\varepsilon)$ such that $\beta\left(s_{*}\right)>0$. Then, if $s \leq s_{*}$ and $\beta(s)>0$, we have from A.14 that $\dot{\beta}=\dot{y}-2 \varepsilon(\dot{x}+\dot{z}) \leq 0$. Therefore, for all $s \leq s_{*}, \beta(s) \geq \beta\left(s_{*}\right)>0$, which contradicts $\beta(s) \rightarrow 0$ as $s \rightarrow-\infty$. Thus, for all $s \leq s_{0}(\varepsilon)$,

$$
y \leq 2 \varepsilon(x+z)
$$

Therefore, (A.14) yields

$$
\begin{aligned}
\dot{z} & \geq \frac{1}{2} z-2 \varepsilon x, \\
|\dot{x}| & \leq 2 \varepsilon(x+z) .
\end{aligned}
$$

Let $\gamma(s)=8 \varepsilon x(s)-z(s)$. Two cases then arise:

- Case 1. There exists $s_{2} \leq s_{0}(\varepsilon)$ such that $\gamma\left(s_{2}\right)>0$. Then we compute $\dot{\gamma}=8 \varepsilon \dot{x}-\dot{z} \leq 16 \varepsilon^{2}(x+z)-\frac{1}{2} z+2 \varepsilon x=\gamma(s)\left(\frac{1}{4}+2 \varepsilon\right)-z(s)\left(\frac{1}{4}-2 \varepsilon-16 \varepsilon^{2}\right)$. Therefore, for all $s \leq s_{2}, \gamma(s) \geq \gamma\left(s_{2}\right) e^{\left(\frac{1}{4}-2 \varepsilon\right)\left(s-s_{2}\right)}>0$, that is, $8 \varepsilon x(s)>$ $z(s)$. Together with (A.17), this yields A.15). 
- Case 2. For all $s \leq s_{0}(\varepsilon), \gamma(s) \leq 0$, that is, $8 \varepsilon x(s) \leq z(s)$. In this case (A.19) yields

$$
\forall s \leq s_{0}(\varepsilon), \dot{z} \geq \frac{1}{4} z \text { and } \dot{x} \leq\left(2 \varepsilon+\frac{1}{4}\right) z ; \text { hence } \dot{x} \leq(1+8 \varepsilon) \dot{z} .
$$

By integration, we get $x(s) \leq(8 \varepsilon+1) z(s)$. We inject this in (A.19) and get from A.20 that $\dot{x} \leq 2 \varepsilon(x+z) \leq 2 \varepsilon z(2+8 \varepsilon) \leq 8 \varepsilon(2+8 \varepsilon) \dot{z}(s)$, which gives $x(s) \leq 8 \varepsilon(2+8 \varepsilon) z(s)$ by integration.

Part 2. It is easy to see that if for some $\varepsilon>0$, A.15) holds, then it holds for all $\varepsilon^{\prime}<\varepsilon$ and the same with A.16. This concludes the proof of Lemma A.1

Applying Lemma A.1 we get either

$$
\left\|\tilde{v}_{12}\right\|_{L_{\rho}^{2}}+\left\|\tilde{v}_{1-}(., s)\right\|_{L_{\rho}^{2}}+\left\|\tilde{v}_{2}(., s)\right\|_{L_{\rho}^{2}}=o\left(\left\|\tilde{v}_{1+}(., s)\right\|_{L_{\rho}^{2}}\right)
$$

or

$$
\left\|\tilde{v}_{1+}(., s)\right\|_{L_{\rho}^{2}}+\left\|\tilde{v}_{1-}(., s)\right\|_{L_{\rho}^{2}}+\left\|\tilde{v}_{2}(., s)\right\|_{L_{\rho}^{2}}=o\left(\left\|\tilde{v}_{12}\right\|_{L_{\rho}^{2}}\right) .
$$

A.2. Step 2: Case where $\left\|\tilde{v}_{1+}(., s)\right\|_{L_{\rho}^{2}}$ dominates. Now, we focus on the case $\left\|\tilde{v}_{1 \text { null }}(., s)\right\|_{L_{\rho}^{2}}+\left\|\tilde{v}_{1-}(., s)\right\|_{L_{\rho}^{2}}+\left\|\tilde{v}_{2}(., s)\right\|_{L_{\rho}^{2}}=o\left(\left\|\tilde{v}_{1+}(., s)\right\|_{L_{\rho}^{2}}\right)$. We will show that it leads to either case (i) or case (ii) of Proposition 3.8. We want to derive from (A.1) the equations satisfied by $\tilde{v}_{10}$ and $\tilde{v}_{11}$. For this, we estimate in the following lemma, $\int \tilde{G}_{1} k_{m}(y) \rho(y) d y$ for $m=0,1$, where

$$
k_{m}(y)=h_{m}(y) /\left\|h_{m}\right\|_{L_{\rho}^{2}}^{2}
$$

and $\tilde{G}_{1}$ is given by (A.3).

Lemma A.2. There exists $\beta_{0}>0$, and an integer $k^{\prime}>4$ such that for all $\beta \in$ $\left(0, \beta_{0}\right), \exists s_{0} \in \mathbb{R}$ such that $\forall s \leq s_{0},\left.\int v^{2}|y|\right|^{k^{\prime}} \rho \leq c_{0}\left(k^{\prime}\right) \beta^{4-k^{\prime}} z(s)^{2}$.

Proof. This lemma is analogous to Lemma A.3, p. 175 from [MZ98a, which handles the real case with $\delta=0$. One can adapt with no difficulty the proof of the present context.

Proceeding as in Appendix A from [MZ98a and doing the projection of equation (3.12), respectively on $k_{0}(y)$ and $k_{1}(y)$, we obtain

$$
\tilde{v}_{10}^{\prime}(s)=\tilde{v}_{10}(s)+\frac{p-\delta^{2}}{2 \kappa}(1+\alpha(s)) z^{2}(s)
$$

and

$$
\tilde{v}_{11}^{\prime}(s)=\frac{1}{2} \tilde{v}_{11}(s)+\eta(s) z(s)^{2},
$$

where $z(s)=\left\|\tilde{v}_{1+}(., s)\right\|_{L_{\rho}^{2}}, \alpha(s) \rightarrow 0$ as $s \rightarrow+\infty$ and $\eta$ is bounded. Then, from standard ODE techniques, we get

$$
\forall \varepsilon>0, \tilde{v}_{10}(s)=O\left(e^{(1-\varepsilon) s}\right) \text { and } \tilde{v}_{11}=C_{1} e^{\frac{s}{2}}+O\left(e^{(1-\varepsilon) s}\right) .
$$

Since $z(s)^{2}=\left\|\tilde{v}_{1+}(., s)\right\|_{L_{\rho}^{2}}^{2}=\tilde{v}_{10}^{2}+2 \tilde{v}_{11}^{2}$, we write (A.21) as

$$
\tilde{v}_{10}^{\prime}(s)=\tilde{v}_{10}(s)+\frac{p-\delta^{2}}{\kappa}\left|C_{1}\right|^{2} s e^{s}(1+\alpha(s))+\gamma(s),
$$

where $\gamma(s)=O\left(e^{2(1-\varepsilon) s}\right)$ and $\alpha(s) \rightarrow 0$ as $s \rightarrow-\infty$, which gives by integration

$$
\tilde{v}_{10}(s)=\frac{p-\delta^{2}}{\kappa}\left|C_{1}\right|^{2} s e^{s}(1+o(s))+C_{0} e^{s}+O\left(e^{2(1-\varepsilon)}\right), \text { as } s \rightarrow-\infty .
$$


Two cases then arise:

- If $C_{1} \neq 0$, then $\tilde{v}_{11} \equiv C_{1} e^{\frac{s}{2}} \gg \tilde{v}_{10}=O\left(s e^{s}\right)$, from A.24. Note first that applying Lemma 3.7 to $\left|v_{1}\right|+\left|v_{2}\right|$ (this is possible from equations (A.1) and (A.2) and the boundedness of $v$ ), we have for all $|s|$ large enough (and $s<0)$,

$$
N^{2}=\int|v(y, s)|^{4} \rho(y) d y \leq C *\left\|v\left(., s-s^{*}\right)\right\|_{L_{\rho}^{2}}^{2},
$$

for some positive $s^{*}$ and $C^{*}$.

Recalling system A.7 and using A.25, we obtain $\dot{y} \leq-\frac{1}{2} y+$ $c\left\|v\left(., s-s^{*}\right)\right\|_{L_{\rho}^{2}}^{2} \leq-\frac{1}{2} y+c e^{s}$. Then, we obtain $y=O\left(e^{s}\right)$; similarly, we obtain $x=\left\|\tilde{v}_{1 \text { null }}(., s)\right\|_{L_{\rho}^{2}}=O\left(e^{s}\right)$. We conclude that $\| v(., s)-(1+$ $i \delta) C_{1} e^{s / 2} y \|_{L_{\rho}^{2}}=O\left(e^{s(1-\varepsilon)}\right)$ as $s \rightarrow-\infty$, for some $\varepsilon>0$. Using (2.5), we get $\left|\theta_{s}\right| \leq C e^{s}$. This is case (ii) of Proposition 3.8.

- If $C_{1}=0$, we obtain case (i) of Proposition 3.8. Indeed, let us first improve the estimate of $v$. In fact, from (A.24 wave $\tilde{v}_{10}=C_{0} e^{s}+O\left(e^{3 / 2 s}\right)$ and from (A.22) we have $\tilde{v}_{11}=O\left(e^{3 / 2 s}\right)$.

We note $y=\left\|\tilde{v}_{1-}(., s)\right\|_{L_{\rho}^{2}}+\left\|\tilde{v}_{2}(., s)\right\|_{L_{\rho}^{2}}$ and $x=\left\|\tilde{v}_{1 n u l l}(., s)\right\|_{L_{\rho}^{2}}$. Recalling system (A.7) and using (A.25), we obtain

$$
\dot{y} \leq-\frac{1}{2} y+c\left\|v\left(., s-s^{*}\right)\right\|_{L_{\rho}^{2}}^{2} \leq-\frac{1}{2} y+c e^{2 s} .
$$

Then, we have that $y=\left\|\tilde{v}_{1-}(., s)\right\|_{L_{\rho}^{2}}+\left\|\tilde{v}_{2}(., s)\right\|_{L_{\rho}^{2}}=O\left(e^{3 / 2 s}\right)$. Similarly, we obtain that

$$
x=\left\|\tilde{v}_{1 n u l l}(., s)\right\|_{L_{\rho}^{2}}=O\left(e^{3 / 2 s}\right)
$$

and we conclude

$$
\begin{aligned}
& \left\|v(., s)-(1+i \delta) \tilde{v}_{10}(s)\right\|_{L_{\rho}^{2}} \\
& =\left\|(1+i \delta)\left(\tilde{v}_{11}(s)+\tilde{v}_{1, \text { null }}(., s)+\tilde{v}_{1-}(., s)\right)+i \tilde{v}_{2}(., s)\right\|_{L_{\rho}^{2}} \\
& =O\left(e^{3 / 2 s}\right) .
\end{aligned}
$$

Using (2.5), we get $\left|\theta^{\prime}(s)\right|_{L_{\rho}^{2}} \leq C e^{2 s}$. This is case (i) of Proposition 3.8 .

A.3. Step 3: Case where $\left\|\tilde{v}_{1 n u l l}(., s)\right\|_{L_{\rho}^{2}}$ dominates. In the following we prove that (iii) of Proposition 3.8 holds. First, we prove the following lemma:

Lemma A.3. Assume that

$$
\left\|\tilde{v}_{1+}(., s)\right\|_{L_{\rho}^{2}}+\left\|\tilde{v}_{1-}(., s)\right\|_{L_{\rho}^{2}}+\left\|\tilde{v}_{2}(., s)\right\|_{L_{\rho}^{2}}=o\left(\left\|\tilde{v}_{1 n u l l}(., s)\right\|_{L_{\rho}^{2}}\right)
$$

holds. Then

$$
v(y, s)=-(1+i \delta) \frac{\kappa}{4\left(p-\delta^{2}\right) s}\left(y^{2}-2\right)+o\left(\frac{1}{s}\right),
$$

in $L_{\rho}^{2}$ as $s \rightarrow-\infty$. 
Proof. Since $\tilde{v}_{1 \text { null }}=\tilde{v}_{12}(s) h_{2}(y)$, we note that $\tilde{v}_{12}=\int \tilde{v}_{1} k_{2} \rho$. Projecting equation (A.1) onto $h_{2}(y)$ we get

$$
\begin{aligned}
\frac{d}{d s}\left(\tilde{v}_{12}\right)= & \frac{p-\delta^{2}}{2 \kappa} \int \tilde{v}_{1}^{2} k_{2}(y) \rho(y) \\
& +\theta^{\prime}(s) \int\left(\delta \tilde{v}_{1}+\tilde{v}_{2}\right) k_{2}(y) \rho(y)+\int \frac{1}{2 \kappa} \tilde{v}_{2}^{2} k_{2}(y) \rho(y)+O\left(\int|v|^{3} k_{2}(y) \rho(y)\right), \\
= & \frac{p-\delta^{2}}{2 \kappa} \int \tilde{v}_{1 \text { null }}^{2} k_{2}(y) \rho(y)-\frac{p-\delta^{2}}{2 \kappa} \int\left(\tilde{v}_{1 \text { null }}^{2}-\tilde{v}_{1}^{2}\right) k_{2}(y) \rho(y) \\
& +\theta^{\prime}(s) \int\left(\delta \tilde{v}_{1}+\tilde{v}_{2}\right) k_{2}(y) \rho(y)+\int \frac{1}{2 \kappa} \tilde{v}_{2}^{2} k_{2}(y) \rho(y)+O\left(\int|v|^{3} k_{2}(y) \rho(y)\right), \\
\equiv & \frac{\left(p-\delta^{2}\right)}{2 \kappa} 8 \tilde{v}_{12}^{2}+\frac{p-\delta^{2}}{2 \kappa} \mathcal{E}_{1}+\mathcal{E}_{2}+\mathcal{E}_{3}+\mathcal{E}_{4},
\end{aligned}
$$

where we use the fact that $\int \tilde{v}_{\text {null }} k_{2} \rho=\tilde{v}_{12}^{2} \int h_{2}^{2} k_{2} \rho=8 \tilde{v}_{12}^{2}$. We next estimate $\mathcal{E}_{1}$, $\mathcal{E}_{2}, \mathcal{E}_{3}$ and $\mathcal{E}_{4}$. For this, we need the following lemma:

Lemma A.4. There exist $\alpha_{0}>0$ and an integer $k^{\prime}>4$ such that for all $\alpha \in\left(0, \alpha_{0}\right)$, there exists $s_{0} \in \mathbb{R}$ such that for all $s \leq s_{0}$,

$$
\int|v|^{2}|y|^{k^{\prime}} \rho d y \leq c_{0}\left(k^{\prime}\right) \alpha^{4-k^{\prime}} \int \tilde{v}_{1 n u l l}^{2} \rho d y \text {. }
$$

Proof. See the proof of Lemma C.1 in [MZ98a] (page 187).

Recalling that $\tilde{v}_{1}=\tilde{v}_{1-}+\tilde{v}_{1+}+\tilde{v}_{1 n u l l}$, we write on the one hand:

$$
\begin{aligned}
\left|\mathcal{E}_{1}\right| & \leq \int\left|\tilde{v}_{1+}+\tilde{v}_{1-}\right| \times\left|\tilde{v}_{1}+\tilde{v}_{1 \text { null }}\right|\left|k_{2}(y)\right| \rho, \\
& \leq c\left(\int\left|\tilde{v}_{1+}+\tilde{v}_{1-}\right|^{2} \rho\right)^{1 / 2}\left\{\left(\int \tilde{v}_{1}^{2} k_{2}^{2}(y) \rho\right)^{1 / 2}+\left(\int \tilde{v}_{1 \text { null }}^{2} k_{2}^{2}(y) \rho\right)^{1 / 2}\right\} .
\end{aligned}
$$

We have from (A.26) that $\left(\int\left|\tilde{v}_{1+}+\tilde{v}_{1-}\right|^{2} \rho\right)^{1 / 2}=o\left(\tilde{v}_{12}\right)$ and

$$
\left(\int \tilde{v}_{1}^{2} k_{2}^{2}(y) \rho\right)^{1 / 2}+\left(\int \tilde{v}_{1 \text { null }}^{2} k_{2}^{2}(y) \rho\right)^{1 / 2} \leq\left(\int|v|^{2} k_{2}^{2} \rho\right)^{1 / 2}+c\left|\tilde{v}_{12}\right| \equiv I_{1}+I_{2} .
$$

On the other hand, we have

$$
\begin{aligned}
\mathcal{E}_{3}=\int \frac{1}{2 \kappa} \tilde{v}_{2}^{2} k_{2}(y) \rho(y) & \leq c\left(\int \tilde{v}_{2}^{2} \rho\right)^{1 / 2}\left(\int \tilde{v}_{2}^{2} k_{2}^{2} \rho\right)^{1 / 2} \\
& \leq o\left(\tilde{v}_{12}\right) \underbrace{\left(\int|v|^{2} k_{2}^{2} \rho\right)^{1 / 2}}_{I_{1}} .
\end{aligned}
$$

To treat $I_{1}$, we have from A.4

$$
\int \tilde{v}_{1}^{2} k_{2}^{2} \rho \leq c \int|v|^{2} \rho+c \int|v|^{2}|y|^{k^{\prime}} \rho \leq c\left(\int|v|^{2} \rho\right) \leq c \tilde{v}_{12}^{2} .
$$

We conclude that $\mathcal{E}_{1}=o\left(\tilde{v}_{12}^{2}\right)$ and $\mathcal{E}_{3}=o\left(\tilde{v}_{12}^{2}\right)$. We can see easily that $\mathcal{E}_{2}=o\left(\tilde{v}_{12}^{2}\right)$, because of Lemma 2.4 . 
It remains to estimate $\mathcal{E}_{4}$. We consider $\alpha \in\left(0, \alpha_{0}\right)$ and we proceed as in Appendix C from MZ98a] (page 189). We write for $m=0$ or $m=2$ :

$$
\begin{aligned}
\int|v|^{3}|y|^{m} \rho d y & \leq \int_{|y| \leq \alpha^{-1}}|v|^{3}|y|^{m} \rho d y+\int_{|y| \geq \alpha^{-1}}|v|^{3}|y|^{m} \rho d y \\
& \leq \varepsilon \alpha^{-m} \int_{|y| \leq \alpha^{-1}}|v|^{2} \rho d y+C M \alpha^{k^{\prime}-m} \int_{|y| \geq \alpha^{-1}}|v|^{2}|y|^{k^{\prime}} \rho d y \\
& \leq C\left(\varepsilon \alpha^{-m}+M c_{0}\left(k^{\prime}\right) \alpha^{4-m}\right) \int \tilde{v}_{\text {null }}^{2} \rho d y
\end{aligned}
$$

where we used the fact that $|v| \rightarrow 0$ as $s \rightarrow-\infty$ in $L^{\infty}\left(B\left(0, \alpha^{-1}\right)\right),|v(y, s)| \leq M$, Lemma A.4 and $\int|v|^{2} \rho d y \leq \int \tilde{v}_{\text {null }}^{2} \rho d y$. We can then choose $\varepsilon$ and $\alpha$ such that for $s \leq s_{0}, \int|v|^{3}|y|^{m} \rho \leq \varepsilon \int \tilde{v}_{\text {null }}^{2} \rho$ and we obtain $\mathcal{E}_{4}=o\left(\tilde{v}_{12}^{2}\right)$.

So finally, we have

$$
\frac{d}{d s}\left(\tilde{v}_{12}\right)=\frac{\left(p-\delta^{2}\right)}{\kappa} 4 \tilde{v}_{12}^{2}+o\left(\tilde{v}_{12}^{2}\right)
$$

Solving the above, we obtain

$$
\tilde{v}_{1 n u l l}=-\frac{\kappa}{4\left(p-\delta^{2}\right) s}(1+o(1))\left(y^{2}-2\right) .
$$

This concludes the proof of Lemma A.3.

In order to finish the proof of (iii) of Proposition 3.8, we need to refine the estimates of Lemma A.3 to catch the $O\left(\frac{|s|}{s^{2}}\right)$.

Recalling system (A.7) and using (A.25), we obtain

$$
y^{\prime} \leq-\frac{1}{2} y+c\left\|v\left(., s-s^{*}\right)\right\|_{L_{\rho}^{2}}^{2} \leq-\frac{1}{2} y+c \frac{1}{s^{2}} .
$$

Then, integrating $\left(y e^{s / 2}\right)^{\prime} \leq C \frac{e^{s / 2}}{s^{2}}$ between $-\infty$ and $s$, we get $y \leq \frac{C}{s^{2}}$. Doing the same for $z=\left\|\tilde{v}_{1+}(., s)\right\|_{L_{\rho}^{2}}$, we obtain $\left(z e^{-s / 2}\right)^{\prime} \geq C \frac{e^{s / 2}}{s^{2}}$. Integrating between $s$ and $s_{0} \geq s$, we have $z \leq \frac{C}{s^{2}}$.

Proceeding as in the proof of Proposition A.3, we write:

$$
\begin{aligned}
& \frac{d}{d s}\left(\tilde{v}_{12}\right)=\frac{p-\delta^{2}}{2 \kappa} \int \tilde{v}_{1 \text { null }}^{2} k_{2}(y) \rho(y)-\frac{p-\delta^{2}}{2 \kappa} \int\left(\tilde{v}_{1 \text { null }}^{2}-\tilde{v}_{1}^{2}\right) k_{2}(y) \rho(y) \\
& +\theta^{\prime}(s) \int\left(\delta \tilde{v}_{1}+\tilde{v}_{2}\right) k_{2}(y) \rho(y)+\int \frac{1}{2 \kappa} \tilde{v}_{2}^{2} k_{2}(y) \rho(y)+O\left(\int|v|^{3} k_{2}(y) \rho(y)\right) \\
& \equiv \frac{4\left(p-\delta^{2}\right)}{\kappa} \tilde{v}_{12}^{2}+\frac{p-\delta^{2}}{2 \kappa} \mathcal{E}_{1}+\mathcal{E}_{2}+\mathcal{E}_{3}+\mathcal{E}_{4} .
\end{aligned}
$$


Then, we have

$$
\begin{aligned}
\left|\mathcal{E}_{1}\right| & \leq \int\left|\tilde{v}_{1+}+\tilde{v}_{1-}+\tilde{v}_{2}\right| \times\left|v+\tilde{v}_{1 \text { null }}\right|\left|k_{2}(y)\right| \rho \\
& \leq\left(\int\left|\tilde{v}_{1+}+\tilde{v}_{1-}+\tilde{v}_{2}\right|^{2} \rho\right)^{1 / 2}\left\{\left(\int v^{2} k_{2}^{2}(y) \rho\right)^{1 / 2}+\left(\int \tilde{v}_{1 \text { null }}^{2} k_{2}^{2}(y) \rho\right)^{1 / 2}\right\} \\
& \leq \varepsilon\left(\int \tilde{v}_{1 \text { null }}^{2} \rho\right)^{1 / 2}\left\{c\left(\int v^{4} \rho\right)^{1 / 4}+c\left(\int \tilde{v}_{1 \text { null }}^{2} \rho\right)^{1 / 2}\right\} .
\end{aligned}
$$

Using the fact that $\left\|\tilde{v}_{1 n u l l}(., s)\right\|_{L_{\rho}^{2}} \sim \frac{C}{s}$ and (A.25), we have

$$
\int v^{4} \rho \leq c\left(\int v^{2}\left(., s-s^{*}\right) \rho\right)^{2} \leq \frac{c}{\left(s-s^{*}\right)^{2}} \leq \frac{c}{s^{2}} .
$$

Thus, $\mathcal{E}_{1} \leq \frac{C}{s^{3}}$. Similarly, we obtain $\mathcal{E}_{2} \leq \frac{C}{|s|^{3}}, \mathcal{E}_{3} \leq y^{2} \leq \frac{C}{s^{4}}$ and $\mathcal{E}_{4} \leq \frac{C}{|s|^{3}}$. Then, we have from (A.27):

$$
\frac{d}{d s}\left(\tilde{v}_{12}\right)=\frac{4\left(p-\delta^{2}\right)}{\kappa} \tilde{v}_{12}^{2}+O\left(\frac{1}{s^{3}}\right)=\frac{4\left(p-\delta^{2}\right)}{\kappa} \tilde{v}_{12}^{2}\left(1+O\left(\frac{1}{s}\right)\right) .
$$

By integrating, we conclude that:

$$
\tilde{v}_{12}=-\frac{\kappa}{4\left(p-\delta^{2}\right) s}+O\left(\frac{\log |s|}{s^{2}}\right) .
$$

Finally, we get $\left\|v(., s)-(1+i \delta) \frac{\kappa}{4\left(p-\delta^{2}\right) s}\left(y^{2}-2\right)\right\|_{L_{\rho}^{2}}=O\left(\frac{\log |s|}{s^{2}}\right)$ as $s \rightarrow-\infty$. It remains to prove the estimate for $\theta^{\prime}(s)$ to conclude the proof.

Integrating equation (A.2) with respect to $\rho d y$, we obtain:

$$
\theta^{\prime}(s) \int\left(\left(1+\delta^{2}\right) \tilde{v}_{1}+\delta \tilde{v}_{2}+\kappa\right) \rho=\int \tilde{G}_{2} \rho
$$

On the one hand, we have $\left(\left(1+\delta^{2}\right) \tilde{v}_{1}+\delta \tilde{v}_{2}+\kappa\right)=\kappa+O\left(\frac{1}{s}\right)$. On the other hand, using (A.4), we get

$$
\int \tilde{G}_{2} \rho=\frac{\left(1+\delta^{2}\right) \delta}{\kappa} \int \tilde{v}_{1}^{2} \rho+\frac{\left(1+\delta^{2}\right)}{\kappa} \int \tilde{v}_{1} \tilde{v}_{2} \rho
$$

where we have from (iii) of Proposition 3.8 $\int \tilde{v}_{1} \tilde{v}_{2} \rho=O\left(\frac{\log |s|}{s^{3}}\right)$,

$$
\begin{gathered}
\int \tilde{v}_{1}^{2} \rho=\int \tilde{v}_{12}^{2} h_{2}^{2} \rho+\int\left(\tilde{v}_{1}^{2}-\tilde{v}_{12}^{2} h_{2}^{2}\right) \rho \\
=8 \tilde{v}_{12}^{2}+\int\left(\left(\tilde{v}_{1}-\tilde{v}_{12} h_{2}\right)\right)\left(\tilde{v}_{1}+\tilde{v}_{12}\right) \rho, \\
\tilde{v}_{12}=\frac{\kappa}{4\left(p-\delta^{2}\right) s}+O\left(\frac{\log |s|}{s^{2}}\right), \int\left(\left(\tilde{v}_{1}-\tilde{v}_{12} h_{2}\right)\right)\left(\tilde{v}_{1}+\tilde{v}_{12}\right) \rho \leq C \frac{\log |s|}{s^{2}} \times \frac{1}{s}=C \frac{\log |s|}{s^{3}}, \\
\theta^{\prime}(s)=\frac{\left(1+\delta^{2}\right) \delta}{\kappa}\left(\frac{\kappa}{2\left(p-\delta^{2}\right)}\right)^{2} \frac{1}{s^{2}}+O\left(\frac{\log |s|}{s^{3}}\right) .
\end{gathered}
$$

Consequently, we obtain the desired estimate for $\theta_{s}$. This concludes the proof of Proposition 3.8 . 


\section{Appendix B. Equations of $Z$ in Steps 4 and 5}

Equation of $Z$ in Step 4: In this part we establish the equation satisfied by $Z$ in Step 4 of the proof of Theorem 1 . We denote by $\phi: \mathbb{C} \rightarrow \mathbb{C}$ the function defined by $\phi(x)=|x|^{p-1} x$. If we introduce $\nu=(w-F)$, where $F$ is defined by (3.30), then we see from (1.10) that $\nu$ satisfies the following equation for all $(y, s) \in \mathbb{R} \times \mathbb{R}$, such that for $|y|<4 \varepsilon_{0} \sqrt{-s}$,

$$
\partial_{s} \nu=(\mathcal{L}-1) \nu+l(\nu)+B(\nu)+R(y, s),
$$

where $\mathcal{L}$ is defined in (3.3), $\nu=\nu_{1}+i \nu_{2}$,

$$
\begin{aligned}
l(\nu) & =(1+i \delta)\left[-\frac{\nu}{p-1}+(p-1)|F|^{p-3} F\left(F_{1} \nu_{1}+F_{2} \nu_{2}\right)+|F|^{p-1} \nu\right], \\
B(\nu) & =(1+i \delta)\left[\phi(F+\nu)-\phi(F)-(p-1)|F|^{p-3} F\left(F_{1} \nu_{1}+F_{2} \nu_{2}\right)-|F|^{p-1} \nu\right], \\
R(y, s) & =-\partial_{s} F+\Delta F-\frac{1}{2} y \cdot \nabla F-(1+i \delta) \frac{F}{p-1}+(1+i \delta)|F|^{p-1} F .
\end{aligned}
$$

Using Taylor's formula and the fact that $w$ and $F$ are bounded for $|y| \leq 4 \varepsilon_{0} \sqrt{-s}$, we readily obtain for all $s \leq s_{0}$ and $|y|<4 \varepsilon_{0} \sqrt{-s}$ that

$$
\begin{aligned}
& |B(\nu)| \leq C|\nu|^{2}, \\
& |R(y, s)| \leq C\left(\frac{|y|^{2}+1}{s^{2}}+\chi_{\varepsilon_{0}}\right),
\end{aligned}
$$

with $\chi_{\varepsilon_{0}}$ defined in (3.32). If we write $\nu=(1+i \delta) \tilde{\nu}_{1}+i \tilde{\nu}_{2}, B=(1+i \delta) \tilde{B}_{1}+i \tilde{B}_{2}$ and $R=(1+i \delta) \tilde{R}_{1}+i \tilde{R}_{2}$, then we have

$$
\begin{aligned}
\partial_{s} \tilde{\nu}_{1} & =\mathcal{L} \tilde{\nu}_{1}+l_{1,1} \tilde{\nu}_{1}+l_{1,2} \tilde{\nu}_{2}+\tilde{B}_{1}+\tilde{R}_{1}, \\
\partial_{s} \tilde{\nu}_{2} & =(\mathcal{L}-1) \tilde{\nu}_{2}+l_{2,2} \tilde{\nu}_{2}+l_{2,1} \tilde{\nu}_{1}+\tilde{B}_{2}+\tilde{R}_{2},
\end{aligned}
$$

where

$$
\left\{\begin{array}{l}
l_{1,1}(y, s)=\left(1-\delta^{2}\right)\left(|F|^{p-1}-\frac{1}{p-1}\right)+(p-1)|F|^{p-3}\left(F_{1}{ }^{2}-\delta^{2} F_{2}{ }^{2}\right)-1 \\
l_{1,2}(y, s)=-\delta\left(|F|^{p-1}-\frac{1}{p-1}\right)+(p-1)|F|^{p-3}\left(F_{1}-\delta F_{2}\right) F_{2} \\
l_{2,1}(y, s)=\left(1+\delta^{2}\right)\left(|F|^{p-1}-\frac{1}{p-1}\right)+(p-1)|F|^{p-3}\left(F_{1}+\delta F_{2}\right) F_{2} \\
l_{2,2}(y, s)=\left(1+\delta^{2}\right)\left(|F|^{p-1}-\frac{1}{p-1}\right)+(p-1)|F|^{p-3} F_{2}{ }^{2}
\end{array}\right.
$$

Proceeding as in the proof of Lemma B.1 from [Zaa98] (page 615), we obtain for all $|y| \leq 4 \varepsilon_{0} \sqrt{-s}$

$$
\left|l_{i, j}(y, s)\right| \leq C \min \left[\frac{\left(1+\left|y^{2}\right|\right)}{|s|}, 1\right], \text { for any } i, j \in\{1,2\} .
$$

Therefore, we write for $|s|$ large enough and $|y| \leq 4 \varepsilon_{0} \sqrt{-s}$ :

$$
\left|l_{i, j}(y, s)\right| \leq C\left\{\frac{\left(1+\varepsilon_{0}^{2}|s|\right)}{|s|}+\chi_{\varepsilon_{0}}\right\} \leq C\left\{2 \varepsilon_{0}^{2}+\chi_{\varepsilon_{0}}\right\}
$$


Now, we multiply (B.2) and (B.3) respectively by $\operatorname{sgn}\left(\tilde{\nu}_{1}\right)$ and $\operatorname{sgn}\left(\tilde{\nu}_{2}\right)$. Using Kato's inequality, we obtain for $z=\left|\tilde{\nu}_{1}\right|+\left|\tilde{\nu}_{2}\right|,|s|$ large enough and $|y| \leq 4 \varepsilon_{0} \sqrt{-s}$ :

$$
\partial_{s} z-\Delta z+\frac{1}{2} y \cdot \nabla z-(1+\sigma) z \leq C\left(z^{2}+\frac{\left(y^{2}+1\right)}{s^{2}}+\chi_{\varepsilon_{0}}\right),
$$

where we fix $\varepsilon_{0}$ small enough so that $\sigma=C \varepsilon_{0}^{2}=\frac{1}{100}$.

Now, we consider the cutoff function $\gamma$ (3.33), we define $Z=z \gamma$ and we obtain for $|s|$ large enough:

$$
\begin{aligned}
\partial_{s} Z-\Delta Z+\frac{1}{2} y \cdot \nabla Z-(1+\sigma) Z & \leq C\left(Z^{2}+\frac{\left(y^{2}+1\right)}{s^{2}}+\chi_{\varepsilon_{0}}\right) \\
& +z\left(\partial_{s} \gamma-\Delta \gamma+\frac{y}{2} \cdot \nabla \gamma\right)-2 \nabla \gamma \cdot \nabla z
\end{aligned}
$$

(here, we used the fact that $\left.\gamma z^{2}=Z^{2}+\left(\gamma-\gamma^{2}\right) z^{2} \leq Z^{2}+C \chi_{\varepsilon_{0}}\right)$. The last terms in this equation are the cutoff terms. Using the fact that $z\left(\partial_{s} \gamma-\Delta \gamma+\frac{y}{2} \cdot \nabla \gamma\right)-$ $2 \nabla \gamma \nabla z \leq C \chi_{\varepsilon_{0}}-2 \operatorname{div}(z \nabla \gamma)$, we obtain for $|s|$ large enough:

$\partial_{s} Z-\Delta Z+\frac{1}{2} y \cdot \nabla Z-(1+\sigma) Z \leq C\left(Z^{2}+\frac{\left(y^{2}+1\right)}{s^{2}}+\chi_{\varepsilon_{0}}\right)-2 \operatorname{div}\left(\left(\left|\tilde{\nu}_{1}\right|+\left|\tilde{\nu}_{2}\right|\right) \nabla \gamma\right)$,

which is the desired equation in Lemma 3.11,

Equation of $Z$ in Step 4: In the following, we determine the equation satisfied by $Z$ in Step 4 . We let $\nu=w-f$. We can see from (3.46) that $\nu$ satisfies the following equation for all $(y, s) \in \mathbb{R} \times \mathbb{R}$, such that for $|y|<4 \varepsilon_{0} e^{-s / 2}$,

$$
\partial_{s} \nu=\Delta \nu-\frac{1}{2} y \cdot \nabla \nu+l(\nu)+B(\nu)+R(y, s),
$$

where

$$
\begin{gathered}
l(\nu)=-(1+i \delta) \frac{\nu}{p-1}+(1+i \delta)\left\{(p-1)|f|^{p-3} f\left(f_{1} \nu_{1}+f_{2} \nu_{2}\right)+|f|^{p-1} \nu\right\} \\
\begin{aligned}
B(\nu)=(1+i \delta)\left\{|f+\nu|^{p-1}(f+\nu)-|f|^{p-1} f\right. \\
\left.-(p-1)|f|^{p-3} f\left(f_{1} \nu_{1}+f_{2} \nu_{2}\right)-|f|^{p-1} \nu\right\} \\
R(y, s)=e^{s} \Delta G\left(y e^{s / 2}\right) .
\end{aligned}
\end{gathered}
$$

Using a Taylor formula, we prove that for $|s|$ large and $|y| \leq 4 \varepsilon_{0} e^{-s / 2}$,

$$
|B(\nu)| \leq C|\nu|^{2}, \quad|R(y, s)| \leq C e^{s}+\chi_{\varepsilon_{0}}(y, s),
$$

with $\chi_{\varepsilon_{0}}$ defined by (3.49). If we write $\nu=(1+i \delta) \tilde{\nu}_{1}+i \tilde{\nu}_{2}, B=(1+i \delta) \tilde{B}_{1}+i \tilde{B}_{2}$ and $R=(1+i \delta) \tilde{R}_{1}+i \tilde{R}_{2}$, then we have

$$
\begin{aligned}
& \partial_{s} \tilde{\nu}_{1}=\mathcal{L} \tilde{\nu}_{1}+l_{1,1} \tilde{\nu}_{1}+l_{1,2} \tilde{\nu}_{2}+\tilde{B}_{1}+\tilde{R}_{1}, \\
& \partial_{s} \tilde{\bar{\nu}}_{2}=(\mathcal{L}-1) \tilde{\bar{\nu}}_{2}+l_{2,2} \tilde{\nu}_{2}+l_{2,1} \tilde{\nu}_{1}+\tilde{B}_{2}+\tilde{R}_{2},
\end{aligned}
$$

where

$$
\left\{\begin{array}{l}
l_{1,1}(y, s)=\left(1-\delta^{2}\right)\left(|f|^{p-1}-\frac{1}{p-1}\right)+(p-1)|f|^{p-3}\left(f_{1}{ }^{2}-\delta^{2} f_{2}{ }^{2}\right)-1, \\
l_{1,2}(y, s)=-\delta\left(|f|^{p-1}-\frac{1}{p-1}\right)+(p-1)|f|^{p-3}\left(f_{1}-\delta f_{2}\right) f_{2}, \\
l_{2,1}(y, s)=\left(1+\delta^{2}\right)\left(|f|^{p-1}-\frac{1}{p-1}\right)+(p-1)|f|^{p-3}\left(f_{1}+\delta f_{2}\right) f_{2}, \\
l_{2,2}(y, s)=\left(1+\delta^{2}\right)\left(|f|^{p-1}-\frac{1}{p-1}\right)+(p-1)|f|^{p-3} f_{2}{ }^{2} .
\end{array}\right.
$$

Proceeding as in the proof of Lemma B.1 from [Zaa98] (page 615), we obtain for $|y| e^{s / 2} \leq 4 \varepsilon_{0}$ and $s$ large,

$$
\left|l_{i, j}(y, s)\right| \leq C \min \left[|y| e^{s / 2}, 1\right], \text { for any } i, j \in\{1,2\} .
$$


If we consider $\chi_{\varepsilon_{0}}$ defined in (3.49), then we write for $|s|$ large and $|y| \leq 4 \varepsilon_{0} e^{-s / 2}$ :

$$
\left|l_{i, j}\right| \leq C\left\{|y| e^{s / 2}+\chi_{\varepsilon_{0}}\right\} \leq C\left\{\varepsilon_{0}+\chi_{\varepsilon_{0}}\right\} .
$$

Now, we multiply (B.4) and (B.5) respectively by $\operatorname{sgn}\left(\tilde{\nu}_{1}\right)$ and $\operatorname{sgn}\left(\tilde{\nu}_{2}\right)$. Using Kato's inequality, we obtain for $z=\left|\tilde{\nu}_{1}\right|+\left|\tilde{\nu}_{2}\right|,|s|$ large enough and $|y| e^{s} \leq 4 \varepsilon_{0}$,

$$
\partial_{s} z-\Delta z+\frac{1}{2} y \cdot \nabla z-(1+\sigma) z \leq C\left(z^{2}+e^{s}+\chi_{\varepsilon_{0}}\right),
$$

where $\sigma=C \varepsilon_{0}=\frac{1}{100}$. Now, we consider the cutoff function $\gamma$, we define $Z=z \gamma$ and we obtain for $|s|$ large:

$$
\begin{aligned}
& \partial_{s} Z-\Delta Z+\frac{1}{2} y \cdot \nabla Z-(1+\sigma) Z \\
& \leq C\left(Z^{2}+e^{s}+\chi_{\varepsilon_{0}}\right)-z\left(\partial_{s} \gamma-\Delta \gamma+\frac{y}{2} \cdot \nabla \gamma\right)+2 \nabla \gamma \nabla z .
\end{aligned}
$$

The last terms in this equation are the cutoff terms. Using $z\left(\partial_{s} \gamma-\Delta \gamma+\frac{y}{2} \cdot \nabla \gamma\right)-$ $2 \nabla \gamma \nabla z \leq C \chi_{\varepsilon_{0}}+2 \operatorname{div}(z \nabla \gamma)$, we obtain for $|s|$ large:

$$
\partial_{s} Z-\Delta Z+\frac{1}{2} y \cdot \nabla Z-(1+\sigma) Z \leq C\left(Z^{2}+e^{s}+\chi_{\varepsilon_{0}}\right)-2 \operatorname{div}\left(\left(\left|\tilde{\nu}_{1}\right|+\left|\tilde{\nu}_{2}\right|\right) \nabla \gamma\right),
$$

which is the desired equation in (3.48).

\section{REFERENCES}

[AHV97] D. Andreucci, M. A. Herrero, and J. J. L. Velázquez. Liouville theorems and blow up behaviour in semilinear reaction diffusion systems. Ann. Inst. H. Poincaré Anal. Non Linéaire, 14(1):1-53, 1997. MR1437188 (98e:35088)

[Bal77] J. M. Ball. Remarks on blow-up and nonexistence theorems for nonlinear evolution equations. Quart. J. Math. Oxford Ser. (2), 28(112):473-486, 1977. MR0473484 (57:13150)

[FK92] S. Filippas and R.V. Kohn. Refined asymptotics for the blowup of $u_{t}-\Delta u=u^{p}$. Comm. Pure Appl. Math., 45(7):821-869, 1992. MR1164066 (93g:35066)

[FM95] S. Filippas and F. Merle. Modulation theory for the blowup of vector-valued nonlinear heat equations. J. Differential Equations, 116(1):119-148, 1995. MR1317705 (95m:35087)

[Fuj66] H. Fujita. On the blowing up of solutions of the Cauchy problem for $u_{t}=\Delta u+u^{1+\alpha}$. J. Fac. Sci. Univ. Tokyo Sect. I, 13:109-124 (1966), 1966. MR0214914(35:5761)

[GH96] M. Grayson and R. S. Hamilton. The formation of singularities in the harmonic map heat flow. Comm. Anal. Geom., 4(4):525-546, 1996. MR.1428088 (98g:58034)

[GK85] Y. Giga and R.V. Kohn. Asymptotically self-similar blow-up of semilinear heat equations. Comm. Pure Appl. Math., 38(3):297-319, 1985. MR784476 (86k:35065)

[GMS04] Y. Giga, S. Matsui, and S. Sasayama. Blow up rate for semilinear heat equations with subcritical nonlinearity. Indiana Univ. Math. J., 53(2):483-514, 2004. MR2060042 (2005g:35153)

[GS81a] B. Gidas and J. Spruck. Global and local behavior of positive solutions of nonlinear elliptic equations. Comm. Pure Appl. Math., 34(4):525-598, 1981. MR615628(83f:35045)

[GS81b] B. Gidas and J. Spruck. A priori bounds for positive solutions of nonlinear elliptic equations. Comm. Partial Differential Equations, 6(8):883-901, 1981. MR619749 (82h:35033)

[Ham95] R.S. Hamilton. The formation of singularities in the Ricci flow. In Surveys in differential geometry, Vol. II (Cambridge, MA, 1993), pages 7-136. Int. Press, Cambridge, MA, 1995. MR1375255 (97e:53075)

[HV93] M. A. Herrero and J. J. L. Velázquez. Blow-up behaviour of one-dimensional semilinear parabolic equations. Ann. Inst. H. Poincaré Anal. Non Linéaire, 10(2):131-189, 1993. MR.1220032 (94g:35030)

[Lev73] H. A. Levine. Some nonexistence and instability theorems for solutions of formally parabolic equations of the form $P u_{t}=-A u+F(u)$. Arch. Rational Mech. Anal., 51:371-386, 1973. MR0348216 (50:714) 
[LO96] C. D. Levermore and M. Oliver. The complex Ginzburg-Landau equation as a model problem. In Dynamical systems and probabilistic methods in partial differential equations (Berkeley, CA, 1994), volume 31 of Lectures in Appl. Math., pages 141-190. Amer. Math. Soc., Providence, RI, 1996. MR1363028 (96k:35168)

[MM00] Y. Martel and F. Merle. A Liouville theorem for the critical generalized Kortewegde Vries equation. J. Math. Pures Appl. (9), 79(4):339-425, 2000. MR1753061 (2001i:37102)

[MR04] F. Merle and P. Raphael. On universality of blow-up profile for $L^{2}$ critical nonlinear Schrödinger equation. Invent. Math., 156(3):565-672, 2004. MR2061329 (2006a:35283)

[MR05] F. Merle and P. Raphael. The blow-up dynamic and upper bound on the blow-up rate for critical nonlinear Schrödinger equation. Ann. of Math. (2), 161(1):157-222, 2005. MR2150386 (2006k:35277)

[MZ98a] F. Merle and H. Zaag. Optimal estimates for blowup rate and behavior for nonlinear heat equations. Comm. Pure Appl. Math., 51(2):139-196, 1998. MR1488298 (98k:35107)

[MZ98b] F. Merle and H. Zaag. Refined uniform estimates at blow-up and applications for nonlinear heat equations. Geom. Funct. Anal., 8(6):1043-1085, 1998. MR1664791 (2000c:35106)

[MZ00] F. Merle and H. Zaag. A Liouville theorem for vector-valued nonlinear heat equations and applications. Math. Ann., 316(1):103-137, 2000. MR.1735081(2001d:35084)

[MZ08a] N. Masmoudi and H. Zaag. Blow-up profile for the complex Ginzburg-Landau equation. J. Funct. Anal., 255(7):1613-1666, 2008. MR2442077

[MZ08b] F. Merle and H. Zaag. Openness of the set of non-characteristic points and regularity of the blow-up curve for the $1 \mathrm{D}$ semilinear wave equation. Comm. Math. Phys.,282(1):5586, 2008. MR 2415473

[Nou08] N. Nouaili. A simplified proof of a Liouville theorem for nonnegative solution of a subcritical semilinear heat equations. J. Dynam. Differential Equations, 2008. to appear.

[PKK98] S. Popp, O. Stiller, E. Kuznetsov, and L. Kramer. The cubic complex Ginzburg-Landau equation for a backward bifurcation. Phys. D, 114(1-2):81-107, 1998. MR1612047 (98k:35181)

[PŠ01] P. Plecháč and V. Šverák. On self-similar singular solutions of the complex GinzburgLandau equation. Comm. Pure Appl. Math., 54(10):1215-1242, 2001. MR.1843986 (2002d:35194)

[Vel92] J. J. L. Velázquez. Higher-dimensional blow up for semilinear parabolic equations. Comm. Partial Differential Equations, 17(9-10):1567-1596, 1992. MR 1187622 (93k:35044)

[Vel93] J. J. L. Velázquez. Classification of singularities for blowing up solutions in higher dimensions. Trans. Amer. Math. Soc., 338(1):441-464, 1993. MR.1134760 (93j:35101)

[Wei84] F. B. Weissler. Single point blow-up for a semilinear initial value problem. J. Differential Equations, 55(2):204-224, 1984. MR764124 (86a:35076)

[Zaa98] H. Zaag. Blow-up results for vector-valued nonlinear heat equations with no gradient structure. Ann. Inst. H. Poincaré Anal. Non Linéaire, 15(5):581-622, 1998. MR1643389 (99i:35066)

[Zaa01] H. Zaag. A Liouville theorem and blowup behavior for a vector-valued nonlinear heat equation with no gradient structure. Comm. Pure Appl. Math., 54(1):107-133, 2001. MR:1787109(2001h:35088)

Département de Mathématiques et Applications, École Normale Supérieure, 45 rue D'Ulm 75230, Paris Cedex 05, France

Current address: Université Paris 13, Institut Galilée, Laboratoire Analyse, Géométrie et Applications, CNRS-UMR 7539, 99 avenue J.B. Clément, 93430 Villetaneuse, France

E-mail address: nouaili@math.univ-paris13.fr

Université Paris 13, Institut Galilée, Laboratoire Analyse, Géométrie et Applications, CNRS-UmR 7539, 99 avenue J.B. Clément, 93430 Villetaneuse, France

E-mail address: zaag@math.univ-paris13.fr 